\title{
Equivariant branes and equivariant homological mirror symmetry
}

\author{
Meer Ashwinkumar ${ }^{*}$ and Meng-Chwan Tan ${ }^{\dagger}$ \\ Department of Physics, National University of Singapore, 2 Science Drive 3, 117551 Singapore
}

(Received 10 October 2017; published 13 March 2018)

\begin{abstract}
We describe supersymmetric A-branes and B-branes in open $\mathcal{N}=(2,2)$ dynamically gauged nonlinear sigma models (GNLSM), placing emphasis on toric manifold target spaces. For a subset of toric manifolds, these equivariant branes have a mirror description as branes in gauged Landau-Ginzburg models with neutral matter. We then study correlation functions in the topological A-twisted version of the GNLSM and identify their values with open Hamiltonian Gromov-Witten invariants. Supersymmetry breaking can occur in the A-twisted GNLSM due to nonperturbative open symplectic vortices, and we canonically Becchi-Rouet-Stora-Tyutin quantize the mirror theory to analyze this phenomenon.
\end{abstract}

DOI: 10.1103/PhysRevD.97.066010

\section{INTRODUCTION}

D-branes are crucial to the nonperturbative dynamics of string theory, and their importance has been well understood since Polchinski [1] identified them with black p-brane solutions of supergravity. From the point of view of mathematics, D-branes are essential objects of homological mirror symmetry, first conjectured by Kontsevich [2].

In this paper, we investigate D-branes of dynamically gauged nonlinear sigma models (GNLSMs) with $\mathcal{N}=$ $(2,2)$ supersymmetry, which we shall refer to as equivariant branes. One motivation for this is that GNLSMs with target space $X$ and gauge group $G$ flow in the IR limit to nonlinear sigma models (NLSMs) with target space $X / / G$, and hence, we will obtain new descriptions of D-branes in $\mathcal{N}=(2,2) \quad$ NLSMs, including those with Calabi-Yau targets useful for physical compactifications of string theory. A more mathematically oriented motivation is furnishing an equivariant generalization of homological mirror symmetry. As we shall see, describing equivariant branes will also allow us to define an open version of the mathematical theory of Hamiltonian Gromov-Witten invariants [3-5].

The $\mathcal{N}=(2,2)$ GNLSM governing maps from a closed Riemann surface into a Kähler manifold $X$ with Hamiltonian isometry group $G$ was studied in depth by Baptista [6,7]. In particular, it was shown that the A-twisted GNLSM localizes to the moduli space of symplectic

\footnotetext{
"meerashwinkumar@u.nus.edu

†mctan@nus.edu.sg
}

Published by the American Physical Society under the terms of the Creative Commons Attribution 4.0 International license. Further distribution of this work must maintain attribution to the author(s) and the published article's title, journal citation, and DOI. Funded by SCOAP . vortices, and its correlation functions compute the Hamiltonian Gromov-Witten invariants of $X$. Moreover, for Abelian $G$, Baptista used mirror symmetry (as proven by Hori and Vafa [8]) to describe the quantum equivariant cohomology ring for toric $X$.

Also, D-branes in $\mathcal{N}=(2,2)$ NLSMs on open Riemann surfaces have been studied by Hori, Iqbal, and Vafa $[9,10]$, with the mirrors of these D-branes being identified. We are thus led to attempt an understanding of equivariant branes by combining the insights described above, that is, by analyzing $\mathcal{N}=(2,2)$ GNLSMs on open Riemann surfaces and their mirrors. Since only a subset of the $\mathcal{N}=$ $(2,2)$ supersymmetry can be preserved at the boundaries of these open Riemann surfaces, we are led to two types of equivariant branes, namely, equivariant $\mathrm{A}$-branes and equivariant B-branes.

Equivariant B-branes have been previously studied by Kapustin et al. [11] within the context of topologically B-twisted GNLSMs, although the mirrors of these branes were not elucidated. On the other hand, equivariant A-branes have only been studied for $G=U(1)$ by Setter [12], using the topologically A-twisted nondynamical $U(1)$-GNLSM; in this case, their mirrors were not elucidated either. We shall study both types of equivariant branes and provide the description of their mirrors in a subset of toric target spaces, hence defining equivariant homological mirror symmetry in these contexts. Other proposals for equivariant homological mirror symmetry of equivariant B-branes have appeared in the mathematical literature $[13,14]$. In addition, understanding equivariant A-branes allows us to define open Hamiltonian GromovWitten invariants, which can be understood as integrals over the moduli spaces of open symplectic vortices that describe a map from an open Riemann surface $\Sigma$ to a Kähler and Hamiltonian $G$-manifold $X$, whereby the boundaries of $\Sigma$ correspond to equivariant A-branes in $X$. We note that 
closed Hamiltonian Gromov-Witten invariants have been studied extensively in the mathematical literature [3-5]. However, the open invariants have been largely unexplored, with the exception of the work of Xu [15], which concerns the compactification of the moduli space of open symplectic vortices on the disk for $G=U(1)$, as well as the work of Wang and $\mathrm{Xu}$ [16] on the relationship between open symplectic vortices for $X$ and open world sheet instantons for $X / / G$ (the open quantum Kirwan map).

\section{A. An outline of the paper}

In Sec. II, we introduce the $\mathcal{N}=(2,2)$ supersymmetric dynamically gauged nonlinear sigma model on the infinite strip. In Sec. III, we review the mirror symmetry between Abelian GNLSMs and gauged Landau-Ginzburg (LG) models with neutral matter, and elucidate the explicit reduction of open gauged linear sigma models (GLSMs) to open GNLSMs, making use of the example of $\mathbb{C} P^{N-1}$. In Sec. IV, we study equivariant B-branes, paying particular attention to Abelian equivariant B-branes in toric manifolds $X$, as well as the LG mirrors of these branes when $X$ is Fano. Non-Abelian equivariant B-branes in general $G$ manifolds are also analyzed. In Sec. V, Abelian equivariant A-branes in toric manifolds $X$ are introduced, and their LG mirror description is shown for toric manifolds $X$ with $c_{1}(X) \geq 0$. We also explore non-Abelian equivariant A-branes for general $G$ manifolds. In Sec. VI, we use the data of equivariant A-branes to study open Hamiltonian Gromov-Witten invariants. The open gauged $A$ model is first introduced, together with its bulk and boundary observables. The path integrals of these observables are given by classical integrals over the moduli spaces of open symplectic vortices on a Riemann surface with boundaries, and these integrals are identified with the open Hamiltonian Gromov-Witten invariants. Then, we compute the dimension of these moduli spaces, as well as the related boundary axial $R$ anomaly. For Abelian invariants, we use mirror symmetry to compute the $\hat{Q}_{A}^{2} \neq 0$ anomaly, which implies supersymmetry breaking and indicates an obstruction to integration over the moduli spaces. We shall find the condition whereby the anomaly vanishes and supersymmetry is manifest. Finally, we show how mirror symmetry can be used to compute the Abelian invariants themselves.

The reader who is interested in equivariant B-branes should read Secs. II, III, and IV, whereas the reader who is interested in equivariant A-branes and open Hamiltonian Gromov-Witten invariants should read Secs. II, III, V, and VI. Additional details are contained in the comprehensive version of this paper [17].

\section{THE GAUGED NONLINEAR SIGMA MODEL WITH BOUNDARIES}

We shall take the world sheet $\Sigma$ to be the infinite strip $I \times \mathbb{R}$ (where the interval is $I=[0, \pi]$ ) equipped with a flat Minkowski metric $\eta=\operatorname{diag}(-1,1)$. The main fields of the GNLSM are a connection, $A$, on a principal $G$-bundle $P \rightarrow \Sigma$, and a section

$$
\phi: \Sigma \rightarrow E
$$

of the associated bundle $E:=P \times_{G} X$, where $X$ is a Kähler manifold. Locally on $\Sigma, E$ looks like the product $\Sigma \times X$, which implies that locally the section $\phi$ looks like a map $\phi: \Sigma \rightarrow X$, as one finds in nongauged NLSMs.

The GNLSM action is

$$
S=\frac{1}{2 \pi}\left(S_{\text {matter }}+S_{\text {gauge }}+S_{B}+S_{\theta}\right),
$$

where

$$
\begin{aligned}
& S_{\text {matter }}=\int_{\Sigma} d^{2} x\left(-g_{j \bar{k}} \partial_{\mu}^{A} \phi^{j} \partial^{A \mu} \bar{\phi}^{\bar{k}}+\frac{i}{2} g_{j} \overline{\bar{\psi}} \bar{\psi}_{-}^{\bar{k}}\left(\phi^{*} \stackrel{\leftrightarrow}{\nabla}^{A}\right)_{+} \psi_{-}^{j}+\frac{i}{2} g_{j \bar{k}} \bar{\psi}_{+}^{\bar{k}}\left(\phi^{*} \stackrel{\leftrightarrow}{\nabla}^{A}\right)_{-} \psi_{+}^{j}\right. \\
& -\frac{1}{2} g_{j \bar{k}} \sigma^{a} \tilde{e}_{a}^{j} \bar{\sigma}^{b} \overline{\tilde{e}}_{b}^{\bar{k}}-\frac{1}{2} g_{j \bar{k}} \bar{\sigma}^{a} \tilde{e}_{a}^{j} \sigma^{b} \overline{\tilde{e}}_{b}^{\bar{k}}+i g_{j \bar{k}}\left(\nabla_{l} \tilde{e}_{a}^{j}\right)\left(\sigma^{a} \bar{\psi}_{-}^{\bar{k}} \psi_{+}^{l}+\bar{\sigma}^{a} \bar{\psi}_{+}^{\bar{k}} \psi_{-}^{l}\right) \\
& +g_{j \bar{k}}\left(\bar{\lambda}_{+}^{a} \tilde{e}_{a}^{j} \bar{\psi}_{-}^{\bar{k}}-\bar{\lambda}_{-}^{a} \tilde{e}_{a}^{j} \bar{\psi}_{+}^{\bar{k}}-\lambda_{+}^{a} \overline{\tilde{e}}_{a}^{\bar{k}} \psi_{-}^{j}+\lambda_{-}^{a} \overline{\tilde{e}}_{a}^{\bar{k}} \psi_{+}^{j}\right) \\
& \left.+R_{i \bar{\jmath} k \bar{l}} \psi_{+}^{i} \psi_{-}^{k} \bar{\psi}_{-}^{\bar{\jmath}} \bar{\psi}_{+}^{\bar{l}}+g_{j \bar{k}}\left(F^{j}-\Gamma_{i l}^{j} \psi_{+}^{i} \psi_{-}^{l}\right)\left(\bar{F}^{\bar{k}}-\Gamma_{\bar{m}}^{\bar{k}} \overline{\boldsymbol{\psi}}_{-}^{\bar{m}} \bar{\psi}_{+}^{\bar{n}}\right)\right), \\
& S_{\text {gauge }}=\frac{1}{2 e^{2}} \int_{\Sigma} d^{2} x\left(F_{01}^{a} F_{01 a}-\nabla_{\mu}^{A} \sigma^{a} \nabla^{A \mu} \bar{\sigma}_{a}+\frac{1}{4}[\sigma, \bar{\sigma}]^{a}[\sigma, \bar{\sigma}]_{a}+D^{a} D_{a}-2 e^{2}\left(\phi^{*} \mu_{a}+r_{a}\right) D^{a}\right. \\
& \left.+\frac{i}{2}\left(\bar{\lambda}_{-}\right)_{a} \stackrel{\leftrightarrow}{\nabla}_{+}^{A} \lambda_{-}^{a}+\frac{i}{2}\left(\bar{\lambda}_{+}\right)_{a} \stackrel{\leftrightarrow}{\nabla}_{-}^{A} \lambda_{+}^{a}+i \bar{\lambda}_{-}^{a}\left[\sigma, \lambda_{+}\right]_{a}+i \bar{\lambda}_{+}^{a}\left[\bar{\sigma}, \lambda_{-}\right]_{a}\right), \\
& S_{B}=-\int_{\Sigma} \phi^{*} B+\int_{\partial \Sigma} \phi^{*} C_{a} A^{a}
\end{aligned}
$$


and

$$
S_{\theta}=\int_{\Sigma} d^{2} x\left(\theta, F_{01}\right)
$$

with $d^{2} x=d x^{1} \wedge d x^{0}{ }^{1}$ Here, $F^{j}$ and $D^{a}$ are auxiliary fields. The covariant derivatives are given explicitly as

$$
\begin{aligned}
\partial_{\mu}^{A} \phi^{k} & =\partial_{\mu} \phi^{k}+A_{\mu}^{a} \tilde{e}_{a}^{k}, \\
\left(\phi^{*} \nabla^{A}\right)_{\mu} \psi^{k} & =\partial_{\mu} \psi^{k}+A_{\mu}^{a} \psi^{j} \partial_{j} \tilde{e}_{a}^{k}+\Gamma_{j l}^{k}\left(\partial_{\mu}^{A} \phi^{j}\right) \psi^{l}, \\
\nabla_{\mu}^{A} \sigma^{a} & =\partial_{\mu} \sigma^{a}+\left[A_{\mu}, \sigma\right]^{a}, \\
\nabla_{\mu}^{A} \lambda^{a} & =\partial_{\mu} \lambda^{a}+\left[A_{\mu}, \lambda\right]^{a} .
\end{aligned}
$$

We have also used the notations

$$
\begin{aligned}
& \nabla_{+}=\nabla_{0}+\nabla_{1}, \\
& \nabla_{-}=\nabla_{0}-\nabla_{1},
\end{aligned}
$$

as well as

$$
A \stackrel{\leftrightarrow}{\nabla} B=A \nabla B-\nabla B A .
$$

Additional details regarding the GNLSM action can be found in $[6,17]$. The main difference from the closed GNLSM is the boundary term with a $C$ field, where $C$ is an equivariant map $X \rightarrow \mathfrak{g}^{*}$ that obeys

$$
d C_{a}=l_{\tilde{e}_{a}} B .
$$

This term is required to cancel the nonzero gauge variation of the $B$-field term, leaving the action invariant under the following gauge transformations:

$$
\begin{aligned}
\delta \phi^{k} & =\alpha^{a} \tilde{e}_{a}^{k}, \\
\delta \bar{\phi}^{\bar{k}} & =\alpha^{a} \overline{\tilde{e}}_{a}^{\bar{k}}, \\
\delta \psi_{ \pm}^{k} & =\alpha^{a} \psi_{ \pm}^{i} \partial_{i} \tilde{e}_{a}^{k}, \\
\delta \bar{\psi}_{ \pm}^{\bar{k}} & =\alpha^{a} \bar{\psi}_{ \pm}^{\bar{\imath}} \partial_{\bar{\imath}} \overline{\tilde{e}}_{a}^{\bar{k}}, \\
\delta F^{k} & =\alpha^{a} F^{i} \partial_{i} \tilde{e}_{a}^{k}, \\
\delta \bar{F}^{\bar{k}} & =\alpha^{a} \bar{F}^{\bar{\imath}} \partial_{\bar{\imath}} \overline{\tilde{e}}_{a}^{\bar{k}} ;
\end{aligned}
$$

$$
\begin{aligned}
\delta A_{\mu}^{a} & =\left[\alpha, A_{\mu}\right]^{a}-\partial_{\mu} \alpha^{a}=-\nabla_{\mu}^{A} \alpha^{a}, \\
\delta \sigma^{a} & =[\alpha, \sigma]^{a}, \\
\delta \bar{\sigma}^{a} & =[\alpha, \bar{\sigma}]^{a}, \\
\delta \lambda_{ \pm}^{a} & =\left[\alpha, \lambda_{ \pm}\right]^{a}, \\
\delta \bar{\lambda}_{ \pm}^{a} & =\left[\alpha, \bar{\lambda}_{ \pm}\right]^{a}, \\
\delta D^{a} & =[\alpha, D]^{a} .
\end{aligned}
$$

\footnotetext{
${ }^{1}$ We consider only the case where $G$ is compact, in order to ensure positive definiteness of the gauge multiplet kinetic terms.
}

For a closed world sheet, the action (2.2) would be invariant under the following $\mathcal{N}=(2,2)$ supersymmetry transformations:

$$
\begin{aligned}
\delta \phi^{k}= & \left(\epsilon_{+} \psi_{-}^{k}-\epsilon_{-} \psi_{+}^{k}\right), \\
\delta \bar{\phi}^{\bar{k}}= & -\left(\bar{\epsilon}_{+} \bar{\psi}_{-}^{\bar{k}}-\bar{\epsilon}_{-} \bar{\psi}_{+}^{\bar{k}}\right), \\
\delta \psi_{+}^{k}= & i \bar{\epsilon}_{-}\left(\partial_{0}^{A}+\partial_{1}^{A}\right) \phi^{k}+\epsilon_{+} F^{k}+i \bar{\epsilon}_{+} \bar{\sigma}^{a} \tilde{e}_{a}^{k}, \\
\delta \bar{\psi}_{+}^{\bar{k}}= & -i \epsilon_{-}\left(\partial_{0}^{A}+\partial_{1}^{A}\right) \bar{\phi}^{\bar{k}}+\bar{\epsilon}_{+} \bar{F}^{\bar{k}}-i \epsilon_{+} \sigma^{a} \overline{\tilde{e}}_{a}^{\bar{k}}, \\
\delta \psi_{-}^{k}= & -i \bar{\epsilon}_{+}\left(\partial_{0}^{A}-\partial_{1}^{A}\right) \phi^{k}+\epsilon_{-} F^{k}-i \bar{\epsilon}_{-} \sigma^{a} \tilde{e}_{a}^{k}, \\
\delta \bar{\psi}_{-}^{\bar{k}}= & i \epsilon_{+}\left(\partial_{0}^{A}-\partial_{1}^{A}\right) \bar{\phi}^{\bar{k}}+\bar{\epsilon}_{-} \bar{F}^{\bar{k}}+i \epsilon_{-} \bar{\sigma}^{a} \overline{\tilde{e}}_{a}^{\bar{k}}, \\
\delta F^{k}= & -i \bar{\epsilon}_{+}\left(\partial_{-} \psi_{+}^{k}+A_{-}^{a}\left(\partial_{j} \tilde{e}_{a}^{k}\right) \psi_{+}^{j}\right) \\
& -i \bar{\epsilon}_{-}\left(\partial_{+} \psi_{-}^{k}+A_{+}^{a}\left(\partial_{j} \tilde{e}_{a}^{k}\right) \psi_{-}^{j}\right)+\bar{\epsilon}_{-} \bar{\lambda}_{+}^{a} \tilde{e}_{a}^{k}-\bar{\epsilon}_{+} \bar{\lambda}_{-}^{a} \tilde{e}_{a}^{k} \\
& -i \bar{\epsilon}_{+} \bar{\sigma}^{a}\left(\partial_{j} \tilde{e}_{a}^{k}\right) \psi_{-}^{j}-i \bar{\epsilon}_{-} \sigma^{a}\left(\partial_{j} \tilde{e}_{a}^{k}\right) \psi_{+}^{j}, \\
\delta \bar{F}^{\bar{k}}= & -i \epsilon_{+}\left(\partial_{-} \bar{\psi}_{+}^{\bar{k}}+A_{-}^{a}\left(\partial_{\bar{\jmath}} \overline{\tilde{e}}_{a}^{\bar{k}}\right) \bar{\psi}_{+}^{\bar{j}}\right) \\
& -i \epsilon_{-}\left(\partial_{+} \bar{\psi}_{-}^{\bar{k}}+A_{+}^{a}\left(\partial_{\bar{\jmath}} \overline{\tilde{e}}_{a}^{\bar{k}}\right) \bar{\psi}_{-}^{\bar{j}}\right)-\epsilon_{-} \lambda_{+}^{a} \overline{\tilde{e}}_{a}^{\bar{k}}+\epsilon_{+} \lambda_{-}^{a} \overline{\tilde{e}}_{a}^{\bar{k}} \\
& -i \epsilon_{+} \sigma^{a}\left(\partial_{\bar{\jmath}} \overline{\tilde{e}}_{a}^{\bar{k}}\right) \bar{\psi}_{-}^{\bar{j}}-i \epsilon_{-} \bar{\sigma}^{a}\left(\partial_{\bar{\jmath}} \overline{\tilde{e}}_{a}^{\bar{k}}\right) \bar{\psi}_{+}^{\bar{j}}, \quad(2.13
\end{aligned}
$$

$$
\begin{aligned}
\delta A_{+}^{a}= & i \epsilon_{+} \bar{\lambda}_{+}^{a}+i \bar{\epsilon}_{+} \lambda_{+}^{a}, \\
\delta A_{-}^{a}= & i \epsilon_{-} \bar{\lambda}_{-}^{a}+i \bar{\epsilon}_{-} \lambda_{-}^{a}, \\
\delta \sigma^{a}= & -i \bar{\epsilon}_{+} \lambda_{-}^{a}-i \epsilon_{-} \bar{\lambda}_{+}^{a}, \\
\delta \bar{\sigma}^{a}= & -i \epsilon_{+} \bar{\lambda}_{-}^{a}-i \bar{\epsilon}_{-} \lambda_{+}^{a}, \\
\delta \lambda_{+}^{a}= & \epsilon_{-}\left(\nabla_{+}^{A} \bar{\sigma}^{a}\right)+\epsilon_{+}\left(-F_{01}^{a}+\frac{1}{2}[\sigma, \bar{\sigma}]^{a}+i D^{a}\right), \\
\delta \bar{\lambda}_{+}^{a}= & \bar{\epsilon}_{-}\left(\nabla_{+}^{A} \sigma^{a}\right)+\bar{\epsilon}_{+}\left(-F_{01}^{a}-\frac{1}{2}[\sigma, \bar{\sigma}]^{a}-i D^{a}\right), \\
\delta \lambda_{-}^{a}= & \epsilon_{+}\left(\nabla_{-}^{A} \sigma^{a}\right)+\epsilon_{-}\left(F_{01}^{a}-\frac{1}{2}[\sigma, \bar{\sigma}]^{a}+i D^{a}\right), \\
\delta \bar{\lambda}_{-}^{a}= & \bar{\epsilon}_{+}\left(\nabla_{-}^{A} \bar{\sigma}^{a}\right)+\bar{\epsilon}_{-}\left(F_{01}^{a}+\frac{1}{2}[\sigma, \bar{\sigma}]^{a}-i D^{a}\right), \\
\delta D^{a}= & \frac{1}{2}\left(-\bar{\epsilon}_{+}\left(\nabla_{-}^{A} \lambda_{+}^{a}\right)-\bar{\epsilon}_{-}\left(\nabla_{+}^{A} \lambda_{-}^{a}\right)+\epsilon_{+}\left(\nabla_{-}^{A} \bar{\lambda}_{+}^{a}\right)\right. \\
& +\epsilon_{-}\left(\nabla_{+}^{A} \bar{\lambda}_{-}^{a}\right)+\epsilon_{+}\left[\sigma, \bar{\lambda}_{-}\right]^{a}+\epsilon_{-}\left[\bar{\sigma}, \bar{\lambda}_{+}\right]^{a} \\
& \left.-\bar{\epsilon}_{+}\left[\bar{\sigma}_{-} \lambda_{-}\right]^{a}-\bar{\epsilon}_{-}\left[\sigma, \lambda_{+}\right]^{a}\right),
\end{aligned}
$$

where $A_{ \pm}=A_{0} \pm A_{1}, \partial_{ \pm}=\partial_{0} \pm \partial_{1}$, and

$$
\delta=\epsilon_{+} Q_{-}-\epsilon_{-} Q_{+}-\bar{\epsilon}_{+} \bar{Q}_{-}+\bar{\epsilon}_{-} \bar{Q}_{+}
$$

in terms of the supercharges of the $\mathcal{N}=(2,2)$ supersymmetry algebra. On the other hand, since our theory is defined on $I \times \mathbb{R}$, supersymmetry is not preserved at the boundaries, and we have 


$$
\begin{aligned}
& \delta S_{\text {matter }}=\frac{1}{2 \pi} \int_{\partial \Sigma} d x^{0}\left\{-\epsilon_{+}\left(\frac{1}{2} g_{i \bar{j}}\left(\partial_{0}^{A}+\partial_{1}^{A}\right) \bar{\phi}^{\bar{\jmath}} \psi_{-}^{i}+\frac{1}{2} g_{i \bar{j}} \sigma^{a} \overline{\tilde{e}}_{a}^{\bar{J}} \psi_{+}^{i}-\frac{i}{2} g_{i \bar{\jmath}} F^{i} \bar{\psi}_{+}^{\bar{\jmath}}+\frac{i}{2} g_{i \bar{j}} \Gamma_{j l}^{i} \psi_{+}^{j} \psi_{-}^{l} \bar{\psi}_{+}^{\bar{j}}\right)\right. \\
& +\bar{\epsilon}_{+}\left(\frac{1}{2} g_{i \bar{\jmath}}\left(\partial_{0}^{A}+\partial_{1}^{A}\right) \phi^{i} \bar{\psi}_{-}^{\bar{\jmath}}+\frac{1}{2} g_{i \bar{\jmath}} \bar{\sigma}^{a} \tilde{e}_{a}^{i} \bar{\psi}_{+}^{\bar{\jmath}}+\frac{i}{2} g_{i \bar{\jmath}} \bar{F}^{\bar{j}} \psi_{+}^{i}-\frac{i}{2} g_{i \bar{\jmath}} \Gamma_{\bar{l}}^{\bar{\jmath}} \bar{\psi}_{-}^{\bar{\imath}} \bar{\psi}_{+}^{\bar{l}} \psi_{+}^{i}\right) \\
& -\epsilon_{-}\left(\frac{1}{2} g_{i \bar{\jmath}}\left(\partial_{0}^{A}-\partial_{1}^{A}\right) \bar{\phi}^{\bar{j}} \psi_{+}^{i}+\frac{1}{2} g_{i \bar{\jmath}} \bar{\sigma}^{a} \overline{\tilde{e}}_{a}^{\bar{J}} \psi_{-}^{i}+\frac{i}{2} g_{i \bar{j}} F^{i} \bar{\psi}_{-}^{\bar{\jmath}}-\frac{i}{2} g_{i \bar{\jmath}} \Gamma_{j l}^{i} \psi_{+}^{j} \psi_{-}^{l} \bar{\psi}_{-}^{\bar{\jmath}}\right) \\
& \left.+\bar{\epsilon}_{-}\left(\frac{1}{2} g_{i \bar{\jmath}}\left(\partial_{0}^{A}-\partial_{1}^{A}\right) \phi^{i} \bar{\psi}_{+}^{\bar{\jmath}}+\frac{1}{2} g_{i \bar{\jmath}} \sigma^{a} \tilde{e}_{a}^{i} \bar{\psi}_{-}^{\bar{\jmath}}-\frac{i}{2} g_{i \bar{\jmath}} \bar{F}^{\bar{j}} \psi_{-}^{i}+\frac{i}{2} g_{i \bar{\jmath}} \Gamma_{\bar{l}}^{\bar{\jmath}} \bar{\psi}_{-}^{\bar{\imath}} \bar{\psi}_{+}^{\bar{l}} \psi_{-}^{i}\right)\right\}, \\
& \delta\left(S_{\text {gauge }}+S_{r}+S_{\theta}\right)=\frac{1}{2 \pi} \frac{1}{2 e^{2}} \int_{\partial \Sigma} d x^{0}\left\{\epsilon_{+}\left(\frac{i}{2} \bar{\lambda}_{-a}\left(\nabla_{1}^{A}+\nabla_{0}^{A}\right) \sigma^{a}-\bar{\lambda}_{+a}\left(\frac{i}{2} F_{01}^{a}+\frac{i}{4}[\sigma, \bar{\sigma}]^{a}+\frac{D^{a}}{2}\right)+e^{2}\left(\phi^{*} \mu_{a}+r_{a}-i \theta_{a}\right) \bar{\lambda}_{+}^{a}\right)\right. \\
& +\bar{\epsilon}_{+}\left(\frac{i}{2} \lambda_{-a}\left(\nabla_{1}^{A}+\nabla_{0}^{A}\right) \bar{\sigma}^{a}-\lambda_{+a}\left(\frac{i}{2} F_{01}^{a}-\frac{i}{4}[\sigma, \bar{\sigma}]^{a}-\frac{D^{a}}{2}\right)-e^{2}\left(\phi^{*} \mu_{a}+r_{a}+i \theta_{a}\right) \lambda_{+}^{a}\right) \\
& +\epsilon_{-}\left(\frac{i}{2} \bar{\lambda}_{+a}\left(\nabla_{1}^{A}-\nabla_{0}^{A}\right) \bar{\sigma}^{a}-\bar{\lambda}_{-a}\left(\frac{i}{2} F_{01}^{a}+\frac{i}{4}[\sigma, \bar{\sigma}]^{a}-\frac{D^{a}}{2}\right)-e^{2}\left(\phi^{*} \mu_{a}+r_{a}+i \theta_{a}\right) \bar{\lambda}_{-}^{a}\right) \\
& \left.+\bar{\epsilon}_{-}\left(\frac{i}{2} \lambda_{+a}\left(\nabla_{1}^{A}-\nabla_{0}^{A}\right) \sigma^{a}-\lambda_{-a}\left(\frac{i}{2} F_{01}^{a}-\frac{i}{4}[\sigma, \bar{\sigma}]^{a}+\frac{D^{a}}{2}\right)+e^{2}\left(\phi^{*} \mu_{a}+r_{a}-i \theta_{a}\right) \lambda_{-}^{a}\right)\right\}, \\
& \delta S_{B}=\frac{1}{2 \pi} \int_{\partial \Sigma} d x^{0}\left\{\left(B_{i j} \partial_{0}^{A} \phi^{i}+B_{\bar{l} j} \partial_{0}^{A} \bar{\phi}^{\bar{\imath}}\right)\left(\epsilon_{+} \psi_{-}^{j}-\epsilon_{-} \psi_{+}^{j}\right)+\left(B_{i \bar{J}} \partial_{0}^{A} \phi^{i}+B_{\bar{\imath} \bar{j}} \partial_{0}^{A} \bar{\phi}^{\bar{\imath}}\right)\left(-\bar{\epsilon}_{+} \bar{\psi}_{-}^{\bar{\jmath}}+\bar{\epsilon}_{-} \bar{\psi}_{+}^{\bar{\jmath}}\right)\right. \\
& \left.+\frac{i}{2}\left(\epsilon_{+} \bar{\lambda}_{+}^{a}+\bar{\epsilon}_{+} \lambda_{+}^{a}+\epsilon_{-} \bar{\lambda}_{-}^{a}+\bar{\epsilon}_{-} \lambda_{-}^{a}\right) \phi^{*} C_{a}\right\} .
\end{aligned}
$$

To restore supersymmetry at the boundaries, we need to choose an appropriate set of boundary conditions on the fields, and these conditions must themselves be supersymmetric. In fact, only certain $\mathcal{N}=2$ subsets of the four supersymmetries can be restored (A-type or $B$-type supersymmetry). Furthermore, we can include compatible boundary interactions. These boundary conditions/interactions shall correspond to equivariant generalizations of D-branes. Note that one can further generalize the action (2.2) by considering quiver GNLSMs, i.e., with gauge group $G_{1} \times G_{2} \times G_{3} \ldots$, each factor $G_{i}$ having its own coupling constant, $e_{i}$. In Secs. IV and $\mathrm{V}$, we shall focus on finding boundary conditions/ interactions for quiver Abelian GNLSMs on toric manifolds.

\section{GNLSMS FROM GLSMS AND MIRROR SYMMETRY}

It is well known that $\mathcal{N}=(2,2)$ NLSMs with target spaces being toric manifolds can be obtained in the IR limit of $\mathcal{N}=(2,2)$ quiver Abelian GLSMs [18]. Hori and
Vafa [8] made use of this to prove the mirror symmetry of manifolds with non-negative first Chern class in terms of Landau-Ginzburg theories. This proof of mirror symmetry was later applied to world sheets with boundaries, whereby the Landau-Ginzburg mirrors of B-branes [9] and A-branes [10] were found.

As shown by Baptista [7], it is also possible to obtain quiver Abelian GNLSMs on closed world sheets with toric target spaces by taking a different limit of quiver Abelian GLSMs. Moreover, Baptista found the mirror LandauGinzburg theories of these GNLSMs. This then suggests a natural generalization of Baptista's proof to world sheets with boundaries, in order to find equivariant A-branes and B-branes in Abelian GNLSMs, as well as the LandauGinzburg mirrors of these branes. We shall pursue this line of thought in Secs. IV and V.

Before doing so, let us briefly review Baptista's idea for closed world sheets. In superfield language, the action of a $U(1)^{N}$ GLSM with target space $\mathbb{C}^{N}$ is

$$
\begin{aligned}
S_{\mathrm{GLSM}}= & \frac{1}{2 \pi} \int d^{2} x \int d^{4} \theta\left\{\sum_{j=1}^{N} \bar{\Phi}_{j}\left(e^{\hat{Q}_{j}^{b} \hat{V}_{b}+\tilde{Q}_{j}^{c} \tilde{v}_{c}}\right) \Phi_{j}-\sum_{b=1}^{N-k}\left(\frac{1}{2 \hat{e}_{b}^{2}} \overline{\hat{\Sigma}}_{b} \hat{\Sigma}_{b}\right)-\sum_{c=1}^{k}\left(\frac{1}{2 \tilde{e}_{c}^{2}} \overline{\tilde{\Sigma}}_{c} \tilde{\Sigma}_{c}\right)\right\} \\
& +\frac{1}{2 \pi} \frac{1}{2} \int d^{2} x\left(\int d^{2} \tilde{\theta}\left(-\hat{t}^{b} \hat{\Sigma}_{b}-\tilde{t}^{c} \tilde{\Sigma}_{c}\right)+\text { c.c. }\right)
\end{aligned}
$$


where $U(1)^{N}=U(1)^{(N-k)} \times U(1)^{k}$, with the indices $b=1, \ldots, N-k$ and $c=1, \ldots, k$. The mirror of this theory is the following Landau-Ginzburg sigma model with twisted chiral superfields $Y^{i}$ (whose imaginary parts are periodic, with period $2 \pi$ ) and action

$$
\begin{aligned}
S_{\text {dual }}= & \frac{1}{2 \pi} \int d^{2} x \int d^{4} \theta\left\{-\frac{1}{2} \sum_{j=1}^{N}\left(Y^{j}+\bar{Y}^{j}\right) \log \left(Y^{j}+\bar{Y}^{j}\right)-\sum_{b=1}^{N-k}\left(\frac{1}{2 \hat{e}_{b}^{2}} \overline{\hat{\Sigma}}_{b} \hat{\Sigma}_{b}\right)-\sum_{c=1}^{k}\left(\frac{1}{2 \tilde{e}_{c}^{2}} \overline{\tilde{\Sigma}}_{c} \tilde{\Sigma}_{c}\right)\right\} \\
& +\frac{1}{2 \pi} \frac{1}{2} \int d^{2} x\left\{\int d^{2} \tilde{\theta}\left(\left(\hat{Q}_{j}^{b} Y^{j}-\hat{t}^{b}\right) \hat{\Sigma}_{b}+\left(\tilde{Q}_{j}^{c} Y^{j}-\tilde{t}^{c}\right) \tilde{\Sigma}_{c}+\sum_{j=1}^{N} e^{-Y^{j}}\right)+\text { c.c. }\right\} .
\end{aligned}
$$

Taking quantum effects into account, the target space for this Landau-Ginzburg sigma model is the algebraic torus $\left(\mathbb{C}^{\times}\right)^{N}[8]$. In deriving the duality between the two mirror theories, it can be shown that

$$
Y^{j}+\bar{Y}^{j}=2 \bar{\Phi}_{j} e^{\hat{Q}_{j}^{b} \hat{V}_{b}+\tilde{Q}_{j}^{c} \tilde{V}_{c}} \Phi_{j}
$$

which is an explicit relationship between the fields of the mirror theories.

To obtain a GNLSM from (3.1), we take the limit where $\hat{e}_{b} \rightarrow \infty$. The $\hat{\Sigma}_{b}$ kinetic terms vanishes, and the remaining fields belonging to $\hat{V}_{b}$ become auxiliary fields and are integrated out. The resulting sigma model has $\mathbb{C}^{N} / / U(1)^{N-k}$ as target space, but is still gauged, since the vector superfields $\tilde{V}_{c}$ are still present in the action. However, note that to obtain a Kähler target space with $k$ complex dimensions, the Fayet-Iliopoulos (FI) parameters $\hat{r}_{b}$ must be within a Kähler cone. In this way, we obtain the $\mathcal{N}=(2,2) U(1)^{k}$-GNLSM with Kähler $\mathbb{C}^{N} / / U(1)^{N-k}$ target space.

Taking the same limit $\hat{e}_{b} \rightarrow \infty$ in the dual LandauGinzburg sigma model (3.2) makes the $\hat{\Sigma}_{b}$ kinetic terms vanish. $\hat{\Sigma}_{b}$ is then an auxiliary superfield, which we can integrate out to impose the constraints

$$
\hat{Q}_{j}^{b} Y^{j}-\hat{t}^{b}=0
$$

These constraints have the solution

$$
Y^{j}=\hat{s}^{j}+\sum_{c=1}^{k} v_{c}^{j} \Theta^{c}
$$

where $\Theta^{1}, \ldots, \Theta^{k}$ are new twisted chiral fields, the complex constants $\hat{s}^{1}, \ldots, \hat{s}^{N} \in \mathbb{C}$ are any solution of the algebraic relation $\hat{Q}_{j}^{b} \hat{s}^{j}=\hat{t}^{b}$, and $v_{c}^{j}$ are $N$ primitive vectors $v^{1}, \ldots, v^{N} \in \mathbb{Z}^{k}$ [which generate the regular fan associated with $\left.\mathbb{C}^{N} / / U(1)^{N-k}\right]$ that span $\mathbb{Z}^{k}$ and satisfy $\sum_{j}^{N} \hat{Q}_{j}^{b} v^{j}=0$. Thus, the $\hat{e}_{b} \rightarrow \infty$ limit gives the following $U(1)^{k}$-gauged Landau-Ginzburg sigma model with neutral matter and with target $\left(\mathbb{C}^{\times}\right)^{k}$

$$
\begin{aligned}
\hat{S}_{\text {dual }}= & \frac{1}{2 \pi} \int d^{2} x \int d^{4} \theta\left[-\frac{1}{2} \sum_{j=1}^{N}\left(\hat{s}^{j}+\overline{\hat{s}}^{j}+\left\langle v^{j}, \Theta+\bar{\Theta}\right\rangle\right) \log \left(\hat{s}^{j}+\overline{\hat{s}}^{j}+\left\langle v^{j}, \Theta+\bar{\Theta}\right\rangle\right)-\sum_{c=1}^{k}\left(\frac{1}{2 \tilde{e}_{c}^{2}} \overline{\tilde{\Sigma}}_{c} \tilde{\Sigma}_{c}\right)\right] \\
& +\frac{1}{2 \pi} \frac{1}{2} \int d^{2} x\left\{\int d^{2} \tilde{\theta}\left(\left\langle\tilde{\Sigma}, \tilde{Q}_{j}\right\rangle\left(\left\langle v^{j}, \Theta\right\rangle+\hat{s}^{j}\right)-\langle\tilde{\Sigma}, \tilde{t}\rangle+\sum_{j=1}^{N} e^{-\left\langle v^{j}, \Theta\right\rangle-\hat{s}^{j}}\right)+\text { c.c. }\right\},
\end{aligned}
$$

where $\langle\cdot, \cdot\rangle$ is the canonical inner product on $\mathbb{R}^{k}$. This gauged Landau-Ginzburg theory has the holomorphic twisted superpotential

$\tilde{W}(\Theta, \tilde{\Sigma})=\left\langle\tilde{\Sigma}, \tilde{Q}_{j}\right\rangle\left(\left\langle v^{j}, \Theta\right\rangle+\hat{s}^{j}\right)-\langle\tilde{\Sigma}, \tilde{t}\rangle+\sum_{j=1}^{N} e^{-\left\langle v^{j}, \Theta\right\rangle-\hat{s}^{j}}$.

Baptista's technique of obtaining GNLSMs from GLSMs is an extremely powerful one, as it allows us to obtain multiple GNLSMs from a single GLSM, by choosing which coupling constants we wish to send to infinity. This implies the equivalence of several GNLSMs with different Kähler target manifolds, as well as the equivalence of equivariant branes contained in these manifolds. These branes will be the main objective of our study in the following sections. Furthermore, once a particular GNLSM is obtained, even its gauge group can be modified, by demoting some of its $U(1)$ gauge symmetries to global symmetries. These points shall be useful to keep in mind when reading the following sections, where we attempt to study Abelian equivariant branes in as much generality as possible. 


\section{A. Explicit reduction of GLSMs to GNLSMs in the case with boundaries}

We have only discussed the method of obtaining GNLSMs from GLSMs in superfield language for closed world sheets thus far. We would now like to understand this reduction method in component form for an open world sheet, so that we may eventually find explicit boundary actions and boundary conditions in GNLSMs. The $U(1)^{N}=U(1)^{N-k} \times U(1)^{k}$ GLSM action with boundaries is given explicitly as ${ }^{2}$

$$
\begin{aligned}
S_{\mathrm{GLSM}}= & \frac{1}{2 \pi} \sum_{i}^{N} \int d^{2} x\left\{-D_{\mu} \bar{\phi}_{i} D^{\mu} \phi_{i}+\frac{i}{2} \bar{\psi}_{-i}\left(\stackrel{\leftrightarrow}{D}_{0}+\stackrel{\leftrightarrow}{D}_{1}\right) \psi_{-i}+\frac{i}{2} \bar{\psi}_{+i}\left(\stackrel{\leftrightarrow}{D}_{0}-\stackrel{\leftrightarrow}{D}_{1}\right) \psi_{+i}\right. \\
& -\left(\sum_{b}^{N-k} \hat{Q}_{i b} \hat{\sigma}_{b}+\sum_{c}^{k} \tilde{Q}_{i c} \tilde{\sigma}_{c}\right)\left(\sum_{b}^{N-k} \hat{Q}_{i b} \overline{\hat{\sigma}}_{b}+\sum_{c}^{k} \tilde{Q}_{i c} \overline{\tilde{\sigma}}_{c}\right) \bar{\phi}_{i} \phi_{i} \\
& -\sum_{b}^{N-k} \hat{Q}_{i b}\left(\overline{\hat{\sigma}}_{b} \bar{\psi}_{+i} \psi_{-i}+\hat{\sigma}_{b} \bar{\psi}_{-i} \psi_{+i}\right)-\sum_{c}^{k} \tilde{Q}_{i c}\left(\overline{\tilde{\sigma}}_{c} \bar{\psi}_{+i} \psi_{-i}+\tilde{\sigma}_{c} \bar{\psi}_{-i} \psi_{+i}\right) \\
& -\sum_{b}^{N-k} i \hat{Q}_{i b} \phi_{i}\left(\overline{\hat{\lambda}}_{-b} \bar{\psi}_{+i}-\overline{\hat{\lambda}}_{+b} \bar{\psi}_{-i}\right)-\sum_{c}^{k} i \tilde{Q}_{i c} \phi_{i}\left(\overline{\tilde{\lambda}}_{-c} \bar{\psi}_{+i}-\overline{\tilde{\lambda}}_{+c} \bar{\psi}_{-i}\right) \\
& \left.-\sum_{b}^{N-k} i \hat{Q}_{i b} \bar{\phi}_{i}\left(\psi \psi_{-i} \hat{\lambda}_{+b}-\psi_{+i} \lambda_{-b}\right)-\sum_{c}^{k} i \tilde{Q}_{i c} \bar{\phi}_{i}\left(\psi_{-i} \tilde{\lambda}_{+c}-\psi_{+i} \lambda_{-c}\right)+\left|F_{i}\right|^{2}\right\} \\
& +\frac{1}{2 \pi} \int d^{2} x\left\{\sum_{b}^{N-k}\left(\hat{D}_{b}\left(\sum_{i}^{N} \hat{Q}_{i b} \bar{\phi}_{i} \phi_{i}-\hat{r}_{b}\right)+\hat{\theta}_{b} \hat{F}_{01 b}\right)+\sum_{c}^{k}\left(\tilde{D}_{c}\left(\sum_{i}^{N} \tilde{Q}_{i c} \bar{\phi}_{i} \phi_{i}-\tilde{r}_{c}\right)+\sum_{c}^{k} \tilde{\theta}_{c} \tilde{F}_{01 c}\right)\right\} \\
& +\frac{1}{2 \pi} \sum_{b}^{N-k} \frac{1}{2 \hat{e}_{b}^{2}} \int d^{2} x\left\{\left(\hat{F}_{01 b}\right)^{2}-\partial_{\mu} \hat{\sigma}_{b} \partial^{\mu} \overline{\hat{\sigma}}_{b}+\left(\hat{D}_{b}\right)^{2}+\frac{i}{2} \overline{\hat{\lambda}}_{+b}\left(\stackrel{\leftrightarrow}{\partial}_{0}-\stackrel{\leftrightarrow}{\partial}_{1}\right) \hat{\lambda}_{+b}+\frac{i}{2} \overline{\hat{\lambda}}_{-b}\left(\stackrel{\leftrightarrow}{\partial}_{0}+\stackrel{\leftrightarrow}{\partial}_{1}\right) \hat{\lambda}_{-b}\right\} \\
& \left.+\frac{1}{2 \pi} \sum_{c}^{k} \frac{1}{2 \tilde{e}_{c}^{2}} \int d^{2} x\left\{\left(\tilde{F}_{01 c}\right)^{2}-\partial_{\mu} \tilde{\sigma}_{c} \partial^{\mu} \overline{\tilde{\sigma}}_{c}+\left(\tilde{D}_{c}\right)^{2}+\frac{i}{2} \overline{\tilde{\lambda}}_{+c}\left(\stackrel{\leftrightarrow}{\partial}_{0}-\stackrel{\leftrightarrow}{\partial}_{1}\right) \tilde{\lambda}_{+c}+\frac{i}{2} \overline{\tilde{\lambda}}_{-c} \stackrel{\leftrightarrow}{\partial}_{0}+\stackrel{\leftrightarrow}{\partial}_{1}\right) \tilde{\lambda}_{-c}\right\},
\end{aligned}
$$

where the covariant derivatives are

$$
\begin{aligned}
D_{\mu} \phi_{i} & =\left(\partial_{\mu}+i \sum_{b}^{N-k} \hat{Q}_{i b} \hat{A}_{\mu b}+i \sum_{c}^{k} \tilde{Q}_{i c} \tilde{A}_{\mu c}\right) \phi_{i}, \\
D_{\mu} \psi_{ \pm i} & =\left(\partial_{\mu}+i \sum_{b}^{N-k} \hat{Q}_{i b} \hat{A}_{\mu b}+i \sum_{c}^{k} \tilde{Q}_{i c} \tilde{A}_{\mu c}\right) \psi_{ \pm i} .
\end{aligned}
$$

We shall now take the $\hat{e}_{b} \rightarrow \infty$ limit in (3.8), whereby the vector multiplet kinetic terms as well as the term $\left(\hat{D}_{b}\right)^{2}$ vanish. This means that all the components of the vector superfields $\hat{V}_{b}=\left\{\hat{A}_{\mu b}, \hat{\sigma}_{b}, \hat{\lambda}_{b}, \hat{D}_{b}\right\}$ become auxiliary.

Consequently, the equations of motion of $\hat{A}_{\mu b}$ and $\hat{\sigma}_{b}$ give the following constraints on themselves ${ }^{3}$ :

$$
\begin{aligned}
& \sum_{i}^{N} \hat{Q}_{i b}\left[i\left(\bar{\phi}_{i} D_{0} \phi_{i}-\phi_{i} D_{0} \bar{\phi}_{i}\right)+\bar{\psi}_{-i} \psi_{-i}+\bar{\psi}_{+i} \psi_{+i}\right]=0, \\
& \sum_{i}^{N} \hat{Q}_{i b}\left[i\left(\bar{\phi}_{i} D_{1} \phi_{i}-\phi_{i} D_{1} \bar{\phi}_{i}\right)-\bar{\psi}_{-i} \psi_{-i}+\bar{\psi}_{+i} \psi_{+i}\right]=0,
\end{aligned}
$$

\footnotetext{
${ }^{2}$ It is important to keep in mind that the superfield action (3.1) is only equal to this action upon integration by parts, which give rise to boundary terms. However, we shall be concerned with this action (which has the standard kinetic terms), as well as the GNLSMs we can obtain from it, and their mirrors.

${ }^{3}$ To be precise, the equation of motion for $\hat{A}_{0 b}$ [which is the first equation in (3.10)] will be modified by a boundary term proportional to $\hat{\theta}_{b}$, unless appropriate boundary conditions and/or boundary terms are used. We shall assume that this is the case for now, and in the following sections we will study boundary actions whereby the $\hat{A}_{0 b}$ equation of motion in (3.10) is precise both in the bulk and at the boundaries.
} 


$$
\begin{aligned}
& \sum_{i}^{N}\left[-\hat{Q}_{i b}\left(\sum_{b}^{N-k} \hat{Q}_{i d} \hat{\sigma}_{d}+\sum_{c}^{k} \tilde{Q}_{i c} \tilde{\sigma}_{c}\right) \phi_{i} \bar{\phi}_{i}-\hat{Q}_{i b} \bar{\psi}_{+i} \psi_{-i}\right]=0, \\
& \sum_{i}^{N}\left[-\hat{Q}_{i b}\left(\sum_{d}^{N-k} \hat{Q}_{i d} \overline{\hat{\sigma}}_{d}+\sum_{c}^{k} \tilde{Q}_{i c} \overline{\tilde{\sigma}}_{c}\right) \phi_{i} \bar{\phi}_{i}-\hat{Q}_{i b} \bar{\psi}_{-i} \psi_{+i}\right]=0,
\end{aligned}
$$

while integrating out the gauginos $\left(\hat{\lambda}_{b}\right)$ gives the constraints

$$
\sum_{i}^{N} \hat{Q}_{i b} \bar{\phi}_{i} \psi_{ \pm i}=0,
$$

and finally, integrating out $\hat{D}_{b}$ gives

$$
\sum_{i}^{N} \hat{Q}_{i b} \bar{\phi}_{i} \phi_{i}-\hat{r}_{b}=0,
$$

for $b=1, \ldots, N-k^{4}$

To derive the explicit action for the $U(1)^{k}$-GNLSM with a Kähler target space $\mathbb{C}^{N} / / U(1)^{N-k}$, we need to set the FI parameters $\hat{r}_{b}$ to be in a particular Kähler cone, before taking the $\hat{e}_{b} \rightarrow \infty$ limit. Next, we need to find parametrizations for the scalar fields $\phi_{i}$ that satisfy (3.13), as well as parametrizations for $\psi_{ \pm}$that satisfy (3.12). Then, $\hat{A}_{\mu b}$ and $\hat{\sigma}_{b}$ must be integrated out of the action using (3.10) and (3.11). Finally, we need to replace the matter auxiliary field term

$$
\sum_{i}^{N}\left|F_{i}\right|^{2}
$$

with the matter auxiliary field term of the GNLSM in (2.3).

This procedure is simplest for the case of $N-k=1$, where (3.10) and (3.11) reduce to

$$
\begin{aligned}
& \hat{A}_{0}=\frac{\sum_{i}^{N} \hat{Q}_{i}\left[i\left(\bar{\phi}_{i} \tilde{D}_{0} \phi_{i}-\phi_{i} \tilde{D}_{0} \bar{\phi}_{i}\right)+\bar{\psi}_{-i} \psi_{-i}+\bar{\psi}_{+i} \psi_{+i}\right]}{2 \sum_{j}^{N} \hat{Q}_{j}^{2}\left|\phi_{j}^{2}\right|}, \\
& \hat{A}_{1}=\frac{\sum_{i}^{N} \hat{Q}_{i}\left[i\left(\bar{\phi}_{i} \tilde{D}_{1} \phi_{i}-\phi_{i} \tilde{D}_{1} \bar{\phi}_{i}\right)-\bar{\psi}_{-i} \psi_{-i}+\bar{\psi}_{+i} \psi_{+i}\right]}{2 \sum_{j} \hat{Q}_{j}^{2}\left|\phi_{j}^{2}\right|},
\end{aligned}
$$

and

$$
\begin{aligned}
& \hat{\sigma}=\frac{\sum_{i=1}^{N}\left[-\hat{Q}_{i}\left(\sum_{c}^{k} \tilde{Q}_{i c} \tilde{\sigma}_{c}\right) \phi_{i} \bar{\phi}_{i}-\hat{Q}_{i} \bar{\psi}_{+i} \psi_{-i}\right]}{\sum_{j=1}^{N} \hat{Q}_{j}^{2} \phi_{j} \bar{\phi}_{j}}, \\
& \overline{\hat{\sigma}}=\frac{\sum_{i=1}^{N}\left[-\hat{Q}_{i}\left(\sum_{c}^{k} \tilde{Q}_{i c} \overline{\tilde{\sigma}}_{c}\right) \phi_{i} \bar{\phi}_{i}-\hat{Q}_{i} \bar{\psi}_{-i} \psi_{+i}\right]}{\sum_{j=1}^{N} \hat{Q}_{j}^{2} \phi_{j} \bar{\phi}_{j}},
\end{aligned}
$$

where

\footnotetext{
${ }^{4}$ These constraints are consistent with the $\mathcal{N}=(2,2)$ supersymmetry of the GLSM action.
}

$$
\begin{aligned}
& \tilde{D}_{\mu} \phi_{i}=\left(\partial_{\mu}+i \sum_{c}^{k} \tilde{Q}_{i c} \tilde{A}_{\mu c}\right) \phi_{i}, \\
& \tilde{D}_{\mu} \bar{\phi}_{i}=\left(\partial_{\mu}-i \sum_{c}^{k} \tilde{Q}_{i c} \tilde{A}_{\mu c}\right) \bar{\phi}_{i} .
\end{aligned}
$$

A good example is that of $X=\mathbb{C} P^{N-1}$, which corresponds to the quotient $\mathbb{C}^{N} / / U(1)$ with charges $\hat{Q}_{i}=1$, and the FI parameter $\hat{r}>0$, which is the Kähler cone of $\mathbb{C} P^{N-1}$. Thus, we should begin with the GLSM (3.8) with $N-k=1$, $\hat{Q}_{i}=1$, and $\hat{r}>0$. In this case, the constraint (3.13) is

$$
\sum_{i}^{N} \bar{\phi}_{i} \phi_{i}=\hat{r}
$$

and is solved by

$$
\begin{aligned}
\phi_{i} & =\frac{Z^{i} \sqrt{\hat{r}} e^{i t}}{\sqrt{1+\sum_{k}^{N-1}\left|Z^{k}\right|^{2}}}, \quad i=1, \ldots, N-1, \\
\phi_{N} & =\frac{\sqrt{\hat{r}} e^{i t}}{\sqrt{1+\sum_{k}^{N-1}\left|Z^{k}\right|^{2}}},
\end{aligned}
$$

where

$$
Z^{i}=\frac{\phi_{i}}{\phi_{N}}
$$

correspond to the inhomogeneous coordinates that parametrize a local patch of $\mathbb{C} P^{N-1}$. Furthermore, the fermionic constraint (3.12) can be solved by

$$
\begin{aligned}
\psi_{i \pm} & =\frac{\psi_{ \pm}^{Z_{i}} \sqrt{\hat{r}} e^{i t}}{\left(1+\sum_{k}^{N-1}\left|Z^{k}\right|^{2}\right)^{\frac{1}{2}}}-\frac{Z^{i}\left(\sum_{l}^{N-1} \psi_{ \pm}^{Z^{l}} \bar{Z}^{l}\right) \sqrt{\hat{r}} e^{i t}}{\left(1+\sum_{k}^{N-1}\left|Z^{k}\right|^{2}\right)^{\frac{3}{2}}} \\
i & =1, \ldots, N-1 \\
\psi_{N \pm} & =\frac{-\sum_{j}^{N-1}\left(\psi_{ \pm}^{Z^{j}} \bar{Z}^{j}\right) \sqrt{\hat{r}} e^{i t}}{\left(1+\sum_{k}^{N-1}\left|Z^{k}\right|^{2}\right)^{\frac{3}{2}}}
\end{aligned}
$$

where $\psi_{ \pm}^{Z^{i}}$ correspond to Grassmann-valued vector fields defined on the aforementioned patch of $\mathbb{C} P^{N-1}$.

Using (3.15) and (3.19), we can show that the terms containing only bosonic fields in the scalar kinetic term of the GLSM $\left(-\sum_{i}^{N} D_{\mu} \phi_{i} D^{\mu} \phi_{i}\right)$ are 


$-\frac{\hat{r}\left(\sum_{j}^{N-1} \partial_{\mu}^{A} Z^{j} \partial^{A \mu} \bar{Z}^{j}\right)}{\left(1+\sum_{i}^{N-1}\left|Z^{i}\right|^{2}\right)}+\frac{\hat{r}\left(\sum_{j}^{N-1} Z^{j} \partial_{\mu}^{A} \bar{Z}^{j}\right)\left(\sum_{k}^{N-1} \bar{Z}^{k} \partial^{A \mu} Z^{k}\right)}{\left(1+\sum_{i}^{N-1}\left|Z^{i}\right|^{2}\right)^{2}}$,

where

$$
\partial_{\mu}^{A} Z^{j}=\partial_{\mu} Z^{j}+i \sum_{c}^{N-1}\left(\tilde{Q}_{j c}-\tilde{Q}_{N c}\right) \tilde{A}_{\mu c} Z^{j} \quad(\text { no sum over } j)
$$

In (3.22), we find the scalar kinetic term given in (2.3) for $X=\mathbb{C} P^{N-1}$ and $G=U(1)^{N-1}$, with the metric on $\mathbb{C} P^{N-1}$ being the standard Fubini-Study metric. ${ }^{5}$ Comparing the covariant derivative (3.23) with the general form given in (2.7), we find that the holomorphic Killing vector fields corresponding to the $U(1)^{N-1}$ isometry on $\mathbb{C} P^{N-1}$ are given by ${ }^{6}$

$$
\tilde{e}_{c}^{j}=i\left(\tilde{Q}_{j c}-\tilde{Q}_{N c}\right) Z^{j}
$$

The term proportional to $\tilde{D}_{c}$ in the GLSM is found to contain the moment map for the $U(1)^{N-1}$ isometry of $\mathbb{C} P^{N-1}$,

$$
\tilde{\mu}_{c}=\frac{-\hat{r}\left(\sum_{i}^{N-1} \tilde{Q}_{i c}\left|Z^{i}\right|^{2}+\tilde{Q}_{N c}\right)}{\left(1+\sum_{k}^{N-1}|Z|^{2}\right)},
$$

via (3.19), and thereby we retrieve the moment map term of (2.4). Similarly, using (3.15) and (3.19), we find that the $\hat{\theta}$ term gives rise to the $B$-field and $C$-field terms of the GNLSM, with

$$
B=-\frac{\hat{\theta}}{\hat{r}} \omega
$$

(where $\omega$ is the Fubini-Study Kähler form) and

$$
C_{c}=-\frac{\hat{\theta}}{\hat{r}} \tilde{\mu}_{c}
$$

as well as the boundary term

$$
-\frac{1}{2 \pi} \int_{\partial \Sigma} \frac{\hat{\theta}}{2 \hat{r}} \sum_{i}^{N}\left(\bar{\psi}_{-i} \psi_{-i}+\bar{\psi}_{+i} \psi_{+i}\right)
$$

where we have maintained its GLSM form for convenience. We shall comment more on this term below.

\footnotetext{
${ }^{5}$ Here, the FI parameter plays the role of the modulus that parametrizes the size of $\mathbb{C} P^{N-1}$.

${ }^{6}$ The fact that the $U(1)^{N-1}$ charges of the inhomogeneous coordinates $Z^{j}$ are given by $\left(\tilde{Q}_{j c}-\tilde{Q}_{N c}\right)$ can also be deduced from (3.20).
}

In a similar manner, we may continue the procedure explained below (3.13) with the help of (3.15), (3.16), (3.19), and (3.21) in order to obtain the complete GNLSM action given in (2.2) for $X=\mathbb{C} P^{N-1}$ and $G=U(1)^{N-1}$, together with the (spurious) boundary term (3.28). This boundary term also occurs in the well-known reduction of GLSMs to NLSMs [19] and can be removed in several ways, including the addition of a boundary term to the GLSM we start with [19], or by a judicious choice of boundary conditions on the fermionic fields. In the following sections, we shall explain how this term is removed when investigating the cases of $A$-type and $B$-type supersymmetry at the boundaries.

Before ending this section, we would like to point out that the classical procedure of obtaining GNLSMs from GLSMs which we have explained above is valid at the quantum level, since the $\hat{e}_{b} \rightarrow \infty$ limit can be taken for the path integral of the GLSM, and functional integration over the auxiliary components of $\hat{V}_{b}$ is equivalent to imposing their algebraic equations of motion as constraints. However, taking renormalization of the FI parameters into account, it can be shown that we may only obtain quantum GNLSMs for Kähler targets with $c_{1}(X) \geq 0$. This is because the renormalization group flow at the one-loop level of the bare FI parameters $\hat{r}_{0 b}$ is

$$
\hat{r}_{0 b}=\hat{r}_{b}(\mu)+\sum_{i=1}^{N} \hat{Q}_{i b} \log \left(\frac{\Lambda_{\mathrm{UV}}}{\mu}\right)
$$

(where $\Lambda_{\mathrm{UV}}$ is an ultraviolet cutoff and $\mu$ is a finite energy scale). As shown in [20], for a basis $e_{b}$ of $H_{2}(X, \mathbb{Z})$, we have $\sum_{i}^{N} \hat{Q}_{i b}=c_{1}(X) \cdot e_{b}$. Then, for a holomorphic curve $m=\sum_{b}^{N-k} m_{b} e_{b}$ in $X$ (i.e., an element of the Mori cone of $X$ )

$\sum_{b}^{N-k} m_{b} \hat{r}_{0 b}=\sum_{b}^{N-k} m_{b} \hat{r}_{b}(\mu)+\sum_{b}^{N-k} m_{b}\left(c_{1}(X) \cdot e_{b}\right) \log \left(\frac{\Lambda_{\mathrm{UV}}}{\mu}\right)$.

For the bare FI parameters to be in the Kähler cone of $X$, the left-hand side of (3.30) ought to be greater than zero. In the continuum limit $\left(\Lambda_{\mathrm{UV}} \rightarrow \infty\right)$, this is impossible if $c_{1}(X) \geq 0$ is not satisfied.

\section{EQUIVARIANT B-BRANES AND THEIR MIRRORS}

We shall apply the techniques discussed above to find boundary actions and boundary conditions in Abelian 
GNLSMs with toric target spaces, $X$, as well as their mirror descriptions. These boundary actions and boundary conditions will correspond to branes in $X$, which we refer to as equivariant branes. We first study the case where $B$ type supersymmetry is preserved at the boundaries of the $I \times \mathbb{R}$ world sheet, since this leads us to make contact with a result found previously by Kapustin et al. [11,21], while in the next section, we shall use similar techniques to study equivariant A-branes. After gaining insights from the study of Abelian equivariant B-branes, we shall then proceed to analyze equivariant B-branes for non-Abelian GNLSMs.

The combination of supercharges that define $B$-type supersymmetry are

$$
\begin{aligned}
& Q_{B}=\bar{Q}_{+}+e^{i \beta} \bar{Q}_{-}, \\
& Q_{B}^{\dagger}=Q_{+}+e^{-i \beta} Q_{-},
\end{aligned}
$$

where $\beta \in \mathbb{R}$. In the following, we shall set $\beta=0$ for simplicity, though it is straightforward to study the $\beta \neq 0$ generalization by the same techniques. From (2.15), we find that the corresponding relations among the supersymmetry transformation parameters are

$$
\begin{aligned}
& \epsilon=\epsilon_{+}=-\epsilon_{-}, \\
& \bar{\epsilon}=\bar{\epsilon}_{+}=-\bar{\epsilon}_{-} .
\end{aligned}
$$

We shall also make use of superfields when discussing boundary conditions, and to this end, the concept of "boundaries" in superspace [10] is useful. For the case at hand, the relevant boundary in superspace is known as " $B$ boundary" and corresponds to

$$
\begin{aligned}
& \theta=\theta^{+}=\theta^{-}, \\
& \bar{\theta}=\bar{\theta}^{+}=\bar{\theta}^{-} .
\end{aligned}
$$

Let us briefly review what is known of ordinary B-branes. For $\mathcal{N}=(2,2)$ NLSMs, the boundary condition needed to preserve $B$-type supersymmetry at the boundaries maps each boundary to a holomorphically embedded complex submanifold of the target space [9]. Such submanifolds can support a holomorphic line bundle, described by the following boundary action:

$S_{\partial \Sigma}=\int_{\partial \Sigma} d x^{0}\left\{A_{M}^{X} \partial_{0} X^{M}-\frac{i}{4} F_{M N}^{X}\left(\psi_{+}^{M}+\psi_{-}^{M}\right)\left(\psi_{+}^{N}+\psi_{-}^{N}\right)\right\}$,

which is $B$-type supersymmetric if $F_{m n}^{X}=F_{\bar{m} \bar{n}}^{X}=0$ [here, we use $(M, N, \ldots)$ as coordinate indices on the holomorphically embedded branes], where $A_{M}^{X}$ corresponds to a connection of a line $[U(1)]$ bundle on each B-brane, and $F_{M N}^{X}$ is the corresponding curvature. Alternatively, when mixed Dirichlet-Neumann boundary conditions are imposed instead of Neumann ones, the boundary action is

$$
S_{\partial \Sigma}=\int_{\partial \Sigma} d x^{0} A_{M}^{X} \partial_{0} X^{M} .
$$

We are interested in the generalizations of (4.4) and (4.5) (and their corresponding boundary conditions) for GNLSMs. These shall be obtained from GLSM boundary conditions and boundary actions, using the methods of Sec. III. In the following, we shall attempt to generalize the boundary action (4.4) to the case of $U(1)^{k}$-GNLSMs with Kähler toric target space, before proceeding to do the same for the boundary action (4.5).

\section{A. Equivariant B-branes on $\mathbb{C}^{N} / / U(1)$ from GLSM}

We shall first proceed to obtain the $B$-type supersymmetric boundary action and boundary conditions for Abelian GNLSMs with toric target spaces of the form $\mathbb{C}^{N} / / U(1)$. We shall focus on obtaining GNLSM boundary conditions corresponding to space-filling branes. To this end, we must impose $B$-type supersymmetric boundary conditions at the GLSM level that include Neumann boundary conditions on the chiral superfields. Hence, we impose the following boundary conditions, which are invariant under $U(1)^{N}$ gauge symmetry:

$$
\begin{aligned}
\mathcal{D}_{+} \Phi_{i} & =\mathcal{D}_{-} \Phi_{i}, \\
\Sigma_{a} & =\bar{\Sigma}_{a}
\end{aligned}
$$

at $B$ boundary, where $\mathcal{D}_{ \pm}=e^{-\sum_{a}^{N} \mathcal{Q}_{i a} V_{a}} D_{ \pm} e^{\sum_{a}^{N} \mathcal{Q}_{i a} V_{a}}$, as well as

$$
F_{01 a}=-e_{a}^{2} \theta_{a}
$$

In components, these boundary conditions are

$$
\begin{aligned}
\psi_{+i}-\psi_{-i} & =0, \\
F_{i} & =0, \\
D_{1} \phi_{i} & =0, \\
D_{1}\left(\psi_{+i}+\psi_{-i}\right) & =0,
\end{aligned}
$$

and

$$
\begin{aligned}
\operatorname{Im}\left(\sigma_{a}\right) & =0, \\
\lambda_{+a}+\lambda_{-a} & =0, \\
\partial_{1} \operatorname{Re}\left(\sigma_{a}\right) & =e_{a}^{2} \theta_{a}, \\
F_{01 a} & =-e_{a}^{2} \theta_{a}, \\
\partial_{1}\left(\lambda_{+a}-\lambda_{-a}\right) & =0, \\
\partial_{1}\left(D_{a}+\partial_{1} \operatorname{Im}\left(\sigma_{a}\right)\right) & =0 .
\end{aligned}
$$


It is crucial to note that these boundary conditions are compatible with the constraints (3.15) and (3.16) which are imposed when taking the $\hat{e} \rightarrow \infty$ limit. We also need to add boundary terms to cancel the $B$-type supersymmetry variations of the bulk theta terms, which gives the $B$-type supersymmetry invariant expression

$$
\begin{aligned}
& \frac{\hat{\theta}}{2 \pi} \int_{\Sigma} \hat{F}_{01} d^{2} x+\frac{\hat{\theta}}{2 \pi} \int_{\partial \Sigma} \frac{(\hat{\sigma}+\overline{\hat{\sigma}})}{2} d x^{0} \\
& \quad+\sum_{c}^{N-1}\left(\frac{\tilde{\theta}_{c}}{2 \pi} \int_{\Sigma} \tilde{F}_{01 c} d^{2} x+\frac{\tilde{\theta}_{c}}{2 \pi} \int_{\partial \Sigma} \frac{\left(\tilde{\sigma}_{c}+\overline{\tilde{\sigma}}_{c}\right)}{2} d x^{0}\right)
\end{aligned}
$$

With the above boundary conditions and boundary terms, the GLSM action is $B$-type supersymmetric at the boundaries.

In addition, we include a boundary action for the $U(1)^{N}$-GLSM given in (3.8), with $N-k=1$. This is given by

$$
\begin{aligned}
S_{\partial \Sigma}= & \frac{\hat{\theta}}{4 \pi \hat{r}} \int_{\partial \Sigma} d x^{0} \sum_{i}^{N}\left(i D_{0} \bar{\phi}_{i} \phi_{i}-i \bar{\phi}_{i} D_{0} \phi_{i}\right. \\
& \left.+\left(\psi_{+i}+\psi_{-i}\right)\left(\bar{\psi}_{+i}+\bar{\psi}_{-i}\right)-\sum_{a}^{N} \mathcal{Q}_{i a}\left(\sigma_{a}+\bar{\sigma}_{a}\right)\left|\phi_{i}\right|^{2}\right)
\end{aligned}
$$

[where the covariant derivatives of the scalar fields are given by (3.9)] and is $B$-type supersymmetric on its own. Its inclusion is necessary to obtain the generalization of the boundary action (4.4), which plays the role of elucidating the geometry of the branes.

Now, recall from [9] that the bulk theta term can be converted into a boundary term in some circumstances. In particular, we have

$$
\frac{\hat{\theta}}{2 \pi} \int_{\Sigma} d^{2} x \hat{F}_{01}=-\frac{\hat{\theta}}{2 \pi} \int_{\partial \Sigma} d x^{0} \hat{A}_{0}
$$

via Stoke's theorem, but this violates gauge invariance. The violation is

$$
\frac{\hat{\theta}}{2 \pi} \int_{\partial \Sigma} d x^{0} \partial_{0} \alpha=\frac{\hat{\theta}}{2 \pi} \int d \alpha=\frac{\hat{\theta}}{2 \pi} 2 \pi m,
$$

where $m \in \mathbb{Z}$. However, if $\hat{\theta} \in 2 \pi \mathbb{Z}$, then (4.13) implies that $\exp \left(-i \frac{\hat{\theta}}{2 \pi} \int_{\partial \Sigma} \hat{A}\right)$ is gauge invariant, and hence, the path integral remains gauge invariant. We shall assume that $\hat{\theta} \in 2 \pi \mathbb{Z}$ hereafter, by setting $\hat{\theta}=2 \pi n$, where $n \in \mathbb{Z}$.
This allows us to write (4.10) and (4.11) as

$$
\begin{aligned}
S_{\partial \Sigma}{ }^{\prime}= & \frac{n}{2 \hat{r}} \int_{\partial \Sigma} d x^{0}\left(\sum_{i}^{N}\left(i \partial_{0} \bar{\phi}_{i} \phi_{i}-i \bar{\phi}_{i} \partial_{0} \phi_{i}\right)\right. \\
& +\sum_{i}^{N}\left(\psi_{+i}+\psi_{-i}\right)\left(\bar{\psi}_{+i}+\bar{\psi}_{-i}\right) \\
& +2 \hat{A}_{0}\left(\sum_{i}^{N} \hat{Q}_{i}\left|\phi_{i}\right|^{2}-\hat{r}\right)-(\hat{\sigma}+\overline{\hat{\sigma}})\left(\sum_{i}^{N} \hat{Q}_{i}\left|\phi_{i}\right|^{2}-\hat{r}\right) \\
& \left.+2 \sum_{c}^{N-1} \tilde{A}_{0 c} \sum_{i}^{N} \tilde{Q}_{i c}\left|\phi_{i}\right|^{2}-\sum_{c}^{N-1}\left(\tilde{\sigma}_{c}+\overline{\tilde{\sigma}}_{c}\right) \sum_{i}^{N} \tilde{Q}_{i c}\left|\phi_{i}\right|^{2}\right) \\
& +\sum_{c}^{N-1}\left(\frac{\tilde{\theta}_{c}}{2 \pi} \int_{\Sigma} \tilde{F}_{01 c} d^{2} x+\frac{\tilde{\theta}_{c}}{2 \pi} \int_{\partial \Sigma} \frac{\left(\tilde{\sigma}_{c}+\overline{\tilde{\sigma}}_{c}\right)}{2} d x^{0}\right) .
\end{aligned}
$$

Taking the $\hat{e} \rightarrow \infty$ limit results in the corresponding vector multiplet components becoming auxiliary (see Sec. III). Integrating $\hat{D}$ out of the bulk action imposes the condition

$$
\sum_{i}^{N} \hat{Q}_{i}\left|\phi_{i}\right|^{2}-\hat{r}=0
$$

and this results in the second line of (4.14) vanishing. Integrating out the rest of the components in the vector multiplet gives several more constraints, the one relevant to the boundary action being ${ }^{7}$

$$
\sum_{i}^{N} \hat{Q}_{i} \bar{\phi}_{i} \psi_{ \pm i}=0
$$

As explained in [9], the first term in (4.14) is nothing but the Hermitian connection

$$
A_{I}^{X} d X^{I}=-n \frac{i}{2} \frac{\sum_{i=1}^{N} \bar{\phi}_{i} \stackrel{\leftrightarrow}{d} \phi_{i}}{\sum_{i=1}^{N} \hat{Q}_{i}\left|\phi_{i}\right|^{2}}
$$

of $\mathcal{O}_{X}(-n)$ on the toric manifold $X=\mathbb{C}^{N} / / U(1)$, since it transforms under $U(1)$ gauge transformations $\left(\phi_{i} \rightarrow e^{i \hat{Q}_{i} \alpha} \phi_{i}\right)$ as

$$
A_{I}^{X} d X^{I} \rightarrow A_{I}^{X} d X^{I}-(-n) d \alpha .
$$

Here, $\mathcal{O}_{X}(-n)$ is the holomorphic line bundle on $X$ with $\int_{X} c_{1}\left(\mathcal{O}_{X}(-n)\right)=-n$.

Next, to find the explicit $B$-type GNLSM boundary conditions and boundary action, we must find parametrizations that satisfy (4.15) and (4.16). Let us study our usual example of $\mathbb{C} P^{N-1}$. Using the parametrizations (3.19) and

\footnotetext{
${ }^{7}$ It is important to integrate out $\hat{D}$ before integrating out $\hat{A}_{0}$; otherwise, the algebraic equation of motion of $\hat{A}_{0}$ given in (3.15) will be modified by a boundary term (see footnote 3 ).
} 
(3.21), as well as the constraints (3.15) and (3.16), the boundary conditions become

$$
\begin{aligned}
\psi_{+}^{Z^{i}}-\psi_{-}^{Z^{i}} & =0, \\
F^{Z^{i}} & =0, \\
\partial_{1}^{A} Z^{i} & =0 \\
\phi^{*} \nabla_{1}^{A}\left(\psi_{+}^{Z^{i}}+\psi_{-}^{Z^{i}}\right) & =0,
\end{aligned}
$$

and

$$
\begin{aligned}
\operatorname{Im}\left(\tilde{\sigma}_{c}\right) & =0, \\
\tilde{\lambda}_{+c}+\tilde{\lambda}_{-c} & =0, \\
\partial_{1} \operatorname{Re}\left(\tilde{\sigma}_{c}\right) & =\tilde{e}_{c}^{2} \tilde{\theta}_{c}, \\
\tilde{F}_{01 c} & =-\tilde{e}_{c}^{2} \tilde{\theta}_{c}, \\
\partial_{1}\left(\tilde{\lambda}_{+c}-\tilde{\lambda}_{-c}\right) & =0 \\
\partial_{1}\left(\tilde{D}_{c}+\partial_{1} \operatorname{Im}\left(\tilde{\sigma}_{c}\right)\right) & =0
\end{aligned}
$$

which are invariant under the $U(1)^{N-1}$ gauge symmetry and satisfy the $B$-type supersymmetry transformations obtained from (2.13) and (2.14). Moreover, these boundary conditions result in the vanishing of the expressions (2.16) and (2.17), thus ensuring the preservation of $B$ type supersymmetry at the boundaries. Note that the expression (2.18) does not occur when performing a supersymmetry variation, since the $B$-field and $C$-field terms do not appear in the action of the GNLSM, as we have used the bulk $\hat{\theta}$ term of the corresponding GLSM in the construction of our boundary action via (4.12). The spurious boundary term (3.28) also does not occur, for the same reason. In addition, the Neumann boundary conditions on $Z^{i}$ imply that the equivariant B-brane wraps the entire target space, $\mathbb{C} P^{N-1}$; i.e., it is space filling.

Next, let us find the explicit form of the boundary action. The parametrizations (3.19) and (3.21) give

$$
\begin{aligned}
S_{\partial \Sigma}^{\prime}= & \int_{\partial \Sigma} d x^{0}\left\{A_{j}^{X} \partial_{0} X^{j}+A_{\bar{\jmath}}^{X} \partial_{0} \bar{X}^{\bar{j}}-\sum_{c}^{N-1} i R_{c} \tilde{\mathcal{A}}_{c}+n \partial_{0} t-\frac{i}{2} F_{j \bar{k}}^{X}\left(\psi_{+}^{j}+\psi_{-}^{j}\right)\left(\bar{\psi}_{+}^{\bar{k}}+\bar{\psi}_{-}^{\bar{k}}\right)\right\} \\
& +\sum_{c}^{N-1}\left(\frac{\tilde{\theta}_{c}}{2 \pi} \int_{\Sigma} \tilde{F}_{01 c} d^{2} x+\frac{\tilde{\theta}_{c}}{2 \pi} \int_{\partial \Sigma} \frac{\left(\tilde{\sigma}_{c}+\overline{\tilde{\sigma}}_{c}\right)}{2} d x^{0}\right),
\end{aligned}
$$

where $X^{j}=Z^{j}, \psi_{ \pm}^{j}=\psi_{ \pm}^{Z^{j}}$,

$$
\begin{aligned}
A_{j}^{X} & =-n \frac{i}{2} \frac{\bar{Z}^{j}}{\left(1+\sum_{k}^{N-1}\left|Z^{k}\right|^{2}\right)}, \\
A_{\bar{J}}^{X} & =n \frac{i}{2} \frac{Z^{j}}{\left(1+\sum_{k}^{N-1}\left|Z^{k}\right|^{2}\right)}
\end{aligned}
$$

are the components of the connection of $\mathcal{O}_{\mathbb{C} P^{N-1}}(-n)$, while its curvature is

$$
F_{j \bar{k}}=n \omega_{j \bar{k}},
$$

where $\omega_{j \bar{k}}=i g_{j \bar{k}}$ are the components of the normalized ${ }^{8}$ Fubini-Study Kähler form of $\mathbb{C} P^{N-1}$,

$$
\begin{aligned}
\omega= & i \frac{\left(\sum_{j}^{N-1} d Z^{j} \wedge d \bar{Z}^{j}\right)}{\left(1+\sum_{k}^{N-1}\left|Z^{k}\right|^{2}\right)} \\
& -i \frac{\left(\sum_{l}^{N-1} \bar{Z}^{l} d Z^{l}\right) \wedge\left(\sum_{j}^{N-1} Z^{j} d \bar{Z}^{j}\right)}{\left(1+\sum_{k}^{N-1}\left|Z^{k}\right|^{2}\right)^{2}} .
\end{aligned}
$$

Besides the supersymmetrized $\tilde{\theta}$ terms, the only other term with vector multiplet fields is

\footnotetext{
${ }^{8}$ Unlike (4.24), the Fubini-Study metric that appears in the bulk GNLSM action contains the FI parameter, $\hat{r}$, which is the size modulus of the Fubini-Study metric; see (3.22).
}

$$
-\sum_{c}^{N-1} i R_{c} \tilde{\mathcal{A}}_{c}
$$

where

$$
\tilde{\mathcal{A}}_{c}=-i\left(\tilde{A}_{0 c}-\frac{\left(\tilde{\sigma}_{c}+\overline{\tilde{\sigma}}_{c}\right)}{2}\right)
$$

and

$$
R_{c}=\frac{-n\left(\sum_{i}^{N-1} \tilde{Q}_{i c}\left|Z^{i}\right|^{2}+\tilde{Q}_{N c}\right)}{\left(1+\sum_{k}^{N-1}|Z|^{2}\right)} .
$$

The expression (4.21) is gauge invariant under the unbroken $U(1)^{N-1}$ symmetry. ${ }^{9}$ The $n \partial_{0} t$ term reflects the fact that a $U(1)$ gauge symmetry is broken at the boundaries, and it can be removed via the gauge transformation (4.18), with $\alpha=-t$. Now, we must remove this term, since $t$ is not a coordinate of the $\mathbb{C} P^{N-1}$ target space, but rather locally parametrizes the Hopf fiber over $\mathbb{C} P^{N-1}$, which gives rise to the sphere $S^{N+1}$ defined by Eq. (3.18). Furthermore, it is not a field that appears in the bulk theory, and has no supersymmetry transformation, leaving us unable to test the

\footnotetext{
${ }^{9}$ Note that, from the local parametrizations (3.19) and (3.21), we can see that the $U(1)^{N-1}$ gauge transformation of $t$ is $\delta t=\tilde{Q}_{N c} \alpha$, since we know that the $U(1)^{N-1}$ charge of $Z^{i}$ is $\tilde{Q}_{1 c}-\tilde{Q}_{N c}$.
} 
supersymmetry of the boundary action. Thus, we shall gauge away $n \partial_{0} t$. However, doing so will break the $U(1)^{N-1}$ symmetry of our GNLSM at the boundaries. ${ }^{10}$

The cure to this broken symmetry is via the supersymmetrized $\tilde{\theta}$ terms, as follows. Setting $\tilde{\theta}_{c}=2 \pi n \tilde{Q}_{N c}$, we have

$$
\begin{aligned}
& \sum_{c}^{N-1}\left(\frac{\tilde{\theta}_{c}}{2 \pi} \int_{\Sigma} \tilde{F}_{01 c} d^{2} x+\frac{\tilde{\theta}_{c}}{2 \pi} \int_{\partial \Sigma} \frac{\left(\tilde{\sigma}_{c}+\overline{\tilde{\sigma}}_{c}\right)}{2} d x^{0}\right) \\
& =\sum_{c}^{N-1}\left(-n \tilde{Q}_{N c} \int_{\partial \Sigma} \tilde{A}_{0 c} d x^{0}+n \tilde{Q}_{N c} \int_{\partial \Sigma} \frac{\left(\tilde{\sigma}_{c}+\overline{\tilde{\sigma}_{c}}\right)}{2} d x^{0}\right) \\
& =\sum_{c}^{N-1}\left(-i n \tilde{Q}_{N c} \int_{\partial \Sigma} \tilde{\mathcal{A}}_{c} d x^{0}\right)
\end{aligned}
$$

since both $n$ and the charge $\tilde{Q}_{N c}$ are integers, as explained below (4.12). Then, the final boundary action takes the form

$$
S_{\partial \Sigma}{ }^{\prime}=\int_{\partial \Sigma} d x^{0}\left\{A_{j}^{X} \partial_{0} X^{j}+A_{\bar{\jmath}}^{X} \partial_{0} \bar{X}^{\bar{j}}-\sum_{c}^{N-1} i \tilde{R}_{c} \tilde{\mathcal{A}}_{c}-\frac{i}{2} F_{j \bar{k}}^{X}\left(\psi_{+}^{j}+\psi_{-}^{j}\right)\left(\bar{\psi}_{+}^{\bar{k}}+\bar{\psi}_{-}^{\bar{k}}\right)\right\},
$$

where $^{11}$

$$
\begin{aligned}
\tilde{R}_{c} & =\frac{-n\left(\sum_{i}^{N-1}\left(\tilde{Q}_{i c}-\tilde{Q}_{N c}\right)\left|Z^{i}\right|^{2}\right)}{\left(1+\sum_{k}^{N-1}\left|Z^{k}\right|^{2}\right)} \\
& =-A_{i}^{X} \tilde{e}_{c}^{i}-A_{\bar{J}}^{X} \tilde{\tilde{e}}_{c}^{\bar{J}} \\
& =-l_{\tilde{e}_{c}} A^{X} .
\end{aligned}
$$

Invariance of the boundary action (4.29) under the $B$-type supersymmetry transformations [given by (2.13) and (2.14) for $\left.\epsilon_{+}=-\epsilon_{-}\right]$holds since

$$
d \tilde{R}=l_{\tilde{e}} F^{X} .
$$

This is known as the equivariant Bianchi identity and implies that the line bundle $\mathcal{O}_{\mathbb{C} P^{N-1}}(-n)$ has $U(1)^{N-1}$-equivariant structure, ${ }^{12}$ for which $\tilde{R}_{c}$ is the moment [22,23].

The equivariant Bianchi identity is, in fact, a restatement of the $U(1)^{N-1}$ invariance of the connection,

$$
\mathcal{L}_{\tilde{e}} A^{X}=0 .
$$

Now, rewriting the boundary action (4.29) as

$$
S_{\partial \Sigma}{ }^{\prime}=\int_{\partial \Sigma} d x^{0}\left\{A_{j}^{X} \partial_{0}^{A} X^{j}+A_{\bar{j}}^{X} \partial_{0}^{A} \bar{X}^{\bar{j}}+\sum_{c}^{N-1} \tilde{R}_{c} \frac{\left(\tilde{\sigma}_{c}+\overline{\tilde{\sigma}}_{c}\right)}{2}-\frac{i}{2} F_{j \bar{k}}^{X}\left(\psi_{+}^{j}+\psi_{-}^{j}\right)\left(\bar{\psi}_{+}^{\bar{k}}+\bar{\psi}_{-}^{\bar{k}}\right)\right\}
$$

facilitates the proof that it is invariant under the gauge transformations given in (2.11) and (2.12) for $G=U(1)^{N-1}$. The variation is

$$
\delta S_{\partial \Sigma}{ }^{\prime}=\sum_{a}^{N-1} \alpha_{a} \int_{\partial \Sigma} d x^{0}\left\{\mathcal{L}_{\tilde{e}_{a}} A_{j}^{X} \partial_{0}^{A} X^{j}+\mathcal{L}_{\tilde{e}_{a}} A_{\bar{j}}^{X} \partial_{0}^{A} \bar{X}^{\bar{j}}+\sum_{c}^{N-1} l_{\tilde{e}_{a}} d \tilde{R}_{c} \frac{\left(\tilde{\sigma}_{c}+\overline{\tilde{\sigma}}_{c}\right)}{2}-\frac{i}{2} \mathcal{L}_{\tilde{e}_{a}} F_{j \bar{k}}^{X}\left(\psi_{+}^{j}+\psi_{-}^{j}\right)\left(\bar{\psi}_{+}^{\bar{k}}+\bar{\psi}_{-}^{\bar{k}}\right)\right\},
$$

\footnotetext{
${ }^{10}$ This $U(1)^{N-1}$ gauge symmetry is not broken if we only require that it holds for the path integral and not necessarily the action. Even in that case, the following steps help make the geometric properties of the brane obvious.

${ }^{11}$ The boundary conditions (4.20) and the boundary action (4.29) result in equations of motion that are modified by boundary terms, for some of the fields.

${ }^{12}$ The $G$-equivariant Bianchi identity is equivalent to the $G$ invariance of the connection, $A$, of the bundle [Eq. (4.32)], which implies that the covariant derivative $d+A$ is $G$ invariant, and this defines a $G$-equivariant bundle; see [22], Sec. 3.2.
} 
which vanishes using (4.32), as well as the identities $\mathcal{L}_{\tilde{e}} F^{X}=0$ and $\mathcal{L}_{\tilde{e}} \tilde{R}=l_{\tilde{e}} d \tilde{R}=0 .{ }^{13}$

We have thus found $B$-type supersymmetric and $U(1)^{N-1}$ gauge invariant boundary conditions and boundary interactions corresponding to an equivariant B-brane in $\mathbb{C} P^{N-1}$, which is a space-filling brane supporting the holomorphic line bundle $\mathcal{O}_{\mathbb{C} P^{N-1}}(-n)$ with $U(1)^{N-1}$ equivariant structure. We may follow a procedure analogous to that presented above for $\mathbb{C} P^{N-1}$ in order to describe an equivariant B-brane in a toric manifold $X=\mathbb{C}^{N} / / U(1)$ (by choosing different values for $\hat{Q}_{i}$ ), which would be a space-filling brane supporting the holomorphic line bundle $\mathcal{O}_{X}(-n)$ with $U(1)^{N-1}$-equivariant structure.

The GNLSM boundary action (4.29) that we have derived from the GLSM expressions (4.10) and (4.11) is a special case of the more general boundary Wilson line found by Kapustin et al. [11,21], using a B-twisted topological non-Abelian GNLSM, with gauge group $G$ and target space $X$, i.e., a gauged $B$ model. This boundary Wilson line corresponds to a graded $G$-equivariant holomorphic vector bundle. As explained in [11,21], in some cases, the category of branes defined by the boundary Wilson line is equivalent to $D_{G_{C}}^{b}(\operatorname{Coh}(X))$, the bounded, derived category of $G_{\mathbb{C}}$-equivariant coherent sheaves on the target space, $X$. This occurs if $X$ has a $G$-resolution property; i.e., any $G$-equivariant coherent sheaf on $X$ has a $G$-equivariant resolution by $G$-equivariant holomorphic vector bundles. This property, however, does not hold for general complex manifolds. Nevertheless, even for such spaces where it does not hold, it is believed that the full category of equivariant B-branes is still $D_{G_{\mathrm{C}}}^{b}(\mathrm{Coh}(X))$, where the GNLSMs for these spaces require more general boundary actions corresponding to differential graded (DG) modules over the Dolbeault DG algebra of $X$, instead of holomorphic bundles.

In our construction, we have found Abelian equivariant B-branes that wrap toric manifolds given by the quotient $X=\mathbb{C}^{N} / / U(1)$, and that support the $U(1)^{N-1}$-equivariant holomorphic line bundle $\mathcal{O}_{X}(-n)$. In the language of algebraic geometry, $\mathcal{O}_{X}(-n)$ is a locally free sheaf of rank 1 and is, in fact, one of the simplest objects of $D^{b}(\operatorname{Coh}(X))$ ([24], page 56). The additional $U(1)^{N-1}$-equivariant structure then implies that the equivariant B-branes we have found are objects in $D_{\left(\mathbb{C}^{\times}\right)^{N-1}}^{b}(\operatorname{Coh}(X))$, the bounded, derived category of $\left(\mathbb{C}^{\times}\right)^{N-1}$-equivariant coherent sheaves on $X{ }^{14}$ Of course, we have not constructed all the objects in the category.

In particular, we have not constructed non-space-filling equivariant B-branes. The latter, i.e., equivariant B-branes of lower dimension, should exist, in analogy with the NLSM case, although we shall not attempt to derive them from GLSMs here. The path to doing so is via Hori's construction of non-space-filling ordinary B-branes from GLSMs [10]. Using the same GLSM used there, but with gauge group generalized to $U(1)^{N}$, we should be able to derive the relevant GNLSM boundary action and boundary conditions, as we have done for space-filling equivariant B-branes in this section.

\section{B. Equivariant B-branes on $\mathbb{C}^{N} / / U(1)^{N-k}$ from GLSM}

The prior discussion can be generalized to the case of general Kähler toric manifolds, i.e., $X=\mathbb{C}^{N} / / U(1)^{N-k}$. We impose the $B$-type supersymmetric boundary conditions (4.6) and (4.7) on the GLSM (for $N-k>1$ ), which include the purely Neumann boundary conditions on $\phi_{i}$, while also supersymmetrizing the GLSM theta terms

$$
\sum_{b}^{N-k}\left(\frac{\hat{\theta}_{b}}{2 \pi} \int_{\Sigma} \hat{F}_{01 b} d^{2} x+\frac{\hat{\theta}_{b}}{2 \pi} \int_{\partial \Sigma} \frac{\left(\hat{\sigma}_{b}+\overline{\hat{\sigma}}_{b}\right)}{2} d x^{0}\right)+\sum_{c}^{k}\left(\frac{\tilde{\theta}_{c}}{2 \pi} \int_{\Sigma} \tilde{F}_{01 c} d^{2} x+\frac{\tilde{\theta}_{c}}{2 \pi} \int_{\partial \Sigma} \frac{\left(\tilde{\sigma}_{c}+\overline{\tilde{\sigma}}_{c}\right)}{2} d x^{0}\right) .
$$

This preserves $B$-type supersymmetry at the boundaries. In addition, the $B$-type supersymmetric GLSM boundary action needed is

$$
S_{\partial \Sigma}=\frac{\theta^{\prime}}{4 \pi r^{\prime}} \int_{\partial \Sigma} d x^{0} \sum_{i}^{N}\left(i D_{0} \bar{\phi}_{i} \phi_{i}-i \bar{\phi}_{i} D_{0} \phi_{i}+\left(\psi_{+i}+\psi_{-i}\right)\left(\bar{\psi}_{+i}+\bar{\psi}_{-i}\right)-\sum_{a}^{N} \mathcal{Q}_{i a}\left(\sigma_{a}+\bar{\sigma}_{a}\right)\left|\phi_{i}\right|^{2}\right),
$$

where $\theta^{\prime}=2 \pi n^{\prime}\left(n^{\prime} \in \mathbb{Z}\right)$ and $r^{\prime} \in \mathbb{R}$. In addition, we ought to set $\hat{\theta}_{b}=2 \pi \hat{n}_{b}$, where $\hat{n}_{b} \in \mathbb{Z}$, and we need to impose the condition

for all values of $b$

$$
\frac{\theta^{\prime}}{r^{\prime}}=\frac{\hat{\theta}_{b}}{\hat{r}_{b}}
$$

\footnotetext{
${ }^{13}$ If we require only $U(1)^{N-1}$ gauge invariance of the path integral, then we are free to choose $\tilde{\theta}_{c}=2 \pi m_{c}$ for any integer $m_{c}$, and we would still derive a boundary action which is $B$-type supersymmetry invariant, as well as gauge invariant mod $2 \pi \mathbb{Z}$.. This freedom is merely a reflection of the fact that the moment in the equivariant Bianchi identity (4.31) is defined only up to a constant.

${ }^{14}$ The algebraic torus $\left(\mathbb{C}^{\times}\right)^{N-1}$ is the complexification of $U(1)^{N-1}$.
} 
This allows us to write (4.36) and (4.35) as

$$
\begin{aligned}
S_{\partial \Sigma}{ }^{\prime}= & \frac{n^{\prime}}{2 r^{\prime}} \int_{\partial \Sigma} d x^{0}\left(\sum_{i}^{N}\left(i \partial_{0} \bar{\phi}_{i} \phi_{i}-i \bar{\phi}_{i} \partial_{0} \phi_{i}\right)+\sum_{i}^{N}\left(\psi_{+i}+\psi_{-i}\right)\left(\bar{\psi}_{+i}+\bar{\psi}_{-i}\right)\right. \\
& +2 \sum_{b}^{N-k} \hat{A}_{0 b}\left(\sum_{i}^{N} \hat{Q}_{i b}\left|\phi_{i}\right|^{2}-\hat{r}_{b}\right)-\sum_{b}^{N-k}\left(\hat{\sigma}_{b}+\overline{\hat{\sigma}}_{b}\right)\left(\sum_{i}^{N} \hat{Q}_{i b}\left|\phi_{i}\right|^{2}-\hat{r}_{b}\right) \\
& \left.+2 \sum_{c}^{k} \tilde{A}_{0 c} \sum_{i}^{N} \tilde{Q}_{i c}\left|\phi_{i}\right|^{2}-\sum_{c}^{k}\left(\tilde{\sigma}_{c}+\overline{\tilde{\sigma}}_{c}\right) \sum_{i}^{N} \tilde{Q}_{i c}\left|\phi_{i}\right|^{2}\right) \\
& +\sum_{c}^{k}\left(\frac{\tilde{\theta}_{c}}{2 \pi} \int_{\Sigma} \tilde{F}_{01 c} d^{2} x+\frac{\tilde{\theta}_{c}}{2 \pi} \int_{\partial \Sigma} \frac{\left(\tilde{\sigma}_{c}+\overline{\tilde{\sigma}}_{c}\right)}{2} d x^{0}\right) .
\end{aligned}
$$

Taking the $\hat{e}_{b} \rightarrow \infty$ limit allows us to integrate $\hat{D}_{b}$ out of the action, which imposes the constraints (3.13), and the second line in (4.38) vanishes. Integrating out the other components of the vector multiplets, $\hat{V}_{b}$, then imposes (3.12) on the entire action, as well (3.10) and (3.11) on the bulk action. Then, to find the explicit boundary action, one needs to use parametrizations that satisfy (3.12) and (3.13). The explicit boundary conditions are also found using these parametrizations, together with (3.10) and (3.11).

We can identify the first term in (4.38) as the Hermitian connection,

$$
A_{I}^{X} d X^{I}=-\frac{n^{\prime}}{r^{\prime}} \frac{i}{2} \sum_{i=1}^{N} \bar{\phi}_{i} \stackrel{\leftrightarrow}{d} \phi_{i}
$$

of the holomorphic line bundle $\bigotimes_{b=1}^{N-k} \mathcal{O}_{X}\left(-\hat{n}_{b}\right)=$ $\mathcal{O}_{X}\left(\sum_{b=1}^{N-k}\left(-\hat{n}_{b}\right)\right)$ over $\mathbb{C}^{N} / / U(1)^{N-k}$, since it transforms under $U(1)^{N-k}$ gauge transformations $\left(\phi_{i} \rightarrow\right.$ $\left.e^{i \sum_{b=1}^{N-k} \hat{Q}_{i b} \alpha_{b}} \phi_{i}\right)$ as

$$
A_{I}^{X} d X^{I} \rightarrow A_{I}^{X} d X^{I}-\sum_{b=1}^{N-k}\left(-\hat{n}_{b}\right) d \alpha_{b}
$$

and setting $\alpha_{b}=\alpha$, we retrieve the $U(1)$ gauge transformation of the connection of $\otimes_{b=1}^{N-k} \mathcal{O}_{X}\left(-\hat{n}_{b}\right)$. Both supersymmetry invariance and gauge invariance under the residual $U(1)^{k}$ gauge symmetry of the GNLSM would then require that this line bundle has $U(1)^{k}$-equivariant structure. Moreover, we would be able to identify the equivariant B-branes we have found as objects in $D_{\left(\mathbb{C}^{\times}\right)^{k}}^{b}(\operatorname{Coh}(X))$, the bounded, derived category of $\left(\mathbb{C}^{\times}\right)^{k}$-equivariant coherent sheaves on $X$.

The simplest example would be that of the $U(1)^{2}$ equivariant holomorphic line bundle $\otimes_{b=1}^{2} \mathcal{O}_{X}\left(-\hat{n}_{b}\right)$ over $X=\mathbb{C} P^{1} \times \mathbb{C} P^{1}$, which just corresponds to two copies of the boundary action given in (4.29), with $N=2 .{ }^{15}$ One can even consider equivariant B-branes on fibrations of $\mathbb{C} P^{1}$ over $\mathbb{C} P^{1}$ known as Hirzebruch surfaces, using GLSMs with appropriately charged scalar fields. It is worth noting that the derived categories of $\mathbb{C}^{\times}$-equivariant coherent sheaves over $\mathbb{C} P^{1}$, Hirzebruch surfaces, $\mathbb{C} P^{1}$ fibered over Hirzebruch surfaces, etc., provide a construction of Khovanov homology [25].

\section{Alternative formulation}

We shall now derive the alternative formulation of Abelian equivariant B-branes, in terms of a boundary action that generalizes (4.5), as well as the relevant boundary conditions. To derive the boundary action for a GNLSM with $X=\mathbb{C}^{N} / / U(1)$, we start with the $U(1)^{N_{-}}$ GLSM boundary action

$$
S_{\partial \Sigma}=\frac{\hat{\theta}}{4 \pi \hat{r}} \int_{\partial \Sigma} d x^{0} \sum_{i}^{N}\left(i D_{0} \bar{\phi}_{i} \phi_{i}-i \bar{\phi}_{i} D_{0} \phi_{i}\right)
$$

where the covariant derivatives of the scalar fields are given by (3.9). $B$-type supersymmetry invariance of the $U(1)^{N}$-GLSM at the boundaries of the world sheet first requires that we impose

$$
\begin{gathered}
e^{-i \hat{\gamma} \mathcal{D}_{+} \Phi_{i}}=e^{i \hat{\gamma}} \mathcal{D}_{-} \Phi_{i} \\
e^{i \gamma_{a} \Sigma_{a}}=e^{-i \gamma_{a} \bar{\Sigma}_{a}}
\end{gathered}
$$

at $B$ boundary, where $\mathcal{D}_{ \pm}=e^{-\sum_{a}^{N} \mathcal{Q}_{i a} V_{a}} D_{ \pm} e^{\sum_{a}^{N} \mathcal{Q}_{i a} V_{a}}$, while $\hat{\gamma}$ and $\gamma_{a}$ are the phases of $\hat{t}=|\hat{t}| e^{i \hat{\gamma}}$ and $t_{a}=\left|t_{a}\right| e^{i \gamma_{a}}$, respectively. Second, we also ought to impose

\footnotetext{
${ }^{15}$ In fact, for toric manifolds that are Cartesian products like $X=\mathbb{C} P^{1} \times \mathbb{C} P^{1}$, the complete decoupling of the two boundary actions means that we no longer need the constraint (4.37).
} 


$$
\frac{\hat{\theta}}{\hat{r}}=\frac{\theta_{a}}{r_{a}}
$$

and

$$
\hat{\gamma}=\gamma_{a}
$$

Then, in components, (4.42) and (4.43) become

$$
\begin{aligned}
e^{-i \hat{\gamma}} \psi_{+i}-e^{i \hat{\gamma}} \psi_{-i} & =0, \\
F_{i} & =0, \\
\cos (\hat{\gamma}) D_{1} \phi_{i}-i \sin (\hat{\gamma}) D_{0} \phi_{i} & =0, \\
\cos (\hat{\gamma}) D_{1}\left(\psi_{+i}+\psi_{-i}\right)-i \sin (\hat{\gamma}) D_{0}\left(\psi_{+i}+\psi_{-i}\right)-\cos (\hat{\gamma}) \sum_{a}^{N} Q_{i a}\left(\lambda_{+a}+\lambda_{-a}\right) \phi_{i} & =0,
\end{aligned}
$$

and

$$
\begin{aligned}
\operatorname{Im}\left(e^{i \hat{\gamma}} \sigma_{a}\right) & =0, \\
e^{-i \hat{\gamma}} \lambda_{+a}+e^{i \hat{\gamma}} \lambda_{-a} & =0, \\
\partial_{1} \operatorname{Re}\left(e^{i \hat{\gamma}} \sigma_{a}\right)+\cos (\hat{\gamma}) F_{01 a}-\sin (\hat{\gamma}) D_{a} & =0,
\end{aligned}
$$

which includes the mixed Dirichlet-Neumann boundary condition on the scalar fields $\phi_{i}$. Finally, for complete boundary $B$-type supersymmetry invariance, we must impose the boundary condition

$$
\frac{F_{01 a}}{e_{a}^{2}}=-\theta_{a}+\hat{\theta} \frac{\sum_{i}^{N} \mathcal{Q}_{i a}\left|\phi_{i}\right|^{2}}{\hat{r}}
$$

as well as integrate $D_{a}$ out of the action to obtain its algebraic equation of motion

$$
\frac{D_{a}}{e_{a}^{2}}=r_{a}-\sum_{i}^{N} \mathcal{Q}_{i a}\left|\phi_{i}\right|^{2}
$$

which holds on the entire world sheet. ${ }^{16}$ The condition (4.48) further implies two more boundary conditions via $B$ type supersymmetry. All the boundary conditions above ensure the locality of the equations of motion derived from the action.

Now, setting $\hat{\theta}=2 \pi n$, the relevant action that consists of (4.41) together with the theta terms is

$$
\begin{aligned}
S_{\partial \Sigma^{\prime}}= & \frac{n}{2 \hat{r}} \int_{\partial \Sigma} d x^{0}\left(\sum_{i}^{N}\left(i \partial_{0} \bar{\phi}_{i} \phi_{i}-i \bar{\phi}_{i} \partial_{0} \phi_{i}\right)\right. \\
& +2 \hat{A}_{0}\left(\sum_{i}^{N} \hat{Q}_{i}\left|\phi_{i}\right|^{2}-\hat{r}\right) \\
& \left.+2 \sum_{c}^{N-1} \tilde{A}_{0 c} \sum_{i}^{N} \tilde{Q}_{i c}\left|\phi_{i}\right|^{2}\right)+\sum_{c}^{N-1}\left(\frac{\tilde{\theta}_{c}}{2 \pi} \int_{\Sigma} \tilde{F}_{01 c} d^{2} x\right) .
\end{aligned}
$$

The term proportional to $\hat{A}_{0}$ vanishes in the $\hat{e} \rightarrow \infty$ limit using the equation of motion for $\hat{D}$ given in (4.49), while the constraints that arise from subsequently integrating out the rest of the vector multiplet $\hat{V}$ do not affect the boundary action. As before, we obtain the Hermitian connection (4.17) of $\mathcal{O}_{X}(-n)$ on the toric manifold $X=\mathbb{C}^{N} / / U(1)$. For $X=\mathbb{C} P^{N-1}$, we can use the parametrizations (3.19), and (4.50) becomes

$$
\begin{aligned}
S_{\partial \Sigma}{ }^{\prime}= & \int_{\partial \Sigma} d x^{0}\left\{A_{j}^{X} \partial_{0} X^{j}+A_{\bar{J}}^{X} \partial_{0} \bar{X}^{\bar{J}}-\sum_{c}^{N-1} i R_{c} \tilde{A}_{c}+n \partial_{0} t\right\} \\
& +\sum_{c}^{N-1}\left(\frac{\tilde{\theta}_{c}}{2 \pi} \int_{\Sigma} \tilde{F}_{01 c} d^{2} x\right)
\end{aligned}
$$

with $A_{I}$ given in (4.22) and $R_{c}$ given in (4.27). Then, gauging away the $n \partial_{0} t$ term, and setting $\tilde{\theta}_{c}=2 \pi n \tilde{Q}_{N c}$, we arrive at the boundary action

$$
S_{\partial \Sigma}{ }^{\prime}=\int_{\partial \Sigma} d x^{0}\left\{A_{j}^{X} \partial_{0} X^{j}+A_{\bar{\jmath}}^{X} \partial_{0} \bar{X}^{\bar{j}}-\sum_{c}^{N-1} i \tilde{R}_{c} \tilde{\mathcal{A}}_{c}\right\},
$$

\footnotetext{
${ }^{16}$ The constraints (4.45), (4.48), and (4.49) result in the third equation of (4.47) becoming $\partial_{1} \operatorname{Re}\left(e^{i \hat{\gamma}} \sigma_{a}\right)=0$.
}

where $\tilde{R}_{c}$ is the moment given by (4.30). The boundary action can be rewritten concisely as 


$$
S_{\partial \Sigma}^{\prime}=\int_{\partial \Sigma} d x^{0}\left\{A_{j}^{X} \partial_{0}^{A} X^{j}+A_{\bar{\jmath}}^{X} \partial_{0}^{A} \bar{X}^{\bar{\jmath}}\right\}
$$

and gauge invariance follows since (4.32) is obeyed, which implies that the line bundle $\mathcal{O}_{\mathbb{C} P^{N-1}}(-n)$ supported by the equivariant $\mathrm{B}$-brane has $U(1)^{N-1}$-equivariant structure.

The boundary conditions for the GNLSM with the $\mathbb{C} P^{N-1}$ target can similarly be found; for the $U(1)^{N-1}$ vector multiplets, the boundary conditions follow from (4.47) and (4.48), while for the matter fields, the boundary conditions are

$$
\begin{array}{r}
g_{\bar{\jmath} i}\left(\psi_{-}^{Z_{-}^{i}}-\psi_{+}^{Z^{i}}\right)+2 \pi F_{\bar{\jmath} i}^{X}\left(\psi_{+}^{Z^{i}}+\psi_{-}^{Z^{i}}\right)=0, \\
g_{\bar{j} i} \partial_{1}^{A} Z^{i}-2 \pi F_{\bar{j} i}^{X} \partial_{0}^{A} Z^{i}=0,
\end{array}
$$

and their $B$-type supersymmetric completions, where $g$ is the Fubini-Study metric and $F$ is the curvature of $\mathcal{O}_{\mathbb{C} P^{N-1}}(-n)$ given in (4.23).

An alternative formulation also exists for $U(1)^{k_{-}}$ GNLSMs with $X=\mathbb{C}^{N} / / U(1)^{N-k}$, i.e., general Kähler toric manifolds. The boundary action for the $U(1)^{N-k} \times$ $U(1)^{k}$ GLSM is

$$
S_{\partial \Sigma}=\frac{\theta^{\prime}}{4 \pi r^{\prime}} \int_{\partial \Sigma} d x^{0} \sum_{i}^{N}\left(i D_{0} \bar{\phi}_{i} \phi_{i}-i \bar{\phi}_{i} D_{0} \phi_{i}\right)
$$

where $\theta^{\prime}=2 \pi n^{\prime}\left(n^{\prime} \in \mathbb{Z}\right)$ and $r^{\prime} \in \mathbb{R}$, together with the theta terms

$$
\sum_{b}^{N-k}\left(\frac{\hat{\theta}_{b}}{2 \pi} \int_{\Sigma} \hat{F}_{01 b} d^{2} x\right)+\sum_{c}^{k}\left(\frac{\tilde{\theta}_{c}}{2 \pi} \int_{\Sigma} \tilde{F}_{01 c} d^{2} x\right) .
$$

Setting

$$
\frac{\theta^{\prime}}{r^{\prime}}=\frac{\hat{\theta}_{b}}{\hat{r}_{b}}=\frac{\tilde{\theta}_{c}}{\tilde{r}_{c}}
$$

and

$$
\gamma^{\prime}=\hat{\gamma}_{b}=\tilde{\gamma}_{c},
$$

the relevant boundary conditions are (4.46), (4.47), and (4.48), with $\frac{\hat{\theta}}{\hat{r}}$ replaced by $\frac{\theta^{\prime}}{r^{\prime}}$ and $\hat{\gamma}$ replaced by $\gamma^{\prime}$. In addition, the $D_{a}$ equation of motion is also necessary for complete $B$-type supersymmetry at the boundaries.

By taking the $\hat{e}_{b} \rightarrow \infty$ limit and repeating the familiar procedure, we can obtain the GNLSM boundary action that includes the Hermitian connection of the $U(1)^{k}$ equivariant holomorphic line bundle $\otimes_{b=1}^{N-k} \mathcal{O}_{X}\left(-n_{b}\right)$ over $\mathbb{C}^{N} / / U(1)^{N-k}$ given by (4.39), as well as the relevant GNLSM boundary conditions.
An important advantage of the alternative formulation of equivariant B-branes over the first one is that because of the constraints (4.44) and (4.57), the form of the GLSM boundary action does not depend on which gauge symmetries we are breaking to obtain the GNLSM. This implies the equivalence of equivariant B-branes in different toric targets of GNLSMs obtained from a single GLSM. To ensure that the first formulation also does not depend on which gauge symmetries we are breaking, we can impose the same constraints for it.

\section{Quantum corrections}

We have heretofore analyzed the boundary conditions of the classical $U(1)^{N-k} \times U(1)^{k}$ GLSM, and the respective GNLSM limits of these conditions, in two equivalent formulations. We shall now investigate quantum effects for the alternative formulation of equivariant B-branes given in Sec. IV. C, ${ }^{17}$ since we shall use this formulation for the proof of mirror symmetry in the following section. ${ }^{18}$

There are two quantum effects of the $U(1)^{N-k} \times U(1)^{k}$ GLSM with $\sum_{i=1}^{N} \mathcal{Q}_{i a} \neq 0$ that are important. The first of these is the running of the FI parameters

$$
r_{0 a}=r_{a}(\mu)+\sum_{i=1}^{N} \mathcal{Q}_{i a} \log \left(\frac{\Lambda_{\mathrm{UV}}}{\mu}\right)
$$

where $r_{0 a}$ denotes bare parameters, $\Lambda_{\mathrm{UV}}$ is an ultraviolet cutoff, and $\mu$ is a finite energy scale. By integrating the beta functions of the FI parameters, $\beta_{a}=\mu \frac{d r_{a}}{d \mu}$, the $\mu$ dependence is found to be

$$
r_{a}(\mu)=\sum_{i=1}^{N} \mathcal{Q}_{i a} \log \left(\frac{\mu}{\Lambda}\right)
$$

where $\Lambda$ is the renormalization group invariant dynamical scale. The running of $r_{a}$ implies that the phase, $e^{i \gamma_{a}}=$ $t_{a} /\left|t_{a}\right|$, which appears in the boundary conditions we have used, changes with the renormalization group flow. The second quantum effect is the anomaly of the $U(1)$ axial $R$ symmetry, whereby axial $R$ rotations $\psi_{ \pm i} \rightarrow e^{ \pm i \beta / 2} \psi_{ \pm i}$, $\sigma_{a} \rightarrow e^{-i \beta} \sigma_{a}$, and $\lambda_{ \pm a} \rightarrow e^{ \pm i \beta / 2} \lambda_{ \pm a}$ no longer leave the action invariant, but result in a shift of the theta angles, i.e.,

$$
\theta_{a} \rightarrow \theta_{a}+\sum_{i=1}^{N} \mathcal{Q}_{i a} \beta .
$$

These effects should be apparent in a quantum effective description, whereby the lowest components $\sigma_{a}$ of the

\footnotetext{
${ }^{17} \mathrm{We}$ shall not study the quantum effects for the first formulation, since the main quantum correction is the running of the FI parameters, and the FI parameters do not enter the boundary conditions in that formalism.

${ }^{18}$ The following is a generalization of the analysis given in Sec. 6 of [9] to the case of multiple $U(1)$ gauge groups.
} 
superfields $\Sigma_{a}$ are chosen to be slowly varying and to be large compared to the energy scale $\mu$ at which we look at the effective theory. This imparts large masses to the charged matter superfields $\Phi_{i}$, which can then be integrated out as long as we are studying the theory at some finite energy scale $\mu$. From a path integral computation [20], the superpotential of the effective action, which corresponds to a Landau-Ginzburg model, ${ }^{19}$ is

$$
\begin{aligned}
\tilde{W}_{\text {(eff) }}= & -\sum_{a=1}^{N}\left[\sum_{i}^{N} \mathcal{Q}_{i a}\left(\log \left(\frac{\sum_{a^{\prime}}^{N} \mathcal{Q}_{i a^{\prime}} \Sigma_{a^{\prime}}}{\mu}\right)-1\right)\right] \Sigma_{a} \\
& -\sum_{a=1}^{N} t_{a}(\mu) \Sigma_{a}
\end{aligned}
$$

where from the effective FI-theta parameter

$$
t_{(\mathrm{eff}) a}=t_{a}(\mu)+\sum_{i}^{N} \mathcal{Q}_{i a}\left(\log \left(\frac{\sum_{a^{\prime}}^{N} \mathcal{Q}_{i a^{\prime}} \Sigma_{a^{\prime}}}{\mu}\right)\right)
$$

is obtained. Now, by performing an ordinary axial $R$ rotation $\Sigma_{a} \rightarrow e^{-i \beta} \Sigma_{a}$ in (4.63), we can retrieve the shift (4.61).

Now, it is known from [9] that a $\mathrm{D}$-brane which preserves the $B$-type supercharges $Q_{B}=\bar{Q}_{+}+\bar{Q}_{-}$and $Q_{B}^{\dagger}=Q_{+}+$ $Q_{-}$is a Lagrangian submanifold of the space $\mathbb{C}^{N}$ defined by the fields $\sigma_{a}$. In addition, this D-brane ought to be the preimage of a horizontal straight line in the $\tilde{W}_{\text {(eff) }}$ plane, i.e., $\operatorname{Im}\left(\tilde{W}_{\text {(eff) }}(\sigma)\right)=$ const. If we were to solve these constraints in terms of $\sigma_{a}$, then we will obtain the quantum corrected boundary condition for $\sigma_{a}$. In general, these constraints are difficult to solve. However, when the parameters $\theta_{a}=0$, then there is the solution $\sigma_{a}=\left|\sigma_{a}\right|$, which satisfies $\operatorname{Im}\left(\sigma_{a}\right)=0$ and $\operatorname{Im}\left(\tilde{W}_{\text {(eff) }}(\sigma)\right)=0$.

To obtain a less trivial solution, we can perform an axial $R$ rotation, which includes the shift of $\theta_{a}=0$ to $\theta_{a}=\sum_{i=1}^{N} \mathcal{Q}_{i a} \beta$, due to the aforementioned anomaly. Then, we obtain the solution $\sigma_{a}=e^{i \beta}\left|\sigma_{a}\right|$, which satisfies $\operatorname{Im}\left(e^{-i \beta} \sigma_{a}\right)=0$ and the straight line equation $\operatorname{Im}\left(e^{-i \beta} \tilde{W}_{\text {(eff) }}(\sigma)\right)=0$. These conditions are compatible with the constraints of the $B$-type supercharges

$$
Q_{B}=\bar{Q}_{+}+e^{i \beta} \bar{Q}_{-}
$$

and $Q_{B}^{\dagger}=Q_{+}+e^{-i \beta} Q_{-}$found in [9]; i.e., the D-brane ought to be a Lagrangian submanifold of the field space $\mathbb{C}^{N}$, and it ought to be the preimage of a straight line in the $\tilde{W}_{\text {(eff) }}$ plane with slope $\tan (\beta)$, i.e., $\operatorname{Im}\left(e^{-i \beta} \tilde{W}_{(\text {eff })}(\sigma)\right)=$ const.

Hence, we find that there is a family of explicit solutions which include

\footnotetext{
${ }^{19}$ To be precise, the theory involves a gauge field, whose only effect is a vacuum energy [20].
}

$$
\begin{aligned}
\sigma_{a} & =e^{i \beta}\left|\sigma_{a}\right|, \\
e^{i \beta / 2} \lambda_{+a}+e^{-i \beta / 2} \lambda_{-a} & =0, \\
e^{-i \beta / 2} \bar{\lambda}_{+a}+e^{i \beta / 2} \bar{\lambda}_{-a} & =0, \quad \text { at } \partial \Sigma,
\end{aligned}
$$

parametrized by $\beta=\theta_{a} / \sum_{i=1}^{N} \mathcal{Q}_{i a},{ }^{20}$ which preserve the $B$-type supercharges $Q_{B}=\bar{Q}_{+}+e^{i \beta} \bar{Q}_{-}$and $Q_{B}^{\dagger}=Q_{+}+$ $e^{-i \beta} Q_{-}$. Other solutions, including those with $\beta \neq$ $\theta_{a} / \sum_{i=1}^{N} \mathcal{Q}_{i a}$, should exist, but in these cases the quantum corrections are nontrivial, and therefore they are difficult to determine, and we shall not consider them.

Now, note that we have $\beta=\theta_{a} / \sum_{i=1}^{N} \mathcal{Q}_{i a}$ for all $a=1, \ldots, N$. Using (4.59) and (4.60), we have $r_{0}=\sum_{i=1}^{N} \mathcal{Q}_{i a} \log \left(\frac{\Lambda_{\mathrm{UV}}}{\Lambda}\right)$, which implies

$$
\frac{\theta_{a}}{r_{0 a}}=\frac{\sum_{i=1}^{N} \mathcal{Q}_{i a} \beta}{\sum_{i=1}^{N} \mathcal{Q}_{i a} \log \left(\frac{\Lambda_{\mathrm{UV}}}{\Lambda}\right)}=\frac{\beta}{\log \left(\frac{\Lambda_{\mathrm{UV}}}{\Lambda}\right)} ;
$$

i.e., we find that $\theta_{a} / r_{0 a}$ are equal for all values of $a .^{21}$ This agrees with the constraints (4.44) and (4.57). In other words, we find that these constraints, which we previously imposed by hand at the classical level, emerge naturally as a result of quantum effects.

\section{E. Mirrors of equivariant B-branes}

In this section, we shall use the alternative formulation for equivariant B-branes, given in Sec. IV. C, to find the Landau-Ginzburg mirrors of equivariant B-branes, following the exposition in Sec. III, as well as the results of [9]. We shall assume in the following that

$$
b_{1 a}=\sum_{i}^{N} \mathcal{Q}_{i a}>0
$$

In particular, $\hat{b}_{1 b}=\sum_{i}^{N} \hat{Q}_{i b}>0$ implies that we are studying the mirrors of GNLSMs with Fano target spaces.

Let us start with the mirrors of equivariant B-branes on Fano manifolds of the form $X=\mathbb{C}^{N} / / U(1)$. We focus on the family of boundary conditions (4.65). The corresponding boundary conditions of the matter fields include

$$
\begin{aligned}
\cos \left(\hat{\gamma}_{0}\right) D_{1} \phi_{i}-i \sin \left(\hat{\gamma}_{0}\right) D_{0} \phi_{i} & =0, \\
e^{-i \hat{\gamma}_{0}+i \beta / 2} \psi_{+i} & =e^{i \hat{\gamma}_{0}-i \beta / 2} \psi_{-i}, \\
e^{i \hat{\gamma}_{0}-i \beta / 2} \bar{\psi}_{+i} & =e^{-i \hat{\gamma}_{0}+i \beta / 2} \bar{\psi}_{-i},
\end{aligned}
$$

where the axial $R$ rotations on the fermionic fields have been taken into account. These boundary conditions preserve the $B$-type supercharge $Q_{B}=\bar{Q}_{+}+e^{i \beta} \bar{Q}_{-}$and

\footnotetext{
${ }^{20} \sigma_{a}=e^{i \beta}\left|\sigma_{a}\right|$ implies the boundary condition $\operatorname{Im}\left(e^{-i \beta} \sigma_{a}\right)=0$.

${ }^{21}$ Naively, it may seem that the boundary action (4.41) vanishes in the continuum limit $\left(\Lambda_{\mathrm{UV}} \rightarrow \infty\right)$ due to (4.66). However, this is not the case, at least for $\sum_{i=1}^{N} \mathcal{Q}_{i a}>0$, as we shall see in the next section.
} 
its conjugate. Now, in the continuum limit $\Lambda_{\mathrm{UV}} \rightarrow \infty$ whereby $\hat{r}_{0}=\hat{b}_{1} \log \left(\Lambda_{\mathrm{UV}} / \Lambda\right) \rightarrow \infty$, we have $\hat{\gamma}_{0} \rightarrow 0$. As a result, the mixed Dirichlet-Neumann boundary conditions on $\phi_{i}$ reduce to pure Neumann boundary conditions.

With these facts in mind, let us shift our attention to the boundary action

$$
\begin{aligned}
S_{\partial \Sigma} & =\frac{\hat{\theta}}{4 \pi \hat{r}_{0}} \int_{\partial \Sigma} \sum_{i=1}^{N}\left(i D_{0} \bar{\phi}_{i} \phi_{i}-i \bar{\phi}_{i} D_{0} \phi_{i}\right) d x^{0} \\
& =\frac{\hat{\theta}}{2 \pi \hat{r}_{0}} \int_{\partial \Sigma} \sum_{i=1}^{N}\left|\phi_{i}\right|^{2}\left(\partial_{0} \varphi_{i}+\sum_{a}^{N} \mathcal{Q}_{i a} A_{0 a}\right) d x^{0} .
\end{aligned}
$$

Now, by integrating over the modes of $\phi_{i}$ in the frequency range $\mu \leq|k| \leq \Lambda_{\mathrm{UV}}$ in the path integral, $\left|\phi_{i}\right|^{2}$ is replaced by $\left\langle\left|\phi_{i}\right|^{2}\right\rangle=\log \left(\Lambda_{\mathrm{UV}} / \mu\right)$. Since $\hat{r}_{0} / \hat{b}_{1}=\log \left(\Lambda_{\mathrm{UV}} / \mu\right)+$ $\hat{r} / \hat{b}_{1}$, taking the continuum limit $\Lambda_{\mathrm{UV}} \rightarrow \infty$ gives us $\left|\phi_{i}\right|^{2} \approx \hat{r}_{0} / \hat{b}_{1}$, which implies that

$$
S_{\partial \Sigma}=\frac{\hat{\theta}}{2 \pi} \int_{\partial \Sigma}\left(\frac{1}{\hat{b}_{1}} \sum_{i=1}^{N} \partial_{0} \varphi_{i}+\hat{A}_{0}+\sum_{c}^{N-1} \frac{\tilde{b}_{1 c}}{\hat{b}_{1}} \tilde{A}_{0 c}\right) d x^{0}
$$

The relevant portion of the action with regard to the dualization of mirror symmetry is then

$$
S_{\varphi}=\frac{1}{2 \pi} \int_{\Sigma} \sum_{i=1}^{N} \frac{\hat{r}_{0}}{\hat{b}_{1}}\left|d \varphi_{i}+\sum_{a}^{N} \mathcal{Q}_{i a} A_{a}\right|^{2}-\frac{i \hat{\theta}}{2 \pi} \int_{\partial \Sigma}\left(\frac{1}{\hat{b}_{1}} \sum_{i=1}^{N} d \varphi_{i}+\hat{A}+\sum_{c}^{N-1} \frac{\tilde{b}_{1 c}}{\hat{b}_{1}} \tilde{A}_{c}\right),
$$

where we have considered the Euclidean signature on the world sheet for simplicity, ${ }^{22}$ and where the terms with fermionic fields that are not essential in the present analysis have been ignored. Let us consider another action with one-form fields $\mathcal{B}_{i}=\mathcal{B}_{i \mu} d x^{\mu}$ given by

$$
S^{\prime}=\sum_{i=1}^{N}\left[\frac{\hat{b}_{1}}{8 \pi \hat{r}_{0}} \int_{\Sigma} \mathcal{B}_{i} \wedge * \mathcal{B}_{i}+\frac{i}{2 \pi} \int_{\Sigma} \mathcal{B}_{i} \wedge\left(d \varphi_{i}+\sum_{a}^{N} \mathcal{Q}_{i a} A_{a}\right)\right]-\frac{i \hat{\theta}}{2 \pi} \int_{\partial \Sigma}\left(\frac{1}{\hat{b}_{1}} \sum_{i=1}^{N} d \varphi_{i}+\hat{A}+\sum_{c}^{N-1} \frac{\tilde{b}_{1 c}}{\hat{b}_{1}} \tilde{A}_{c}\right) .
$$

The one-form fields $\mathcal{B}_{i}$ have the boundary condition

$$
\left.\mathcal{B}_{i}\right|_{\partial \Sigma}=0
$$

i.e., their inner products with tangent vectors of the boundaries vanish. If we were to first integrate out $\mathcal{B}_{i}$, the constraint $\mathcal{B}_{i}=i 2\left(\hat{r}_{0} / \hat{b}_{1}\right) *\left(d \varphi_{i}+\sum_{a}^{N} Q_{i a} A_{a}\right)$ is obtained [whereby the boundary condition (4.73) is consistent with the boundary condition $D_{1} \phi_{i}=0$ obtained in the continuum limit] and the original action (4.71) is obtained. Alternatively, if we were to first integrate out $\varphi_{i}$, the constraint

$$
\mathcal{B}_{i}=d \vartheta_{i}
$$

is obtained, where the fields $\vartheta_{i}$ are periodic with period $2 \pi{ }^{23}$ The boundary conditions (4.73) then imply that $\vartheta_{i}$ are constants at the boundaries of the world sheet. The boundary terms containing $\partial_{2}\left(\delta \varphi_{i}\right)$ obtained when integrating out $\varphi_{i}$ cancel if these constants are

$$
\vartheta_{i}=\hat{\theta} / \hat{b}_{1} \quad \text { at } \partial \Sigma,
$$

for all $i$, where $\hat{\theta} / \hat{b}_{1}=\beta=\theta_{a} / b_{1 a}$. Now, using the constraint (4.74) in (4.72), the mirror action

$$
\begin{aligned}
S_{\vartheta}= & \sum_{i=1}^{N} \frac{1}{2 \pi}\left[\frac{\hat{b}_{1}}{4 \hat{r}_{0}} \int_{\Sigma}\left|d \vartheta_{i}\right|^{2}+i \int_{\Sigma} d \vartheta_{i} \wedge\left(\sum_{a}^{N} \mathcal{Q}_{i a} A_{a}\right)\right]-\frac{i \hat{\theta}}{2 \pi} \int_{\partial \Sigma}\left(\hat{A}+\sum_{c}^{N-1} \frac{\tilde{b}_{1 c}}{\hat{b}_{1}} \tilde{A}_{c}\right) \\
= & \sum_{i=1}^{N} \frac{1}{2 \pi}\left[\frac{\hat{b}_{1}}{4 \hat{r}_{0}} \int_{\Sigma}\left|d \vartheta_{i}\right|^{2}-i \int_{\Sigma} \sum_{a}^{N} \mathcal{Q}_{i a} \vartheta_{i} d A_{a}\right] \\
& +\frac{i}{2 \pi} \int_{\partial \Sigma}\left[\left(\sum_{i=1}^{N} \hat{Q}_{i} \vartheta_{i}-\hat{\theta}\right) \hat{A}+\sum_{c}^{N-1}\left(\sum_{i=1}^{N} \tilde{Q}_{i c} \vartheta_{i}-\frac{\tilde{b}_{1 c}}{\hat{b}_{1}} \hat{\theta}\right) \tilde{A}_{c}\right]
\end{aligned}
$$

\footnotetext{
${ }^{22}$ In the following derivation, we use the notation $|A|^{2}=A \wedge \star A$.

${ }^{23}$ For details on why $\vartheta_{i}$ ought to be periodic, see [20], page 250 .
} 
is obtained. Finally, the boundary term in this action vanishes when we use the boundary condition (4.75), and the dualization process ends with only a bulk action.

In particular, the relationship (reviewed in Sec. III) between the fields of the mirror theories, i.e.,

$$
Y_{i}+\bar{Y}_{i}=2 \bar{\Phi}_{i} e^{\sum_{a}^{N} \mathcal{Q}_{i a} V_{a}} \Phi_{i},
$$

holds, and we have the following relationships between superfield components:

$$
\begin{aligned}
y_{i}= & \varrho_{i}-i \vartheta_{i}, \\
& \left\{\begin{array}{l}
\varrho_{i}=\left|\phi_{i}\right|^{2}, \\
\partial_{ \pm} \vartheta_{i}= \pm 2\left(-\left|\phi_{i}\right|^{2}\left(\partial_{ \pm} \varphi_{i}+\sum_{a}^{N} \mathcal{Q}_{i a} A_{ \pm a}\right)+\bar{\psi}_{ \pm i} \psi_{ \pm i}\right),
\end{array}\right. \\
\chi_{i+}= & 2 \bar{\psi}_{+i} \phi_{i}, \quad \chi_{i-}=-2 \bar{\psi}_{-i} \phi_{i}, \\
\bar{\chi}_{+i}= & 2 \bar{\phi}_{i} \psi_{+i}, \quad \bar{\chi}_{-i}=-2 \bar{\phi}_{i} \psi_{-i}, \\
E_{i}= & -2 \bar{\psi}_{-i} \psi_{+i}-2\left|\phi_{i}\right|^{2} \sum_{a} \mathcal{Q}_{i a} \bar{\sigma}_{a},
\end{aligned}
$$

where $\partial_{ \pm}=\partial_{0} \pm \partial_{1}, Y_{i}=y_{i}+\theta^{+} \bar{\chi}_{+i}+\bar{\theta}^{-} \chi_{-i}+\theta^{+} \bar{\theta}^{-} E_{i}$. The relationship between the periodic fields $\vartheta_{i}$ and $\varphi_{i}$ is, in fact, evidence that mirror symmetry of the two theories stems from $\mathrm{T}$ duality on the phase of the charged chiral superfields $\Phi_{i}$, whereby the neutral twisted chiral superfields $Y_{i}$ are periodic, i.e., $Y_{i} \equiv Y_{i}+2 \pi i$ [20].

Furthermore, the Kähler metric of the target space of the mirror Landau-Ginzburg sigma model is given by

$$
d s^{2}=\frac{\hat{b}_{1}}{4 \hat{r}_{0}} \sum_{i=1}^{N}\left(\left(d \varrho_{i}\right)^{2}+\left(d \vartheta_{i}\right)^{2}\right),
$$

which is the flat cylinder metric on $\left(\mathbb{C}^{\times}\right)^{N}$. As in Sec. III, taking the $\hat{e} \rightarrow \infty$ limit allows us to integrate $\hat{\Sigma}$ out of the action and imposes the constraint

$$
\sum_{j}^{N} \hat{Q}_{j} Y_{j}-\hat{t}=0,
$$

giving us the gauged Landau-Ginzburg theory with holomorphic twisted superpotential

$$
\tilde{W}=\sum_{c}^{N-1}\left(\sum_{j=1}^{N} \tilde{Q}_{j c} Y_{j}-\tilde{t}_{c}\right) \tilde{\Sigma}_{c}+\sum_{j=1}^{N} e^{-Y_{j}} .
$$

We recall that the constraint (4.80) fixes the target space of the gauged Landau-Ginzburg theory to be the algebraic torus $\left(\mathbb{C}^{\times}\right)^{N-1}$.

The boundary conditions (4.75) imply that $e^{-y_{i}}$ have a common phase that is fixed. In other words, the boundaries of the world sheet are mapped by $e^{-y_{i}}$ to a cycle $\gamma_{\hat{\theta}}$ in $\left(\mathbb{C}^{\times}\right)^{N-1}$ which has $N-1$ real dimensions. This cycle is given by

$$
\left(e^{-y_{1}}, \ldots, e^{-y_{N}}\right)=\left(e^{-Q_{1}+i \hat{\theta} / b_{1}}, \ldots, e^{-Q_{N}+i \hat{\theta} / b_{1}}\right),
$$

where $\varrho_{i}$ are constrained by $\sum_{i=1}^{N} \hat{Q}_{i} Q_{i}=\hat{r}$. In the continuum limit, the pure Neumann boundary condition we obtain for $\phi_{i}$ from (4.68), implies the Neumann boundary condition

$$
\partial_{1} \varrho_{i}=0
$$

for the coordinates $\varrho_{i}$ tangent to $\gamma_{\hat{\theta}}$. Using (4.78) and (4.68), we may also obtain boundary conditions on the fermionic dual fields, which are

$$
\begin{aligned}
& e^{-i \beta / 2} \chi_{+i}+e^{i \beta / 2} \chi_{-i}=0, \\
& e^{i \beta / 2} \bar{\chi}_{+i}+e^{-i \beta / 2} \bar{\chi}_{-i}=0 .
\end{aligned}
$$

These boundary conditions correspond to a D-brane wrapped on the cycle $\gamma_{\hat{\theta}}$.

The cycle $\gamma_{\hat{\theta}}$ is a Lagrangian submanifold of $\left(\mathbb{C}^{\times}\right)^{N-1}$. The A-brane wrapping this Lagrangian submanifold is the mirror of the space-filling B-brane supporting the holomorphic line bundle $\mathcal{O}_{X}(-n)$ with $U(1)^{N-1}$-equivariant structure, where $X$ is a Fano toric manifold of the form $\mathbb{C}^{N} / / U(1)$.

Let us investigate this A-brane further, by studying the image of the cycle $\gamma_{\hat{\theta}}$ in the $\tilde{W}$ plane. In particular, we would like to find the mirror of the $U(1)^{N-1}$-equivariant structure on the B-brane. The twisted superpotential (4.81) can be rewritten as

$$
\tilde{W}=\tilde{W}_{\text {equiv }}+\tilde{W}_{X}
$$

where the first and second terms of (4.81) correspond, respectively, to the first and second terms of (4.85). The image of $\gamma_{\hat{\theta}}$ in the $\tilde{W}_{X}$ plane is

$$
\left.\tilde{W}_{X}\right|_{\partial \Sigma}=e^{i \beta} \sum_{i=1}^{N}\left|e^{-y_{i}}\right|,
$$

which is the mirror condition found in [9] when studying the mirrors of B-branes without equivariant structure. In particular, it is a straight line that makes an angle $\beta=\hat{\theta} / \hat{b}_{1}$ with respect to the real axis. Since we have set $\hat{\theta}=2 \pi n$ earlier, and $\int_{X} c_{1}\left(\mathcal{O}_{X}(-n)\right)=-n$, the slope of this straight line depends on the first Chern class of the holomorphic line bundle $\mathcal{O}_{X}(-n)$ supported by the B-brane.

Shifting our focus to the boundary value of $\tilde{W}_{\text {equiv }}$, we find that it is given by

$$
\begin{aligned}
\left.\tilde{W}_{\text {equiv }}\right|_{\partial \Sigma} & =\sum_{c}^{N-1} \operatorname{Re}\left(e^{-i \beta} \tilde{\sigma}_{c}\right) e^{i \beta}\left(\sum_{j}^{N} \tilde{Q}_{j c} Q_{j}-\tilde{r}_{c}\right) \\
& =\sum_{c}^{N-1} \operatorname{Re}\left(e^{-i \beta} \tilde{\sigma}_{c}\right) E q_{c}(\varrho),
\end{aligned}
$$


where we have used the boundary conditions $\vartheta_{i}=\hat{\theta} /$ $\hat{b}_{1}=\beta$ and $\operatorname{Im}\left(e^{-i \beta} \tilde{\sigma}_{c}\right)=0$ as well as the identity $\beta=\tilde{\theta}_{c} /$ $\sum_{i=1}^{N} \tilde{Q}_{i c}$. Here, $E q_{c}$ is a complex-valued map

$$
E q: \gamma_{\hat{\theta}} \rightarrow \mathfrak{t}(1)^{N-1}
$$

where $\mathfrak{u}(1)^{N-1}$ is the Lie algebra of $U(1)^{N-1}$. In particular, for a given value of $c, \gamma_{\theta}$ is mapped to a straight line in the $E q_{c}$ plane, which makes an angle $\beta$ with respect to the real axis. Thus, this map $E q$ from the cycle $\gamma_{\hat{\theta}}$ (on which the A-brane is wrapped) to $\mathfrak{t}(1)^{N-1}$ is the mirror of $U(1)^{N-1}$ equivariant structure on the B-brane. In addition, we note that the boundary value of the total twisted superpotential is

$\left.\tilde{W}\right|_{\partial \Sigma}=e^{i \beta}\left(\sum_{i=1}^{N} e^{-\varrho_{i}}+\sum_{c}^{N-1} \operatorname{Re}\left(e^{-i \beta} \tilde{\sigma}_{c}\right)\left(\sum_{j}^{N} \tilde{Q}_{j c} \varrho_{j}-\tilde{r}_{c}\right)\right)$,

which is a map from $\operatorname{Re}\left(e^{-i \beta} \tilde{\sigma}_{c}\right)$ and $\varrho_{i}$ to a straight line in the $\tilde{W}$ plane which makes an angle $\beta$ with respect to the real axis.

The mirrors of equivariant B-branes on Fano toric manifolds of the form $X=\mathbb{C}^{N} / / U(1)^{N-k}$ can similarly be found using the above method. These mirror A-branes correspond to Lagrangian submanifolds $\left(\gamma_{\theta^{\prime}}\right)$ of the cylinder $\left(\mathbb{C}^{\times}\right)^{k}$, which is defined by

$$
\sum_{j}^{N} \hat{Q}_{j b} Y_{j}-\hat{t}_{b}=0
$$

with the additional data of the superpotential

$$
\tilde{W}=\sum_{c}^{k}\left(\sum_{j=1}^{N} \tilde{Q}_{j c} Y_{j}-\tilde{t}_{c}\right) \tilde{\Sigma}_{c}+\sum_{j=1}^{N} e^{-Y_{j}} .
$$

The first term on the right-hand side of (4.91), when restricted to its boundary value, contains the mirror data of equivariant structure on $\otimes_{b=1}^{N-k} \mathcal{O}_{X}\left(-\hat{n}_{b}\right)$ (which is supported by the space-filling B-brane), which is a map

$$
E q: \gamma_{\theta^{\prime}} \rightarrow \mathfrak{u t}(1)^{k} .
$$

\section{F. Non-Abelian equivariant B-branes}

In this subsection, we shall use the insights obtained from studying Abelian equivariant B-branes to find the description of non-Abelian equivariant B-branes. This will be achieved by generalizing the first formulation studied in this section for Abelian gauge groups (cf. Secs. 4. A-4. B) to non-Abelian gauge groups. Note that the GNLSM notation of Sec. II is used in this subsection. For simplicity, we shall consider only the case where the $B$ field, $C$ field, and $\theta$ parameter of the GNLSM given in (2.5) and (2.6) are zero.

We shall first investigate the boundary conditions required for $B$-type supersymmetry, before proceeding to discuss the admissible boundary action. Now, note that all the terms in $\delta\left(S_{\text {gauge }}+S_{r}\right)$ [Eq. (2.17)] vanish using the following boundary conditions:

$$
\begin{aligned}
\operatorname{Im}\left(\sigma_{a}\right) & =0, \\
\lambda_{+a}+\lambda_{-a} & =0, \\
\partial_{1} \operatorname{Re}\left(\sigma_{a}\right) & =0, \\
A_{1 a} & =0, \\
\partial_{1} A_{0 a} & =0, \\
\partial_{1}\left(\lambda_{-a}-\lambda_{+a}\right) & =0, \\
\partial_{1}\left(D_{a}+\partial_{1} \operatorname{Im}\left(\sigma_{a}\right)\right) & =0 .
\end{aligned}
$$

These conditions are a generalization of the conditions given in (4.20) for the example of $\mathbb{C} P^{N-1}$, except that the boundary condition for $F_{01 a}$ is replaced by the stricter conditions $A_{1 a}=0$ and $\partial_{1} A_{0 a}=0$, and the boundary condition for $\operatorname{Re}\left(\sigma_{a}\right)$ becomes $\partial_{1} \operatorname{Re}\left(\sigma_{a}\right)=0$. These stricter conditions are necessary since we now require that the boundary conditions preserve the locality of the relevant equations of motion when no additional boundary action is added, and because the supersymmetry transformations now contain non-Abelian terms, which causes $B$-type supersymmetry invariance of the set of boundary conditions to not hold unless we use the stricter conditions on the gauge fields. ${ }^{24}$ The boundary conditions, in fact, imply that gauge transformations have to be restricted such that the transformation parameter $\alpha^{a}$ has a vanishing derivative with respect to $x^{1}$ at the boundaries, in order for these boundary conditions to be gauge invariant.

Next, we turn to the boundary conditions for the matter fields. Let us first consider the $\mathcal{N}=1$ subalgebra of $B$-type supersymmetry, which corresponds to $\epsilon_{+}=i \tilde{\epsilon}, \bar{\epsilon}_{+}=-i \tilde{\epsilon}$, $\epsilon_{-}=-i \tilde{\epsilon}$, and $\bar{\epsilon}_{-}=i \tilde{\epsilon}$, where $\tilde{\epsilon}$ is a real parameter. In this case, after integrating out the auxiliary fields $F^{i}$ and $\bar{F}^{\bar{l}}$, we find that (2.16) is

$$
\begin{aligned}
\delta S_{\text {matter }} & \\
= & -\frac{1}{2 \pi} \frac{i \tilde{\epsilon}}{2} \int_{\partial \Sigma} d x^{0}\left\{g_{I J} \partial_{0}^{A} \phi^{I}\left(\psi_{-}^{J}-\psi_{+}^{J}\right)+g_{I J} \partial_{1}^{A} \phi^{I}\left(\psi_{-}^{J}+\psi_{+}^{J}\right)\right. \\
& \left.+g_{I J}\left(\psi_{+}^{I}-\psi_{-}^{I}\right) \operatorname{Re}\left(\sigma^{a}\right) \tilde{e}_{a}^{J}+\omega_{I J}\left(\psi_{+}^{I}+\psi_{-}^{I}\right) \operatorname{Im}\left(\sigma^{a}\right) \tilde{e}_{a}^{J}\right\},
\end{aligned}
$$

\footnotetext{
${ }^{24}$ If we relax the requirement of locality of equations of motion, then the boundary conditions on $A_{0 a}$ and $\operatorname{Re}\left(\sigma_{a}\right)$ become $\partial_{1} A_{0 a}=\tau_{a}$ and $\partial_{1} \operatorname{Re}\left(\sigma_{a}\right)=\tau_{a}$, where $\tau$ is a constant valued in the center of $\mathfrak{g}$.
} 
where $g_{I J} X^{I} Y^{J}=g_{i \bar{j}}\left(X^{i} Y^{\bar{j}}+X^{\bar{j}} Y^{i}\right) \quad$ and $\quad \omega_{I J} X^{I} Y^{J}=$ $i g_{i \bar{\jmath}}\left(X^{i} Y^{\bar{\jmath}}-X^{\bar{\jmath}} Y^{i}\right)$, and where $(I, J, K, \ldots)$ are indices corresponding to real coordinates on $X$. In addition, if we insist on locality of the matter equations of motion, we require that

$$
\begin{aligned}
g_{I J} \delta \phi^{I} \partial_{1}^{A} \phi^{J} & =0, \\
g_{I J}\left(\psi_{-}^{I} \delta_{\nabla} \psi_{-}^{J}-\psi_{+}^{I} \delta_{\nabla} \psi_{+}^{J}\right) & =0
\end{aligned}
$$

at the boundaries, where $\delta_{\nabla} \psi^{J}=\delta \psi^{J}+\Gamma_{K L}^{J} \delta \phi^{K} \psi^{L}$. An equivariant B-brane shall wrap a submanifold (denoted as $\gamma)$ of $X$, to which a boundary of the world sheet is mapped via $\left(\phi^{i}, \bar{\phi}^{\bar{i}}\right)$. Now, any allowed variation of $\phi$ (denoted $\delta \phi^{I}$ for the real coordinate $\phi^{I}$ ) along the boundary, and the derivative along the boundary, $\partial_{0} \phi^{I}$, ought to be tangent to $\gamma$. The first constraint of (4.95) then implies that $\partial_{1} \phi^{J}$ is normal to $\gamma$, since $A_{1 a}=0$ at the boundaries. Then, taking into account the facts that $\operatorname{Im}\left(\sigma_{a}\right)=0$ and $A_{1 a}=0$ at the boundaries, we find that (4.94) vanishes if $\psi_{-}^{I}-\psi_{+}^{I}$ and $\psi_{-}^{I}+\psi_{+}^{I}$ are, respectively, normal and tangent to $\gamma$, and $\tilde{e}_{a}^{I}$ is tangent to $\gamma$, which implies that $\gamma$ is $G$ invariant. In addition, we note that $\psi_{-}^{I}-\psi_{+}^{I}$ being normal to $\gamma$ and $\psi_{-}^{I}+$ $\psi_{+}^{I}$ being tangent to $\gamma$ implies that

$$
\begin{array}{ll}
\psi_{-}^{I}-\psi_{+}^{I}=0, & I: \text { tangent to } \gamma \\
\psi_{-}^{I}+\psi_{+}^{I}=0, & I: \text { normal to } \gamma
\end{array}
$$

(for a choice of coordinates that separates the normal and tangent directions), which satisfies the second constraint of (4.95).

Next, the $\mathcal{N}=(2,2)$ supersymmetry transformation of $\phi^{I}$ is

$$
\delta \phi^{I}=i\left(\epsilon_{+2} \psi_{-}^{I}-\epsilon_{+1} J_{K}^{I} \psi_{-}^{K}-\epsilon_{-2} \psi_{+}^{I}+\epsilon_{-1} J_{K}^{I} \psi_{+}^{K}\right),
$$

where $\epsilon_{+}=\epsilon_{+1}+i \epsilon_{+2}$ and $\epsilon_{-}=\epsilon_{-1}+i \epsilon_{-2}$, and where $J$ is the almost complex structure of $X$ locally given by $J_{k}^{i}=$ $i \delta_{k}^{i}$ and $J_{\bar{k}}^{\bar{i}}=-i \delta_{\bar{k}}^{\bar{i}}$. $B$-type supersymmetry corresponds to $\epsilon_{+1}=-\epsilon_{-1}$ and $\epsilon_{+2}=-\epsilon_{-2}$, whereby

$$
\delta \phi^{I}=i\left(\epsilon_{+2}\left(\psi_{-}^{I}+\psi_{+}^{I}\right)-\epsilon_{+1} J_{K}^{I}\left(\psi_{-}^{K}+\psi_{+}^{K}\right)\right) .
$$

Hence, $\psi_{-}^{I}+\psi_{+}^{I}$ and $J_{K}^{I}\left(\psi_{-}^{K}+\psi_{+}^{K}\right)$ are tangent to $\gamma$, which implies that the application of the almost complex structure, $J$, preserves the tangent space of $\gamma$. Therefore, $\gamma$ is a holomorphically embedded complex submanifold of $X$. This complex submanifold also happens to be $G$ invariant, which we know from the previous paragraph.

Indeed, (2.16) vanishes under this boundary condition; integrating out the auxiliary fields $F^{i}$ and $\bar{F}^{\bar{\imath}},(2.16)$ can be rewritten (for $\epsilon_{+}=-\epsilon_{-}=\epsilon$ ) as

$$
\begin{aligned}
\delta S_{\text {matter }}= & \frac{1}{2 \pi} \frac{1}{4} \int_{\partial \Sigma} d x^{0}\left\{\epsilon \left(-g\left(\partial_{0}^{A} \phi, \psi_{-}-\psi_{+}\right)-i \omega\left(\partial_{0}^{A} \phi, \psi_{-}-\psi_{+}\right)\right.\right. \\
& -g\left(\partial_{1}^{A} \phi, \psi_{-}+\psi_{+}\right)-i \omega\left(\partial_{1}^{A} \phi, \psi_{-}+\psi_{+}\right) \\
& -\operatorname{Re}\left(\sigma^{a}\right) g\left(\tilde{e}_{a}, \psi_{+}-\psi_{-}\right)-i \operatorname{Re}\left(\sigma^{a}\right) \omega\left(\tilde{e}_{a}, \psi_{+}-\psi_{-}\right) \\
& \left.\left.-i \operatorname{Im}\left(\sigma^{a}\right) g\left(\tilde{e}_{a}, \psi_{+}+\psi_{-}\right)+\operatorname{Im}\left(\sigma^{a}\right) \omega\left(\tilde{e}_{a}, \psi_{+}+\psi_{-}\right)\right)+ \text {c.c. }\right\}
\end{aligned}
$$

[where $g(X, Y)=g_{I J} X^{I} Y^{J}$ and $\omega(X, Y)=\omega_{I J} X^{I} Y^{J}$ ], which vanishes using $\operatorname{Im}\left(\sigma_{a}\right)=0$ and $A_{1 a}=0$ as well as the conditions that $\partial_{0} \phi^{I}, \psi_{-}^{I}+\psi_{+}^{I}$, and $\tilde{e}_{a}^{I}$ are tangent to $\gamma$ while $\partial_{1} \phi^{I}$ and $\psi_{-}^{I}-\psi_{+}^{I}$ are normal to $\gamma^{25}$

We may add the $B$-type supersymmetric boundary action

$$
\begin{aligned}
S_{\partial \Sigma}{ }^{\prime} & =\int_{\partial \Sigma} d x^{0}\left\{A_{m}^{X} \partial_{0}^{A} \phi^{m}+A_{\bar{m}}^{X} \partial_{0}^{A} \bar{\phi}^{\bar{m}}+\tilde{R}_{a} \frac{\left(\sigma^{a}+\bar{\sigma}^{a}\right)}{2}-\frac{i}{2} F_{m \bar{n}}^{X}\left(\psi_{+}^{m}+\psi_{-}^{m}\right)\left(\bar{\psi}_{+}^{\bar{n}}+\bar{\psi}_{-}^{\bar{n}}\right)\right\} \\
& =\int_{\partial \Sigma} d x^{0}\left\{A_{m}^{X} \partial_{0} \phi^{m}+A_{\bar{m}}^{X} \partial_{0} \bar{\phi}^{\bar{m}}-i \tilde{R}_{a} \mathcal{A}^{a}-\frac{i}{2} F_{m \bar{n}}^{X}\left(\psi_{+}^{m}+\psi_{-}^{m}\right)\left(\bar{\psi}_{+}^{\bar{n}}+\bar{\psi}_{-}^{\bar{n}}\right)\right\},
\end{aligned}
$$

where we use $(m, \bar{m}, n, \bar{n})$ as coordinate indices on the B-branes, where the curvature of $A^{X}$ satisfies $F_{m n}^{X}=F_{\bar{m}}^{X}=0$, and where $\mathcal{A}_{a}=-i\left(A_{0 a}-\frac{\left(\sigma_{a}+\bar{\sigma}_{a}\right)}{2}\right)$ and

$$
\begin{aligned}
\tilde{R}_{a} & =-A_{m}^{X} \tilde{e}_{a}^{m}-A_{\bar{m}}^{X} \overline{\tilde{e}}_{a}^{\bar{m}} \\
& =-l_{\tilde{e}_{a}} A^{X}
\end{aligned}
$$

\footnotetext{
${ }^{25}$ Recall that for a tangent vector, $T$, and normal vector, $N$, of a holomorphically embedded complex submanifold, $\gamma$, of the Kähler manifold $X$, we have $\omega(T, N)=g(J T, N)=0$.
} 
$B$-type supersymmetry invariance and gauge invariance of this action require the equivariant Bianchi identity

$$
d \tilde{R}=l_{\tilde{e}} F^{X},
$$

and this implies that each B-brane supports a $G$-equivariant holomorphic line bundle (cf. footnote 12 ), for which $\tilde{R}_{a}$ is the moment. ${ }^{26}$ The inclusion of this boundary action results in some of the equations of motion being modified by boundary terms. One may generalize this even further, as shown by Kapustin et al. [11] (cf. Sec. IV. A), by instead including a Wilson line that represents a $G$-equivariant graded holomorphic vector bundle.

In conclusion, we find that in general,

Equivariant $B$-branes are $G$-invariant holomorphically embedded complex submanifolds of $X$, which
support $G$-equivariant holomorphic vector bundles (which may be graded).

As discussed in Sec. IV. A, at least in some cases, this implies that they are objects in the bounded, derived category of $G_{\mathbb{C}}$-equivariant coherent sheaves on $X$.

\section{EQUIVARIANT A-BRANES AND THEIR MIRRORS}

In this section, we study the $A$-type supersymmetric boundary actions and boundary conditions in Abelian GNLSMs on $I \times \mathbb{R}$ with toric target spaces, $X$, as well as their mirror descriptions. These boundary actions and boundary conditions correspond to equivariant A-branes wrapping submanifolds of $X$. Then, with the insights we find from analyzing these abelian equivariant A-branes, we shall proceed to study equivariant A-branes for nonAbelian GNLSMs.

$A$-type supersymmetry is defined by the combination of supercharges

$$
\begin{aligned}
& Q_{A}=\bar{Q}_{+}+e^{i \beta} Q_{-}, \\
& Q_{A}^{\dagger}=Q_{+}+e^{-i \beta} \bar{Q}_{-},
\end{aligned}
$$

where $\beta \in \mathbb{R}$. In what follows, we shall set $\beta=0$ for simplicity, though it is straightforward to study the $\beta \neq 0$ generalization using the same techniques. From (2.15), it can be seen that the corresponding relations among the supersymmetry transformation parameters are

$$
\begin{aligned}
& \epsilon=\epsilon_{+}=\bar{\epsilon}_{-}, \\
& \bar{\epsilon}=\bar{\epsilon}_{+}=\epsilon_{-} .
\end{aligned}
$$

We shall also make use of superfields when discussing boundary conditions and boundary actions, and to this end, we shall make use of the concept of boundaries in superspace [10]. For $A$-type supersymmetry, the relevant boundary in superspace is known as " $A$ boundary" 27 ; and corresponds to

$$
\begin{aligned}
& \theta=\theta^{+}=-\bar{\theta}^{-}, \\
& \bar{\theta}=\bar{\theta}^{+}=-\theta^{-} .
\end{aligned}
$$

Let us first briefly review what is known of ordinary A-branes. For $\mathcal{N}=(2,2)$ NLSMs, the boundary condition needed to preserve $A$-type supersymmetry at the boundaries maps each boundary to a middle-dimensional Lagrangian submanifold of the target space [9]. In addition, we may include the following boundary action:

$$
S_{\partial \Sigma}=\int_{\partial \Sigma} d x^{0} A_{M}^{X} \partial_{0} X^{M}=\int_{x^{1}=\pi} d x^{0} \partial_{0} \phi^{M_{b}} A_{M_{b}}^{X(b)}-\int_{x^{1}=0} d x^{0} \partial_{0} \phi^{M_{a}} A_{M_{a}}^{X(a)},
$$

where $A^{X(a)}$ and $A^{X(b)}$ are the connections of $U(1)$ line bundles on the A-branes $\gamma_{a}$ and $\gamma_{b}$ on which the boundaries $x_{1}=0$ and $x_{1}=\pi$ end [we shall use $(M, N, \ldots)$ as coordinate indices on the Lagrangian submanifold branes]. This boundary action is $A$-type supersymmetric if $F_{M N}^{X}=$ $\partial_{M} A_{N}^{X}-\partial_{N} A_{M}^{X}=0$, i.e., if the line bundles are flat.

\footnotetext{
${ }^{26}$ Note that gauge invariance of the boundary action requires the use of the identity $\alpha^{b} \mathcal{L}_{\tilde{e}_{b}} \tilde{R}_{a}=[\alpha, \tilde{R}]_{a}$.

${ }^{27}$ We shall extensively use the concept of $A$-boundary superspace, which is reviewed in $[10,17]$.
}

We are interested in the generalizations of Lagrangian boundary conditions and the boundary action (5.4) for GNLSMs. These shall be obtained from a GLSM boundary action, using the methods of Sec. III. No boundary conditions shall be imposed by hand at the GLSM level, but rather they shall be understood as being derived through boundary interactions involving "boundary superfields." The advantage of this formulation is that the geometric parameters of the D-brane enter a "boundary F term," and this aids our understanding of quantum corrections. In the following, we shall attempt to generalize the NLSM 
Lagrangian boundary conditions and boundary action (5.4) to the case of $U(1)^{k}$-GNLSMs with Kähler toric target space.

\section{A. Equivariant A-branes on $\mathbb{C}^{N} / / \boldsymbol{U}(\mathbf{1})$ from GLSM}

We shall first consider toric target spaces of the form $\mathbb{C}^{N} / / U(1)$. The $U(1)^{N}$-GLSM boundary action consists of

$$
S_{1}=\frac{1}{2 \pi} \int_{\partial \Sigma} d x^{0}\left[\frac{1}{2} \sum_{i}^{N} \partial_{1}\left|\phi_{i}\right|^{2}+\frac{i}{2} \sum_{i}^{N}\left(\bar{F}_{i} \phi_{i}-\bar{\phi}_{i} F_{i}\right)+\sum_{a}^{N} \frac{i}{4 e_{a}^{2}}\left(\lambda_{-a} \lambda_{+a}-\bar{\lambda}_{+a} \bar{\lambda}_{-a}\right)+\sum_{a}^{N} \theta_{a} A_{0 a}\right]
$$

and

$$
\begin{aligned}
S_{2}= & \frac{1}{2 \pi} \sum_{i}^{N} \int_{\partial \Sigma} d x^{0}\left[\int d \theta d \bar{\theta} \bar{\Phi}_{i} e^{\sum_{a}^{N} \mathcal{Q}_{i a} V_{a}} \Phi_{i}\left(U_{i}-\operatorname{Im} \log \Phi_{i}\right)+\operatorname{Re} \int d \theta s_{i} \Upsilon_{i}\right. \\
& +\sum_{a}^{N} \frac{1}{2 e_{a}^{2}} \int d \theta d \bar{\theta} \operatorname{Re}\left[\Xi_{a}\left(D_{+} \Sigma_{a}-\bar{D}_{-} \Sigma_{a}\right)\right]
\end{aligned}
$$

where $U_{i}$ is a real, bosonic, boundary auxiliary superfield expanded as

$$
U_{i}=u_{i}+\theta \overline{\mathcal{X}}_{i}-\bar{\theta} \mathcal{X}_{i}+\theta \bar{\theta} E_{i}
$$

(with the lowest component $u_{i}$ being a periodic (multivalued) scalar field defined on the boundaries), $\Upsilon_{i}=\bar{D} U$ is the "field strength" of $U_{i}$, expanded as

$$
\Upsilon_{i}:=\bar{D} U_{i}=\mathcal{X}_{i}+\theta\left(E_{i}+i \partial_{0} u_{i}\right)-i \theta \bar{\theta} \partial_{0} \mathcal{X}_{i}
$$

and is a boundary Fermi superfield satisfying $\bar{D} \Upsilon_{i}=0$, while the parameter

$$
s_{i}=c_{i}-i a_{i}
$$

is the boundary analogue of the complex FI-theta parameter. Although both $u_{i}$ and $\varphi_{i}$ are periodic, multivalued functions, the presence of the term $\int d \theta d \bar{\theta} \bar{\Phi}_{i} e^{\sum_{a}^{N} \mathcal{Q}_{i a} V_{a}} \times$ $\Phi_{i}\left(u_{i}-\varphi_{i}\right)$ in (5.6) requires that $u_{i}-\varphi_{i}$ is single valued. We have also introduced an $A$-type supersymmetry invariant boundary $\mathrm{D}$ term for the vector superfields, which contains the complex boundary Fermi superfields

$$
\begin{aligned}
& \Xi_{a}=\xi_{a}+\theta G_{a}+\bar{\theta} H_{a}+\theta \bar{\theta} \mathcal{K}_{a}, \\
& \bar{\Xi}_{a}=\bar{\xi}_{a}+\bar{\theta} \bar{G}_{a}+\theta \bar{H}_{a}+\theta \bar{\theta} \overline{\mathcal{K}}_{a},
\end{aligned}
$$

where $\xi_{a}$ and $\mathcal{K}_{a}$ are fermionic auxiliary fields while $G_{a}$ and $H_{a}$ are bosonic auxiliary fields, all defined along the boundaries. The $A$-type supersymmetry transformations of these fields may be found using the differential operator $\delta=\epsilon \overline{\mathcal{Q}}-\bar{\epsilon} \mathcal{Q}$ defined in [10] on the superfields $\Xi_{a}$ and $\bar{\Xi}_{a}$. In addition, they are defined to be invariant under gauge transformations. The form of (5.6) is chosen such that the boundary conditions

$$
\begin{aligned}
\bar{\Phi}_{i} e^{\sum_{a}^{N} \mathcal{Q}_{i a} V_{a}} \Phi_{i} & =c_{i}, \\
D_{+} \Sigma_{a} & =\overline{D_{\_} \Sigma_{a},} \\
\overline{D_{+} \Sigma_{a}} & =\overline{D_{-} \Sigma_{a}},
\end{aligned}
$$

are effectively imposed via boundary interactions. In components, these are

$$
\begin{aligned}
\left|\phi_{i}\right|^{2} & =c_{i}, \\
\bar{\phi}_{i} \psi_{-i}+\bar{\psi}_{+i} \phi_{i} & =0, \\
\bar{\psi}_{-i} \phi_{i}+\bar{\phi}_{i} \psi_{+i} & =0, \\
i \bar{\phi}_{i} \stackrel{\leftrightarrow}{D}_{1} \phi_{i}+\bar{\psi}_{+i} \psi_{+i}-\bar{\psi}_{-i} \psi_{-i}+\bar{F}_{i} \phi_{i}+\bar{\phi}_{i} F_{i} & =0,
\end{aligned}
$$

and

$$
\begin{aligned}
\lambda_{+a}-\bar{\lambda}_{-a} & =0, \\
\partial_{1} \sigma_{a} & =0, \\
F_{01 a} & =0, \\
D_{a} & =0, \\
\partial_{1}\left(\lambda_{+a}+\bar{\lambda}_{-a}\right) & =0,
\end{aligned}
$$

and the complex conjugates of the conditions in (5.13).

The supersymmetry transformation of the bulk GLSM action together with (5.5) is

$\delta\left(S+S_{1}\right)=\sum_{a}^{N} \frac{r_{a}}{4 \pi} \int_{\partial \Sigma} d x^{0}\left\{\epsilon\left(\bar{\lambda}_{+a}+\lambda_{-a}\right)-\bar{\epsilon}\left(\bar{\lambda}_{-a}+\lambda_{+a}\right)\right\}$.

Now, the $U(1)^{N}$ gauge invariance of the first term in (5.6) requires that $U_{i}$ transforms under $U(1)^{N}$ gauge transformations as 


$$
U_{i} \rightarrow U_{i}+\sum_{a}^{N} \frac{\mathcal{Q}_{i a}}{2}\left(A_{a}+\bar{A}_{a}\right)
$$

in order to cancel the gauge variation of $\operatorname{Im} \log \Phi_{i}$. This implies the following modification of the supersymmetry transformations of the components of $U_{i}$ in order to preserve the Wess-Zumino gauge:

$$
\begin{aligned}
& \delta u_{i}=\epsilon \mathcal{X}_{i}-\bar{\epsilon} \overline{\mathcal{X}}_{i} \\
& \delta \mathcal{X}_{i}=-\bar{\epsilon}\left(E_{i}+i\left(\partial_{0} u_{i}+\sum_{a}^{N} \mathcal{Q}_{i a} A_{0 a}\right)\right)-i \epsilon \sum_{a}^{N} \mathcal{Q}_{i a} \sigma_{a} \\
& \delta \overline{\mathcal{X}}_{i}=-\epsilon\left(E_{i}-i\left(\partial_{0} u_{i}+\sum_{a}^{N} \mathcal{Q}_{i a} A_{0 a}\right)\right)+i \bar{\epsilon} \sum_{a}^{N} \mathcal{Q}_{i a} \bar{\sigma}_{a}, \\
& \delta E_{i}=i \epsilon \partial_{0} \mathcal{X}_{i}+i \bar{\epsilon} \partial_{0} \overline{\mathcal{X}}_{i}-\frac{1}{2} \epsilon \sum_{a}^{N} \mathcal{Q}_{i a}\left(\lambda_{-a}+\bar{\lambda}_{+a}\right)+\frac{1}{2} \bar{\epsilon} \sum_{a}^{N} \mathcal{Q}_{i a}\left(\bar{\lambda}_{-a}+\lambda_{+a}\right) .
\end{aligned}
$$

The boundary superpotential term in (5.6) is not invariant under supersymmetry, ${ }^{28}$ but rather varies as

$$
\delta\left[\frac{1}{2 \pi} \sum_{i}^{N} \int_{\partial \Sigma} d x^{0} \operatorname{Re} \int d \theta s_{i} \Upsilon_{i}\right]=-\frac{\sum_{a}^{N} \sum_{i}^{N} \mathcal{Q}_{i a} c_{i}}{4 \pi} \int_{\partial \Sigma} d x^{0}\left\{\epsilon\left(\bar{\lambda}_{+a}+\lambda_{-a}\right)-\bar{\epsilon}\left(\bar{\lambda}_{-a}+\lambda_{+a}\right)\right\}
$$

Hence, supersymmetry invariance of the entire action requires that (5.14) and (5.17) cancel, which is possible if and only if

$$
\sum_{i}^{N} \mathcal{Q}_{i a} c_{i}=r_{a}
$$

Likewise, the first part (5.5) of the boundary action is not $U(1)^{N}$-gauge invariant, but varies as

$$
\delta S_{1}=\frac{1}{2 \pi} \int_{\partial \Sigma} d x^{0} \sum_{a}^{N} \theta_{a}\left(-\partial_{0} \alpha_{a}\right),
$$

while the boundary superpotential term varies under gauge transformations as

$$
\delta\left[\frac{1}{2 \pi} \sum_{i}^{N} \int_{\partial \Sigma} d x^{0} \operatorname{Re} \int d \theta s_{i} \Upsilon_{i}\right]=\frac{\sum_{a}^{N} \sum_{i}^{N} \mathcal{Q}_{i a} a_{i}}{2 \pi} \int_{\partial \Sigma} d x^{0} \partial_{0} \alpha_{a}
$$

since the residual gauge transformation $A_{a}=\alpha_{a}(x)$ of the Wess-Zumino gauge shifts $u_{i} \rightarrow u_{i}+\sum_{a}^{N} \mathcal{Q}_{i a} \alpha_{a}$, while leaving $\mathcal{X}_{i}$ and $E_{i}$ invariant. Therefore, gauge invariance of the boundary action follows if ${ }^{29}$

$$
\sum_{i}^{N} \mathcal{Q}_{i a} a_{i}=\theta_{a}
$$

Combining (5.18) and (5.21), we find that we need

$$
\sum_{i}^{N} \mathcal{Q}_{i a} s_{i}=t_{a}
$$

for gauge invariance and $A$-type supersymmetry invariance of the action.

\footnotetext{
${ }^{28}$ This nonzero variation occurs because the boundary Fermi superfield $\Upsilon$ is not invariant under the gauge transformation (5.15).

${ }^{29}$ In fact, (5.21) only needs to hold up to the additional term $2 \pi m$, where $m \in \mathbb{Z}$, since the path integral remains gauge invariant in such cases. However, we shall set $m=0$ in the following for simplicity.
} 
Expanding the boundary action in components, we have

$$
\begin{aligned}
S_{\partial \Sigma}= & S_{1}+S_{2} \\
= & \frac{1}{2 \pi} \int_{\partial \Sigma} d x^{0}\left(\sum_{a}^{N} \frac{i}{4 e_{a}^{2}}\left(\lambda_{-a} \lambda_{+a}-\bar{\lambda}_{+a} \bar{\lambda}_{-a}\right)+\sum_{a}^{N} \theta_{a} A_{0 a}\right) \\
& +\frac{1}{2 \pi} \sum_{i}^{N} \int_{\partial \Sigma} d x^{0}\left(\left(i \bar{\phi}_{i} \stackrel{\leftrightarrow}{D}_{1} \phi_{i}+\bar{\psi}_{+i} \psi_{+i}-\bar{\psi}_{-i} \psi_{-i}+\bar{F}_{i} \phi_{i}+\bar{\phi}_{i} F_{i}\right) u_{i}^{\prime}\right. \\
& +\left(\bar{\phi}_{i} \psi_{-i}+\bar{\psi}_{+i} \phi_{i}\right) \overline{\mathcal{X}}_{i}+\mathcal{X}_{i}\left(\bar{\psi}_{-i} \phi_{i}+\bar{\phi}_{i} \psi_{+i}\right)-i \frac{3}{2} \frac{\bar{\phi}_{i}}{\phi_{i}} \psi_{+i} \psi_{-i}+i \frac{3}{2} \frac{\phi_{i}}{\bar{\phi}_{i}} \bar{\psi}_{-i} \bar{\psi}_{+i} \\
& \left.-\left(\left|\phi_{i}\right|^{2}-c_{i}\right) E_{i}+a_{i} \partial_{0} u_{i}\right) \\
& +\sum_{a}^{N} \frac{1}{2 e_{a}^{2}} \int_{\partial \Sigma} d x^{0} \frac{1}{2}\left(\xi_{a}\left(\partial_{0}\left(\lambda_{-a}-\bar{\lambda}_{+a}\right)+2 \partial_{1}\left(\lambda_{-a}+\bar{\lambda}_{+a}\right)\right)\right. \\
& \left.+i 2 G_{a}\left(\partial_{1} \sigma_{a}\right)-2 H_{a}\left(D_{a}-i F_{01 a}\right)+i \mathcal{K}_{a}\left(\lambda_{-a}-\bar{\lambda}_{+a}\right)+\text { c.c. }\right),
\end{aligned}
$$

where the covariant derivative of the scalar fields is given in (3.9). First, we note that Stoke's theorem implies

$$
\frac{a_{i}}{2 \pi} \int_{\partial \Sigma} \partial_{0} u_{i} d x^{0}=\frac{a_{i}}{2 \pi} \int_{\partial \Sigma}\left\{\partial_{0} \varphi_{i}+\partial_{0}\left(u_{i}-\varphi_{i}\right)\right\} d x^{0}=\frac{a_{i}}{2 \pi} \int_{\partial \Sigma} \partial_{0} \varphi_{i} d x^{0},
$$

since $u_{i}-\varphi_{i}$ is single valued. Then, taking the $\hat{e} \rightarrow \infty$ limit, and subsequently integrating out the boundary auxiliary fields, we obtain the boundary action

$$
S_{\partial \Sigma}=\frac{1}{2 \pi} \int_{\partial \Sigma} d x^{0}\left(\sum_{i}^{N} a_{i} \partial_{0} \varphi_{i}+\hat{\theta} \hat{A}_{0}+\sum_{c}^{N-1} \tilde{\theta}_{c} \tilde{A}_{0 c}\right),
$$

together with the boundary conditions (5.12) on the matter fields, as well as boundary conditions

$$
\begin{aligned}
\tilde{\lambda}_{+c}-\overline{\tilde{\lambda}}_{-c}=0, \\
\partial_{1} \tilde{\sigma}_{c}=0, \\
\tilde{F}_{01 c}=0, \\
\tilde{D}_{c}=0, \\
\partial_{1}\left(\tilde{\lambda}_{+c}+\overline{\tilde{\lambda}}_{-c}\right)=0,
\end{aligned}
$$

on vector multiplet fields, and their complex conjugates. Before proceeding, we note that the boundary conditions on the matter fermion fields in (5.12) ensure that the spurious boundary term (3.28) vanishes.

Now, we shall rewrite (5.25) as

$$
\frac{1}{2 \pi} \int_{\partial \Sigma} d x^{0}\left(\sum_{i}^{N} a_{i} \tilde{D}_{0} \varphi_{i}+\hat{\theta} \hat{A}_{0}\right),
$$

where we have used $\sum_{i}^{N} \tilde{Q}_{i c} a_{i}=\tilde{\theta}_{c}$ and where the covariant derivative of $\varphi_{i}$ is

$$
\tilde{D}_{0} \varphi_{i}=\partial_{0} \varphi_{i}+\sum_{c}^{N-1} \tilde{Q}_{i c} \tilde{A}_{0 c}
$$

which agrees with the general definition for scalar fields given in (2.7). By integrating the vector multiplet out of the bulk action [cf. (3.15)], we obtain

$$
\begin{aligned}
\hat{A}_{0} & =\frac{1}{2} \frac{\sum_{i=1}^{N} \hat{Q}_{i}\left(i \bar{\phi}_{i}{\stackrel{\leftrightarrow}{D_{0}}}_{\phi_{i}}+\bar{\psi}_{-i} \psi_{-i}+\bar{\psi}_{+i} \psi_{+i}\right)}{\sum_{j=1}^{N} \hat{Q}_{j}^{2}\left|\phi_{j}\right|^{2}} \\
& =-\frac{\sum_{i=1}^{N} \hat{Q}_{i} c_{i} \tilde{D}_{0} \varphi_{i}}{\sum_{j=1}^{N} \hat{Q}_{j}^{2} c_{j}}
\end{aligned}
$$

at the boundaries, ${ }^{30}$ where (5.12) has been used in the last step. Hence, the final boundary action is ${ }^{31}$

$$
S_{\partial \Sigma}=\frac{1}{2 \pi} \int_{\partial \Sigma} d x^{0}\left[\sum_{i=1}^{N} a_{i} \tilde{D}_{0} \varphi_{i}-\hat{\theta} \frac{\sum_{i=1}^{N} \hat{Q}_{i} c_{i} \tilde{D}_{0} \varphi_{i}}{\sum_{j=1}^{N} \hat{Q}_{j}^{2} c_{j}}\right] .
$$

\footnotetext{
${ }^{30}$ The presence of the boundary term proportional to $\hat{\theta} \hat{A}_{0}$ ensures that the algebraic equation of motion for $\hat{A}_{0}$ does not contain a boundary term; see footnote 3 .

${ }^{31}$ To be precise, the complete boundary action includes the $C$-field term given in (2.5). However, to simplify the following arguments, we shall consider the $C$-field term to be part of the bulk action, by using Stoke's theorem to promote it to a bulk term.
} 
Now, let us investigate the example of $X=\mathbb{C} P^{N-1}$. First, the inhomogeneous coordinates (3.20) that parametrize a local patch of $\mathbb{C} P^{N-1}$ can be written as

$$
\left|Z^{i}\right| e^{i \gamma^{i}}=\frac{\left|\phi_{i}\right| e^{i \varphi_{i}}}{\left|\phi_{N}\right| e^{i \varphi_{N}}}
$$

In other words, the argument of $Z^{i}$ is

$$
\gamma^{i}=\varphi_{i}-\varphi_{N}
$$

We then derive the following A-type supersymmetric boundary conditions of the GNLSM matter fields from (5.12) using the parametrizations (3.19) and $(3.21)^{32}$ :

$\begin{aligned}\left|Z^{i}\right|^{2} & =\frac{c_{i}}{c_{N}} \\ \bar{Z}^{i} \psi_{-}^{Z^{i}}+Z^{i} \bar{\psi}_{+}^{\bar{Z}^{i}} & =0, \\ \bar{Z}^{i} \psi_{+}^{Z^{i}}+Z^{i} \bar{\psi}_{-}^{i} & =0, \\ i\left(\bar{Z}^{i} \partial_{1}^{A} Z^{i}-Z^{i} \partial_{1}^{A} \bar{Z}^{i}\right)+\bar{\psi}_{+}^{\bar{Z}^{i}} \psi_{+}^{Z^{i}}-\bar{\psi}_{-}^{\bar{Z}^{i}} \psi_{-}^{Z^{i}}+F^{Z^{i}} \bar{Z}^{i}+\bar{F}^{\bar{Z}^{i}} Z^{i} & =0,\end{aligned}$

where the last condition is, in fact, a Neumann boundary condition on $\gamma^{i}$, since $\bar{Z}^{i} \partial_{1}^{A} Z^{i}-Z^{i} \partial_{1}^{A} \bar{Z}^{i}=2 \mid Z^{i}{ }^{2} i \partial_{1}^{A} \gamma^{i}$, where

$$
\partial_{\mu}^{A} \gamma^{i}=\partial_{\mu} \gamma^{i}+\sum_{c}^{N-1}\left(\tilde{Q}_{i c}-\tilde{Q}_{N c}\right) \tilde{A}_{\mu c}=\partial_{\mu} \gamma^{i}+\sum_{c}^{N-1} \tilde{e}_{c}^{\gamma^{i}} \tilde{A}_{\mu c}
$$

with $\tilde{e}_{c}^{\gamma^{i}}$ being the Killing vector field that generates the $U(1)^{N-1}$ isometry of the torus, $T^{N-1}$, parametrized by $\gamma_{i}$. The Neumann boundary condition on $\gamma^{i}$ together with the Dirichlet boundary condition on $\left|Z^{i}\right|$ implies that the equivariant A-brane wraps this torus. ${ }^{33}$ Furthermore, this torus is a Lagrangian submanifold of $\mathbb{C} P^{N-1}$ with respect to the Fubini-Study Kähler form given by (4.24). The remaining boundary conditions, i.e., for the fields in the vector multiplet of the GNLSM, are given by (5.26). The complete set of GNLSM boundary conditions is invariant under the $U(1)^{N-1}$ gauge symmetry and satisfies the supersymmetry transformations given in (2.13) and (2.14) for $\epsilon_{+}=\bar{\epsilon}_{-}$. In addition, the boundary conditions also ensure the locality of the classical equations of motion, i.e., that they contain no boundary terms.

Next, with the aid of (5.21), we can rewrite the boundary action (5.30) as

$$
\begin{aligned}
S_{\partial \Sigma} & =\frac{1}{2 \pi} \int_{\partial \Sigma} d x^{0} \sum_{i=1}^{N}\left[a_{i}-\hat{\theta} \frac{c_{i}}{\sum_{j=1}^{N} c_{j}}\right] \tilde{D}_{0} \varphi_{i} \\
& =\frac{1}{2 \pi} \int_{\partial \Sigma} d x^{0}\left[\sum_{i=1}^{N-1}\left(a_{i}-\left(\sum_{k=1}^{N} a_{k}\right) \frac{c_{i}}{\sum_{j=1}^{N} c_{j}}\right) \tilde{D}_{0} \varphi_{i}+\left(a_{N}-\left(\sum_{k=1}^{N} a_{k}\right) \frac{c_{N}}{\sum_{j=1}^{N} c_{j}}\right) \tilde{D}_{0} \varphi_{N}\right] \\
& =\frac{1}{2 \pi} \int_{\partial \Sigma} d x^{0}\left[\sum_{i=1}^{N-1}\left(a_{i}-\left(\sum_{k=1}^{N} a_{k}\right) \frac{c_{i}}{\sum_{j=1}^{N} c_{j}}\right)\left(\partial_{0}\left(\varphi_{i}-\varphi_{N}\right)+\sum_{c}^{N-1}\left(\tilde{Q}_{i c}-\tilde{Q}_{N c}\right) \tilde{A}_{0 c}\right)\right],
\end{aligned}
$$

or

$$
S_{\partial \Sigma}=\int_{\partial \Sigma} d x^{0}\left(\sum_{i=1}^{N-1} A_{i}^{X} \partial_{0} \gamma^{i}-\sum_{c=1}^{N-1} \tilde{R}_{c} \tilde{A}_{0 c}\right)
$$

\footnotetext{
${ }^{32}$ The last condition of (5.12) is actually trivialized using the algebraic equation of motion of $\hat{A}_{1}$ in (3.15). The last condition of (5.33) is obtained via $A$-type supersymmetry transformations of the fermionic boundary conditions.

${ }^{33} \mathrm{Here}$, both boundaries are mapped to the same A-brane. If the boundaries are assigned unique parameters $s_{i}^{\pi}$ and $s_{i}^{0}$ in (5.6), then each boundary is mapped to a different A-brane. However, for simplicity, in most of what follows in this section, we shall assume that both boundaries are assigned the same parameter $s_{i}$.
} 
where $A_{i}^{X}=\frac{1}{2 \pi}\left(a_{i}-\left(\sum_{k=1}^{N} a_{k}\right) \frac{c_{i}}{\sum_{j=1}^{N} c_{j}}\right)$ is the connection of a flat $U(1)$ bundle on the Lagrangian torus $T^{N-1}$ parametrized by $\gamma_{i}$, and where

$$
\begin{aligned}
\tilde{R}_{c} & =-\sum_{i}^{N-1}\left(\tilde{Q}_{i c}-\tilde{Q}_{N c}\right) A_{i}^{X} \\
& =-\sum_{i}^{N-1} \tilde{e}_{c}^{\gamma^{i}} A_{i}^{X} \\
& =-l_{\tilde{e}_{c}} A^{X} .
\end{aligned}
$$

As we explain below, A-type supersymmetry invariance holds since

$$
d \tilde{R}=l_{\tilde{e}} F^{X},
$$

which is equal to zero because $F^{X}=0$. This is known as the equivariant Bianchi identity and implies that the flat $U(1)$ bundle has $U(1)^{N-1}$-equivariant structure, ${ }^{34}$ for which $\tilde{R}_{c}$ is the moment [22,23].

Now, the boundary action (5.36) is not invariant under the supersymmetry transformations (2.13) and (2.14) for $\epsilon_{+}=\bar{\epsilon}_{-}$. Instead, the total action $S+S_{\partial \Sigma}$ is invariant under these transformations at the boundaries, using the boundary conditions (5.33) and (5.26), and therefore the sum of the expressions (2.16), (2.17), and (2.18) with the supersymmetry variation of the boundary action vanishes. The proof of this involves the supersymmetry invariance of the constant moment $\tilde{R}_{c}$, which is essentially the equivariant Bianchi identity (5.38), as well as the boundary constraint

$$
\tilde{\mu}_{c}=-\tilde{r}_{c}
$$

on the moment map, which can be derived from (5.18) using $c_{i}=\left|\phi_{i}\right|^{2}$ and the parametrization (3.19). Furthermore, the first term of the boundary action (5.36) is supersymmetry invariant via Stoke's theorem since the fermionic superpartners of $\gamma^{i}$ are not periodic nor multivalued ([20], page 307), and the nonzero supersymmetry variation of the boundary action is canceled by the $C$ term in (2.18) and the $\tilde{\theta}_{c}$ term in (2.17) via

$$
2 \pi \tilde{R}_{c}=-\tilde{\theta}_{c}+C_{c},
$$

which can be shown to hold via (3.27), (5.39), and (5.22). Finally, the $B$-field terms in (2.18) (where the $B$ field is proportional to the Kähler form) vanish using the boundary conditions given in (5.33).

\footnotetext{
${ }^{34}$ The $G$-equivariant Bianchi identity is equivalent to the $G$ invariance of the connection, $A$, of the bundle $\left(\mathcal{L}_{\tilde{e}} A=0\right)$, which implies that the covariant derivative $d+A$ is $G$ invariant, and this defines a $G$-equivariant bundle; see [22], Sec. 3.2.
}

Next, writing the boundary action as

$$
S_{\partial \Sigma}=\int_{\partial \Sigma} d x^{0}\left(\sum_{i=1}^{N-1} A_{i}^{X} \partial_{0}^{A} \gamma^{i}\right),
$$

it becomes obvious that it is invariant under the gauge transformations given in (2.11) and (2.12), since $A_{i}^{X}$ is a constant and the expression $\partial_{0}^{A} \gamma^{i}$ is invariant under gauge transformations.

We have thus found $A$-type supersymmetric and $U(1)^{N-1}$ gauge invariant boundary conditions and boundary interactions corresponding to an equivariant A-brane in $\mathbb{C} P^{N-1}$, which wraps a Lagrangian submanifold $T^{N-1}$ that supports a $U(1)^{N-1}$-equivariant flat $U(1)$ bundle. We may follow a procedure analogous to that presented above for $\mathbb{C} P^{N-1}$ in order to describe an equivariant A-brane in a toric manifold $X=\mathbb{C}^{N} / / U(1)$ (by choosing different values for $\hat{Q}_{i}$ ), which would again be a Lagrangian submanifold $T^{N-1}$ supporting a flat $U(1)$ bundle with $U(1)^{N-1}$-equivariant structure.

\section{B. Equivariant A-branes on $\mathbb{C}^{N} / / U(1)^{N-k}$ from GLSM}

We can generalize further, since the examples above have been solely for equivariant A-branes on $X=\mathbb{C}^{N} / / U(1)^{N-k}$ where $N-k=1$. For general values of $N-k$, we may derive the relevant boundary conditions and boundary action from the GLSM boundary action (5.23), but instead of taking the $\hat{e} \rightarrow \infty$ limit for a single gauge group, we take $\hat{e}_{b} \rightarrow \infty$, where $b=1, \ldots, N-k$. Integrating out auxiliary fields, and using parametrizations analogous to (3.19) and (3.21), we will be able to derive the $U(1)^{k}$-GNLSM boundary conditions and boundary action that represent an equivariant A-brane wrapping a Lagrangian torus $T^{k}$, which supports a flat $U(1)$ bundle with $U(1)^{k}$-equivariant structure.

Kapustin et al. ([11], page 58) have conjectured that the category of $G$-equivariant A-branes is some sort of $G$-equivariant version of the Fukaya category (which includes Lagrangian submanifolds that support flat unitary vector bundles as objects). Indeed, if we generalize the definition of the equivariant Fukaya category given for finite groups by Cho and Hong ([26], page 68) to $G=U(1)^{k}$, we see that the equivariant A-branes that we have found are objects in the $U(1)^{k}$-equivariant Fukaya category, and therefore, we have partially verified the conjecture of Kapustin et al. The other objects in the category that we have not constructed correspond to Lagrangian submanifolds that support equivariant flat unitary vector bundles.

\section{Quantum corrections}

There are two important quantum effects of the bulk $U(1)^{N-k} \times U(1)^{k}$ GLSM, which affect the FI parameters $r_{a}$ and theta angles $\theta_{a}$ [20]. The first effect is the renormalization of the FI parameters, 


$$
r_{0 a}=r_{a}(\mu)+\sum_{i=1}^{N} \mathcal{Q}_{i a} \log \left(\frac{\Lambda_{\mathrm{UV}}}{\mu}\right)
$$

where $r_{0 a}$ denotes bare parameters, $\Lambda_{\mathrm{UV}}$ is an ultraviolet cutoff, and $\mu$ is a finite energy scale. Via integration of the beta functions of the FI parameters, $\beta_{a}=\mu \frac{d r_{a}}{d \mu}$, the $\mu$ dependence is found to be

$$
r_{a}(\mu)=\sum_{i=1}^{N} \mathcal{Q}_{i a} \log \left(\frac{\mu}{\Lambda}\right),
$$

where $\Lambda$ is the renormalization group invariant dynamical scale. The second quantum effect is the anomaly of the bulk $U(1)$ axial $R$ symmetry, whereby axial $R$ rotations $\psi_{ \pm i} \rightarrow e^{\mp i \beta} \psi_{ \pm i}, \quad \sigma_{a} \rightarrow e^{2 i \beta} \sigma_{a}, \quad$ and $\lambda_{ \pm a} \rightarrow e^{\mp i \beta} \lambda_{ \pm a}$ no longer leave the action invariant, but result in a shift of the theta angles, i.e.,

$$
\theta_{a} \rightarrow \theta_{a}-2 \sum_{i=1}^{N} \mathcal{Q}_{i a} \beta
$$

The FI parameters are closely related to the boundary parameters $c_{i}$, via (5.18), and the latter undergo similar renormalization to that of (5.42) [10], i.e., the parameters $c_{i}$ run as

$$
c_{i}(\mu)=\log \left(\frac{\mu}{\Lambda}\right)
$$

Note that this quantum effect is nontrivial even when $\sum_{i=1}^{N} \mathcal{Q}_{i a}=0$, unlike the running of $r_{a}(\mu)$. In particular, (5.45) implies that the size of the equivariant A-brane in the toric manifold $X$ could depend on the energy scale $\mu$. However, for $\mathbb{C} P^{N-1}$, this is not the case, because the Dirichlet boundary condition is $\left|Z^{i}\right|^{2}=c_{i} / c_{N}$, and hence the equivariant A-brane stays the same size regardless of the energy scale. On the other hand, when $\sum_{i=1}^{N} \mathcal{Q}_{i a}>0$, the manifold $X$ becomes large at high energies due to (5.43), since $\hat{r}_{b}$ are the size moduli of $X$ [for $\mathbb{C} P^{N-1}$, this is obvious from (3.22)]. Finally, it is expected that in addition to the bulk axial $R$ anomaly, a boundary axial $R$ anomaly also occurs [20].

\section{Mirrors of equivariant A-branes}

Having described equivariant A-branes in toric manifolds, we shall now use mirror symmetry to find the Landau-Ginzburg mirrors of these branes, following the exposition in Sec. III, as well as the results of [10]. We shall obtain the mirrors of branes in toric manifolds that obey $c_{1}(X) \geq 0$, since mirror symmetry is a quantum duality (which holds after taking all perturbative and nonperturbative quantum effects into account), and we can only obtain quantum GNLSMs for Kähler targets with $c_{1}(X) \geq 0$ from GLSMs (cf. Sec. III).

The boundary action of the $U(1)^{N}$ GLSM that we wish to dualize is given by (5.23), with $\sum_{i}^{N} a_{i} \partial_{0} u_{i}$ replaced by $\sum_{i}^{N} a_{i} \partial_{0} \varphi_{i}$ via (5.24). ${ }^{35}$ The terms in the full $U(1)^{N}$ GLSM action relevant for the dualization are those that involve $\varphi_{i}$,

$$
\begin{aligned}
S_{\varphi}= & -\frac{1}{2 \pi} \sum_{i=1}^{N} \int_{\Sigma}\left|\phi_{i}\right|^{2}\left(\partial_{\mu} \varphi_{i}+\sum_{a}^{N} \mathcal{Q}_{i a} A_{\mu a}\right)\left(\partial^{\mu} \varphi_{i}+\sum_{a}^{N} \mathcal{Q}_{i a} A_{a}^{\mu}\right) d^{2} x \\
& +\frac{1}{2 \pi} \int_{\partial \Sigma}\left[\sum_{i=1}^{N}\left(-2 u_{i}^{\prime}\left|\phi_{i}\right|^{2}\left(\partial_{1} \varphi_{i}+\sum_{a}^{N} \mathcal{Q}_{i a} A_{1 a}\right)+a_{i} \partial_{0} \varphi_{i}\right)+\sum_{a}^{N} \theta_{a} A_{0 a}\right] d x^{0},
\end{aligned}
$$

where $-2\left|\phi_{i}\right|^{2}\left(\partial_{1} \varphi_{i}+\sum_{a}^{N} \mathcal{Q}_{i a} A_{1 a}\right)=i \bar{\phi}_{i} \stackrel{\leftrightarrow}{D}_{1} \phi_{i} \cdot{ }^{36}$ Here, the boundary theta term $\sum_{a}^{N} \frac{\theta_{a}}{2 \pi} \int_{\partial \Sigma} A_{0 a} d x^{0}$ has been included in order to maintain the gauge invariance; i.e., the gauge transformations $\varphi_{i} \rightarrow \varphi_{i}+\sum_{a}^{N} \mathcal{Q}_{i a} \alpha_{a}, A_{\mu a} \rightarrow A_{\mu a}-\partial_{\mu} \alpha_{a}$ leave the expression (5.46) invariant [as long as (5.21) holds]. All other terms, including those involving fermions, have been suppressed for simplicity.

Now, let us consider a system of $N$ one-form fields $\left(B_{i}\right)_{\mu}$, as well as $N+N$ periodic scalar fields consisting of $\vartheta_{i}$ and $\tilde{u}_{i}$ with the action

$$
S^{\prime}=\frac{1}{2 \pi} \sum_{i=1}^{N}\left[\int_{\Sigma}\left(-\left|\phi_{i}\right|^{2} B_{i \mu} B_{i}^{\mu} d^{2} x-B_{i} \wedge d \vartheta_{i}+\sum_{a}^{N} \mathcal{Q}_{i a} \vartheta_{i} F_{a}\right)+\int_{\partial \Sigma}\left(a_{i}-\vartheta_{i}\right) \partial_{0} \tilde{u}_{i} d x^{0}\right]
$$

where $F_{a}$ is the curvature of $A_{a}=A_{\mu a} d x^{\mu}, F_{a}=d A_{a}$. In addition, the boundary condition

$$
\left(B_{i}\right)_{1}=0
$$

\footnotetext{
${ }^{35}$ In the following analysis, we shall take $\left|\phi_{i}\right|^{2}$ to be nonzero, and $\varphi_{i}=\operatorname{Im} \log \phi_{i}$ is understood to be well defined, permitting us to set $U_{i}=\varphi_{i}+U_{i}^{\prime}$, whereby $U_{i}^{\prime}$ is a boundary superfield that is single valued.

${ }^{36}$ The subsequent dualization analysis follows from that given in [10], the only difference being that we have generalized the gauge group from $U(1)$ to $U(1)^{N}$.
} 
is imposed. Integrating out $\vartheta_{i}$ gives rise to the constraints

$$
\begin{aligned}
d B_{i} & =\sum_{a}^{N} \mathcal{Q}_{i a} F_{a} \text { on } \Sigma, \\
\left(B_{i}\right)_{0} & =\partial_{0} \tilde{u}_{i} \text { along } \partial \Sigma .
\end{aligned}
$$

The first of these constraints is solved by $B_{i}=d \varphi_{i}+$ $\sum_{a}^{N} \mathcal{Q}_{i a} A_{a}$, where $\varphi_{i}$ is a periodic scalar field of period $2 \pi{ }^{37}$ Then, the second constraint together with the boundary condition (5.48) implies the relations

$$
\begin{aligned}
& \partial_{0} \varphi_{i}+\sum_{a}^{N} \mathcal{Q}_{i a} A_{0 a}=\partial_{0} \tilde{u}_{i}, \\
& \partial_{1} \varphi_{i}+\sum_{a}^{N} \mathcal{Q}_{i a} A_{1 a}=0,
\end{aligned}
$$

on the boundaries. Inserting the first expression of (5.50) into (5.47) we obtain the action (5.46) without the $u_{i}^{\prime}$ dependent terms (using $\sum_{i=1}^{N} \mathcal{Q}_{i a} a_{i}=\theta_{a}$ ). The second condition in (5.50) is equivalent to the presence of the $u_{i}^{\prime}$-dependent terms, since integrating out $u_{i}^{\prime}$ imposes the second equation of (5.50).

Alternatively, integrating out the fields $B_{i}$ imposes

$$
\begin{aligned}
& \left(B_{i}\right)_{0}=\frac{-\partial_{1} \vartheta_{i}}{2\left|\phi_{i}\right|^{2}}, \\
& \left(B_{i}\right)_{1}=\frac{-\partial_{0} \vartheta_{i}}{2\left|\phi_{i}\right|^{2}},
\end{aligned}
$$

and we obtain

$$
S_{\vartheta}=\frac{1}{2 \pi} \sum_{i=1}^{N}\left[\int_{\Sigma}\left(-\frac{1}{4\left|\phi_{i}\right|^{2}} \partial_{\mu} \vartheta_{i} \partial^{\mu} \vartheta_{i} d^{2} x+\sum_{a}^{N} \mathcal{Q}_{i a} \vartheta_{i} F_{a}\right)+\int_{\partial \Sigma}\left(a_{i}-\vartheta_{i}\right) \partial_{0} \tilde{u}_{i} d x^{0}\right] .
$$

Following Hori [10], the bulk portion of the full mirror action is given by (3.2) [modulo boundary terms that arise from putting the scalar kinetic terms in (3.2) in their standard form], while the mirror boundary action takes the form ${ }^{38}$

$$
\begin{aligned}
S_{\partial \Sigma}= & \frac{1}{2 \pi} \sum_{i=1}^{N} \int_{\partial \Sigma} d x^{0} \operatorname{Re} \int d \theta\left(s_{i}-Y_{i}\right) \tilde{\Upsilon}_{i} \\
& +(\text { additional boundary terms required to cancel bulk SUSY variation }) \\
& +\sum_{a}^{N} \frac{1}{2 e_{a}^{2}} \int d \theta d \bar{\theta} \operatorname{Re}\left[\Xi_{a}\left(D_{+} \Sigma_{a}-\bar{D}_{-} \Sigma_{a}\right)\right]
\end{aligned}
$$

where the boundary term in (5.53) is contained in the first term.

Here, $\tilde{\Upsilon}_{i}$ is the "field strength" $\bar{D} \tilde{U}_{i}$ of the boundary superfield $\tilde{U}_{i}$, whose only difference from $U_{i}$ is that its lowest component is $\tilde{u}_{i}$. Integrating out $\tilde{\Upsilon}_{i}$, we find the boundary condition

$$
Y_{i}=s_{i}
$$

at $A$ boundary, which is

$$
\begin{aligned}
y_{i} & =s_{i}, \\
\bar{\chi}_{+i}-\chi_{-i} & =0
\end{aligned}
$$

\footnotetext{
${ }^{37}$ For details on why $\varphi_{i}$ ought to be periodic, see [20], page 250 .

${ }^{38}$ As explained in [10], unlike the bulk superpotential $\sum_{i=1}^{N} e^{-Y_{i}}$, which is generated by vortices, no boundary $\mathrm{F}$ terms can be generated by such effects.
}

in components. In fact, integrating out all the boundary auxiliary fields in (5.54) imposes the boundary conditions (5.55) and (5.13), which result in the entire boundary action vanishing.

As in Sec. III, taking the $\hat{e}_{b} \rightarrow \infty$ limit allows us to integrate $\hat{\Sigma}_{b}$ out of the action and imposes the constraint

$$
\sum_{j}^{N} \hat{Q}_{j b} Y_{j}-\hat{t}_{b}=0
$$

giving us the gauged Landau-Ginzburg theory with holomorphic twisted superpotential

$$
\tilde{W}=\sum_{c}^{k}\left(\sum_{j=1}^{N} \tilde{Q}_{j c} Y_{j}-\tilde{t}_{c}\right) \tilde{\Sigma}_{c}+\sum_{j=1}^{N} e^{-Y_{j}}
$$

We recall that the constraint (5.57) fixes the target space of the gauged Landau-Ginzburg theory to be the algebraic torus $\left(\mathbb{C}^{\times}\right)^{k}$. It is solved (cf. Sec. III) by 


$$
Y_{j}=\hat{s}_{j}+\sum_{c=1}^{k} v_{c j} \Theta_{c}
$$

where $\hat{s}_{j}$ is any solution of $\hat{Q}_{j}^{b} \hat{s}^{j}=\hat{t}^{b}$. Note that with $\Theta_{c}=\theta_{c}+\theta^{+} \bar{\chi}_{+c}^{\theta}+\bar{\theta}^{-} \chi_{-c}^{\theta}+\theta^{+} \bar{\theta}^{-} E_{c}^{\theta}$, the full mirror action, expanded in components, is

$$
\begin{aligned}
S= & \frac{1}{2 \pi} \int d^{2} x\left[\sum_{c}^{k} \sum_{d}^{k}\left(-g_{c d} \partial_{\mu} \theta_{c} \partial^{\mu} \bar{\theta}_{d}+\frac{i}{2} g_{c d} \bar{\chi}_{-c}^{\theta}\left(\stackrel{\leftrightarrow}{\partial}_{+}\right) \chi_{-d}^{\theta}+\frac{i}{2} g_{c d} \bar{\chi}_{+c}^{\theta}\left(\stackrel{\leftrightarrow}{\partial}_{-}\right) \chi_{+d}^{\theta}+g_{c d} E_{c}^{\theta} \bar{E}_{d}^{\theta}\right)\right. \\
& +\sum_{c}^{k} \frac{1}{2 \tilde{e}_{c}^{2}}\left(\left(\tilde{F}_{01 c}\right)^{2}-\partial_{\mu} \tilde{\sigma}_{c} \partial^{\mu} \overline{\tilde{\sigma}}_{c}+\left(\tilde{D}_{c}\right)^{2}+\frac{i}{2} \overline{\tilde{\lambda}}_{+c}\left(\stackrel{\leftrightarrow}{\partial}_{-}\right) \tilde{\lambda}_{+c}+\frac{i}{2} \overline{\tilde{\lambda}}_{-c}\left(\stackrel{\leftrightarrow}{\partial}_{+}\right) \tilde{\lambda}_{-c}\right) \\
& +\frac{1}{2}\left(\sum_{j}^{N} \sum_{c}^{k} \sum_{d}^{k} \tilde{Q}_{j c} v_{d}^{j}\left(\tilde{\sigma}_{c} E_{d}^{\theta}-i \overline{\tilde{\lambda}}_{+c} \chi_{-d}^{\theta}-i \tilde{\lambda}_{-c} \bar{\chi}_{+d}^{\theta}+\left(\tilde{D}_{c}-i \tilde{F}_{01 c}\right) \theta_{d}\right)\right. \\
& \left.\left.+\sum_{c}^{k}\left(\sum_{j}^{N} \tilde{Q}_{j c} \hat{s}^{j}-\tilde{t}_{c}\right)\left(\tilde{D}_{c}-i \tilde{F}_{01 c}\right)+\sum_{j}^{N} e^{-\sum_{c} v_{c}^{j} \theta_{c}-\hat{s}^{j}}\left(-\sum_{c}^{k} v_{c}^{j} \bar{\chi}_{+c}^{\theta} \sum_{d}^{k} v_{d}^{j} \chi_{-d}^{\theta}-\sum_{c}^{k} v_{c}^{j} E_{c}^{\theta}\right)+\text { c.c. }\right)\right],
\end{aligned}
$$

where $\left(\theta_{c}, \bar{\theta}_{d}\right)$ [the lowest components of $\left.\left(\Theta_{c}, \bar{\Theta}_{d}\right)\right]$ parametrize the mirror target space $\left(\mathbb{C}^{\times}\right)^{k}$, on which the flat Kähler metric is

$d s^{2}=\sum_{c}^{k} \sum_{d}^{k} \frac{1}{4} \frac{\sum_{j}^{N} v_{c}^{j} v_{d}^{j}}{\log \left(\Lambda_{\mathrm{UV}} / \mu\right)} d \theta_{c} d \bar{\theta}_{d}=\sum_{c}^{k} \sum_{d}^{k} g_{c d} d \theta_{c} d \bar{\theta}_{d}$

Now, the boundary condition (5.55) on $Y_{i}$ implies the boundary condition

$$
\sum_{c=1}^{k} v_{c j} \Theta_{c}=s_{j}-\hat{s}_{j}
$$

and this means that the $U(1)^{k}$-equivariant A-brane in $X=$ $\mathbb{C}^{N} / / U(1)^{N-k}$ is mapped to a B-brane that is a D0-brane in the mirror Landau-Ginzburg model located at $\theta_{c}$, where $\theta_{c}$ is a solution of $\sum_{c=1}^{k} v_{c j} \theta_{c}=s_{j}-\hat{s}_{j}{ }^{39}$ Let us investigate this D0-brane further, by studying how it is described in the $\tilde{W}$ plane. In particular, we would like to find the mirror of the $U(1)^{k}$-equivariant structure on the A-brane.

First, we note that the twisted superpotential (5.58) can be rewritten as

$$
\tilde{W}=\tilde{W}_{\text {equiv }}+\tilde{W}_{X},
$$

where the first and second terms of (5.58) correspond, respectively, to the first and second terms of (5.63). The image of the D0-brane in the $\tilde{W}_{X}$ plane is

\footnotetext{
${ }^{39}$ In the case where the two boundaries of the strip are mapped to different equivariant A-branes, labeled by $s_{j}^{\pi}$ and $s_{j}^{0}$, the positions of the mirror D0-branes are determined by $\sum_{c=1}^{k} v_{c j} \theta_{c}=s_{j}^{\pi}-\hat{s}_{j}$ and $\sum_{c=1}^{k} v_{c j} \theta_{c}=s_{j}^{0}-\hat{s}_{j}$, respectively.
}

$$
\tilde{W}_{X}=\sum_{i=1}^{N} e^{-s_{i}}
$$

which is the mirror condition found in [10] when studying the mirrors of A-branes without equivariant structure. However, turning to $\tilde{W}_{\text {equiv }}$, we find that the boundary condition (5.55) implies that the image of the D0-brane in the $\tilde{W}_{\text {equiv }}$ plane is $\tilde{W}_{\text {equiv }}=0$, and thus we require further analysis to identify the mirror of the $U(1)^{k}$-equivariant structure on the A-brane.

Now, for the D0-brane mirrors of ordinary A-branes, there is an additional requirement that is necessary to prevent spontaneous supersymmetry breaking; that is, the D0-brane should be at a critical point of the twisted superpotential $\tilde{W}_{X}(\theta)=\sum_{j=1}^{N} e^{-\hat{s}_{j}-\sum_{c=1}^{k} v_{c j} \theta_{c}}[10,20,27,28]$. This condition is necessary for the potential energy of the mirror Landau-Ginzburg model (with twisted superpotential $\tilde{W}_{X}$ ) to have a vanishing vacuum expectation value. We shall generalize this analysis to the gauged LandauGinzburg model with neutral matter (5.60), which we are presently concerned with. Here, the twisted superpotential terms can be expanded as

$$
\begin{aligned}
& \frac{1}{2 \pi} \int d^{2} x \frac{1}{2}\left(\int d^{2} \tilde{\theta} \tilde{W}(\Theta, \tilde{\Sigma})+\text { c.c. }\right) \\
& =\frac{1}{2 \pi} \int d^{2} x \frac{1}{2}\left(\sum_{c}^{k}\left(E_{c}^{\theta} \frac{\partial \tilde{W}}{\partial \theta_{c}}+\left(\tilde{D}_{c}-i \tilde{F}_{01 c}\right) \frac{\partial \tilde{W}}{\partial \tilde{\sigma}_{c}}\right)+\right.\text { c.c. } \\
& \quad+\sum_{c}^{k} \sum_{d}^{k}\left(\chi_{-c}^{\theta} \bar{\chi}_{+d}^{\theta} \frac{\partial^{2} \tilde{W}}{\partial \theta_{c} \partial \theta_{d}}+\tilde{\lambda}_{-c} \overline{\tilde{\lambda}}_{+d} \frac{\partial^{2} \tilde{W}}{\partial \tilde{\sigma}_{c} \partial \tilde{\sigma}_{d}}\right. \\
& \quad+i \bar{\chi}_{+c}^{\theta} \tilde{\lambda}_{-d} \frac{\left.\left.\partial^{2} \tilde{W}_{\partial \theta_{c} \partial \tilde{\sigma}_{d}}+i \chi_{-c}^{\theta} \overline{\tilde{\lambda}}_{+d} \frac{\partial^{2} \tilde{W}}{\partial \theta_{c} \partial \tilde{\sigma}_{d}}\right)+ \text { c.c. }\right)}{}
\end{aligned}
$$


where $\tilde{W}$ is given by (3.7). Taking into account the presence of the auxiliary field terms

$$
\frac{1}{2 \pi} \int d^{2} x\left(\sum_{c}^{k} \sum_{d}^{k} g_{c d} E_{c}^{\theta} \bar{E}_{d}^{\theta}+\sum_{c}^{k} \frac{1}{2 \tilde{e}_{c}^{2}} \tilde{D}_{c} \tilde{D}_{c}\right)
$$

in the action, upon integrating out the auxiliary fields $\tilde{D}_{c}$ and $E_{c}^{\theta}$, the potential energy becomes

$$
V=\frac{1}{2 \pi} \int d x^{1}\left(\frac{1}{4} g^{c d} \frac{\partial \tilde{W}}{\partial \theta_{c}} \frac{\partial \overline{\tilde{W}}}{\partial \bar{\theta}_{d}}+\frac{1}{2} \sum_{c}^{k} \tilde{e}_{c}^{2} \operatorname{Re}\left(\frac{\partial \tilde{W}}{\partial \tilde{\sigma}_{c}}\right) \operatorname{Re}\left(\frac{\partial \tilde{\tilde{W}}}{\partial \overline{\tilde{\sigma}}_{c}}\right)\right) .
$$

Now, in the nongauged case, $\frac{\partial \tilde{W}_{X}}{\partial \theta_{c}}$ is a constant at the boundaries, and therefore supersymmetry would be broken for any classical configuration unless the D0-brane is located at the critical point $\frac{\partial \tilde{W}_{X}}{\partial \theta_{c}}=0$. However, in (5.67),

$$
\frac{\partial \tilde{W}}{\partial \theta_{c}}=\sum_{j}^{N}\left\langle\tilde{\sigma}, \tilde{Q}_{j}\right\rangle v_{c}^{j}-\sum_{j}^{N} v_{c}^{j} e^{-\left\langle v^{j}, \theta\right\rangle-\hat{s}^{j}}
$$

is not a constant at the boundaries [since $\tilde{\sigma}_{c}$ obeys a Neumann boundary condition $\left(\partial_{1} \tilde{\sigma}_{c}=0\right)$, unlike $\theta_{c}$ ], and hence classical configurations where $\frac{\partial \tilde{W}}{\partial \theta_{c}}=0$ at the boundaries can be achieved without any additional constraint on the position of the D0-brane. Next, the second term in (5.67) implies that $\operatorname{Re}\left(\frac{\partial \tilde{W}}{\partial \tilde{\sigma}_{c}}\right)$ ought to vanish at each boundary in order to prevent spontaneous breaking of supersymmetry. Indeed,

$$
\operatorname{Re}\left(\frac{\partial \tilde{W}}{\partial \tilde{\sigma}_{c}}\right)=\operatorname{Re}\left(\sum_{j}^{N} \tilde{Q}_{j c} s^{j}-\tilde{t}_{c}\right)
$$

at the boundaries, which is identically zero because it is the real part of the condition $\sum_{j}^{N} \tilde{Q}_{j c} s^{j}-\tilde{t}_{c}=0$, which is implied by $\sum_{j}^{N} \mathcal{Q}_{j a} s^{j}-t_{a}=0$. The latter holds since it was necessary for the $A$-type supersymmetry and gauge symmetry of the $U(1)^{N-k} \times U(1)^{k}$ GLSM [see (5.22)]. Therefore, spontaneous supersymmetry breaking does not occur in the mirror theory, since zero-energy classical configurations can always be achieved at the boundaries. The condition $\sum_{j}^{N} \tilde{Q}_{j c} s^{j}-\tilde{t}_{c}=0$ is a new condition that did not appear in the nongauged case and, in fact, constrains the position of the D0-brane [defined by $s^{j}$ via (5.62)]. In conclusion, unlike the mirrors of ordinary A-branes, we have found the following:

The mirrors of $U(1)^{k}$-equivariant $A$-branes on $\mathbb{C}^{N} / / U(1)^{N-k}$ do not need to satisfy the critical point
condition $\frac{\partial \tilde{W}_{X}}{\partial \theta_{c}}=0$, but instead their position must be further constrained by $\sum_{j}^{N} \tilde{Q}_{j c} s^{j}-\tilde{t}_{c}=0$.

In this section, we have restricted ourselves to equivariant A-branes whose mirrors are D0-branes. However, there are A-branes whose mirrors are higher-dimensional branes holomorphically embedded in the mirror target space. In Hori's construction [10], these can be studied by promoting the parameter $s_{i}$ to a superfield $S_{i}$. It would be interesting to study equivariant structure on these branes.

\section{E. Non-Abelian equivariant A-branes}

We may use the insights obtained from analyzing the equivariant A-branes for Abelian groups to find the description of equivariant A-branes for general nonAbelian groups. We shall use the GNLSM notation of Sec. II in this subsection.

First, the terms in (2.17) [except the terms proportional to $\left(\phi^{*} \mu_{a}+r_{a}\right)$ and $\left.\theta_{a}\right]$ vanish using the boundary conditions

$$
\begin{aligned}
\lambda_{+a}-\bar{\lambda}_{-a} & =0, \\
\partial_{1} \sigma_{a} & =0, \\
A_{1 a} & =0, \\
\partial_{1} A_{0 a} & =0, \\
D_{a} & =0, \\
\partial_{1}\left(\lambda_{-a}+\bar{\lambda}_{+a}\right) & =0 .
\end{aligned}
$$

Note that these conditions are a direct generalization of the conditions given for the example of $\mathbb{C} P^{N-1}$, except that $F_{01 a}=0$ is replaced by the stricter conditions $A_{1 a}=0$ and $\partial_{1} A_{0 a}=0$. This is necessary since the supersymmetry transformations now contain non-Abelian terms, and this causes $A$-type supersymmetry invariance of the set of boundary conditions to not hold unless we use the stricter 
conditions. The boundary conditions, in fact, imply that gauge transformations have to be restricted such that the transformation parameter $\alpha^{a}$ has a vanishing derivative with respect to $x^{1}$ at the boundaries, in order for these boundary conditions to be gauge invariant.

Next, we turn to the boundary conditions for the matter fields. We first recall that for $\mathbb{C} P^{N-1}$, the equivariant A-brane corresponded to a Lagrangian torus $T^{N-1}$, which was invariant under the $U(1)^{N-1}$ isometry of $\mathbb{C} P^{N-1}$. Let us consider the $\mathcal{N}=1$ subalgebra of $A$-type supersymmetry, which corresponds to $\epsilon_{+}=i \tilde{\epsilon}, \bar{\epsilon}_{+}=-i \tilde{\epsilon}, \epsilon_{-}=-i \tilde{\epsilon}$, and $\bar{\epsilon}_{-}=i \tilde{\epsilon}$, where $\tilde{\epsilon}$ is a real parameter. In this case, after integrating out the auxiliary fields $F^{i}$ and $\bar{F}^{\overline{ }}$, we find that (2.16) and the $B$-field terms in (2.18) are

$$
\begin{aligned}
& -\frac{1}{2 \pi} \frac{i \tilde{\epsilon}}{2} \int_{\partial \Sigma} d x^{0}\left\{\left(g_{I J}\left(\psi_{-}^{J}-\psi_{+}^{J}\right)-B_{I J}\left(\psi_{-}^{J}+\psi_{+}^{J}\right)\right) \partial_{0}^{A} \phi^{I}\right. \\
& \quad+\left(g_{I J} \partial_{1}^{A} \phi^{I}+B_{J I} \partial_{0}^{A} \phi^{I}\right)\left(\psi_{-}^{J}+\psi_{+}^{J}\right) \\
& \left.\quad+g_{I J}\left(\psi_{+}^{I}-\psi_{-}^{I}\right) \operatorname{Re}\left(\sigma^{a}\right) \tilde{e}_{a}^{J}+\omega_{I J}\left(\psi_{+}^{I}+\psi_{-}^{I}\right) \operatorname{Im}\left(\sigma^{a}\right) \tilde{e}_{a}^{J}\right\},
\end{aligned}
$$

where $g_{I J} X^{I} Y^{J}=g_{i \bar{j}}\left(X^{i} Y^{\bar{\jmath}}+X^{\bar{j}} Y^{i}\right) \quad$ and $\quad \omega_{I J} X^{I} Y^{J}=$ $i g_{i \bar{\jmath}}\left(X^{i} Y^{\bar{\jmath}}-X^{\bar{\jmath}} Y^{i}\right)$, and where $(I, J, K, \ldots)$ are indices corresponding to real coordinates on $X$. In addition, if we insist on locality of the matter equations of motion (as in the $\mathbb{C} P^{N-1}$ case), we require that

$$
\begin{aligned}
\delta \phi^{I}\left(g_{I J} \partial_{1}^{A} \phi^{J}+B_{I J} \partial_{0}^{A} \phi^{J}\right) & =0, \\
g_{I J}\left(\psi_{-}^{I} \delta_{\nabla} \psi_{-}^{J}-\psi_{+}^{I} \delta_{\nabla} \psi_{+}^{J}\right) & =0
\end{aligned}
$$

at the boundaries, where $\delta_{\nabla} \psi^{J}=\delta \psi^{J}+\Gamma_{K L}^{J} \delta \phi^{K} \psi^{L}$. An equivariant A-brane shall wrap a submanifold (denoted as $\gamma$ ) of $X$, to which a boundary of the world sheet is mapped via $\left(\phi^{i}, \bar{\phi}^{\bar{i}}\right)$. Now, any allowed variation of $\phi$ (denoted $\delta \phi^{I}$ for the real coordinate $\phi^{I}$ ) along the boundary, and the derivative along the boundary, $\partial_{0} \phi^{I}$, ought to be tangent to $\gamma$. Hence, taking into account the fact that $A_{1 a}=0$ at the boundaries, we find that (5.71) vanishes while satisfying the first constraint of (5.72) if $\partial_{1} \phi^{J}$ is normal to $\gamma, \psi_{-}^{I}-\psi_{+}^{I}$ and $\psi_{-}^{I}+\psi_{+}^{I}$ are, respectively, normal and tangent to $\gamma, \tilde{e}_{a}^{I}$ is tangent to $\gamma$, the Kähler form vanishes against tangent vectors of $\gamma$, and the $B$ field vanishes against tangent vectors of $\gamma$. These last three conditions, respectively, imply that $\gamma$ is $G$ invariant, that it is an isotropic submanifold of $X$, and that the restriction of the two-form $B$ to $\gamma$ vanishes. In addition, we note that $\psi_{-}^{I}-\psi_{+}^{I}$ being normal to $\gamma$ and $\psi_{-}^{I}+\psi_{+}^{I}$ being tangent to $\gamma$ implies that

$$
\begin{array}{ll}
\psi_{-}^{I}-\psi_{+}^{I}=0, & I: \text { tangent to } \gamma, \\
\psi_{-}^{I}+\psi_{+}^{I}=0, & I: \text { normal to } \gamma
\end{array}
$$

(for a choice of coordinates that separates the normal and tangent directions), which satisfies the second constraint of (5.72).

Next, the $\mathcal{N}=(2,2)$ supersymmetry transformation of $\phi^{I}$ is

$\delta \phi^{I}=i\left(\epsilon_{+2} \psi_{-}^{I}-\epsilon_{+1} J_{K}^{I} \psi_{-}^{K}-\epsilon_{-2} \psi_{+}^{I}+\epsilon_{-1} J_{K}^{I} \psi_{+}^{K}\right)$,

where $\epsilon_{+}=\epsilon_{+1}+i \epsilon_{+2}$ and $\epsilon_{-}=\epsilon_{-1}+i \epsilon_{-2}$, and where $J$ is the almost complex structure of $X$ locally given by $J_{k}^{i}=i \delta_{k}^{i}$ and $J_{\bar{k}}^{\bar{l}}=-i \delta_{\bar{k}}^{\bar{i}}$. A-type supersymmetry corresponds to $\epsilon_{+1}=\epsilon_{-1}$ and $\epsilon_{+2}=-\epsilon_{-2}$, whereby

$$
\delta \phi^{I}=i\left(\epsilon_{+2}\left(\psi_{-}^{I}+\psi_{+}^{I}\right)-\epsilon_{+1} J_{K}^{I}\left(\psi_{-}^{K}-\psi_{+}^{K}\right)\right) .
$$

Hence, $\psi_{-}^{I}+\psi_{+}^{I}$ and $J_{K}^{I}\left(\psi_{-}^{K}-\psi_{+}^{K}\right)$ are tangent to $\gamma$. However, from the previous paragraph, we know that $\psi_{-}^{I}-\psi_{+}^{I}$ is normal to $\gamma$. In addition, $J^{M}{ }_{I} J_{K}^{I}=-\delta_{K}^{M}$. Hence, the application of the almost complex structure, $J$, converts normal vectors of $\gamma$ into tangent vectors of $\gamma$, and vice versa. Thus, $\gamma$ is a middle-dimensional Lagrangian submanifold of $X$. This Lagrangian submanifold also happens to be $G$ invariant, which we know from the previous paragraph.

Indeed, (2.16) and the $B$-field terms in (2.18) vanish under this boundary condition; integrating out the auxiliary fields $F^{i}$ and $\bar{F}^{\bar{\imath}}$, (2.16) and the $B$-field terms in (2.18) can be rewritten (for $\epsilon_{+}=\bar{\epsilon}_{-}=\epsilon$ ) as

$$
\begin{aligned}
\frac{1}{2 \pi} & \frac{1}{4} \int_{\partial \Sigma} d x^{0}\left\{\epsilon \left(-g\left(\partial_{0}^{A} \phi, \psi_{-}-\psi_{+}\right)-i \omega\left(\partial_{0}^{A} \phi, \psi_{-}+\psi_{+}\right)\right.\right. \\
& -g\left(\partial_{1}^{A} \phi, \psi_{-}+\psi_{+}\right)-i \omega\left(\partial_{1}^{A} \phi, \psi_{-}-\psi_{+}\right) \\
& -\operatorname{Re}\left(\sigma^{a}\right) g\left(\tilde{e}_{a}, \psi_{+}-\psi_{-}\right)-i \operatorname{Re}\left(\sigma^{a}\right) \omega\left(\tilde{e}_{a}, \psi_{+}+\psi_{-}\right) \\
& -i \operatorname{Im}\left(\sigma^{a}\right) g\left(\tilde{e}_{a}, \psi_{+}-\psi_{-}\right)+\operatorname{Im}\left(\sigma^{a}\right) \omega\left(\tilde{e}_{a}, \psi_{+}+\psi_{-}\right) \\
& \left.+2 B\left(\partial_{0}^{A} \phi, \psi_{-}+\psi_{+}\right)-2 i \omega^{-1}\left(g\left(\psi_{-}-\psi_{+}\right), B \partial_{0}^{A} \phi\right)\right) \\
& + \text { c.c. }\}
\end{aligned}
$$

[where $g(X, Y)=g_{I J} X^{I} Y^{J}, \omega(X, Y)=\omega_{I J} X^{I} Y^{J}, B(X, Y)=$ $B_{I J} X^{I} Y^{J}$, and $\left.\omega^{-1}(X, Y)=\omega^{I J} X_{I} Y_{J}\right]$, which vanishes using $A_{1 a}=0$ as well as the conditions that $\partial_{0} \phi^{I}, \psi_{-}^{I}+\psi_{+}^{I}$, and $\tilde{e}_{a}^{I}$ are tangent to $\gamma$ while $\partial_{1} \phi^{I}$ and $\psi_{-}^{I}-\psi_{+}^{I}$ are normal to $\gamma$, together with the condition that $\left.B\right|_{\gamma}=0 .{ }^{40}$

Next, we consider the terms proportional to $\left(\phi^{*} \mu_{a}+r_{a}\right)$ and $\theta_{a}$ in (2.17), as well as the term proportional to $\phi^{*} C_{a}$ in (2.18). Now, on a $G$-invariant Lagrangian submanifold, we have $\omega_{I J} \tilde{e}_{a}^{I} T^{J}=0$ for any tangent vector $T$. Using

\footnotetext{
${ }^{40}$ Recall that for a tangent vector, $T$, and normal vector, $N$, of a Lagrangian submanifold, $\gamma$, of the Kähler manifold $X$, we have $\omega(T, T)=\omega(N, N)=0$. Also, $\omega^{-1}(g N, B T)=0$ means that the restriction of $B$ to $(T \gamma)^{\circ} \times T \gamma$ vanishes, where $(T \gamma)^{\circ}$ is the subspace of $T X$ orthogonal to $T \gamma$ with respect to $\omega$. When $\gamma$ is a Lagrangian submanifold, then $(T \gamma)^{\circ}=T \gamma$, and $B$ vanishes when restricted to $\gamma$.
} 
$d \mu_{a}=l_{\tilde{e}_{a}} \omega$, this implies $\partial_{J} \mu_{a} T^{J}=0$; i.e., $\mu$ ought to be a constant along $\gamma$. Moreover, gauge invariance of the pullback of this condition to $\partial \Sigma$ requires that the constant be an element of $[\mathfrak{g}, \mathfrak{g}]^{0}$, via the identity $\alpha^{b} \mathcal{L}_{\tilde{e}_{b}} \mu_{a}=[\alpha, \mu]_{a}$ [3]. Choosing the constant to be

$$
\mu_{a}=-r_{a}
$$

we find that the terms proportional to $\left(\phi^{*} \mu_{a}+r_{a}\right)$ in (2.17) vanish. Analogously, the fact that $B_{I J} \tilde{e}_{a}^{I} T^{J}=0$ along $\gamma$ implies that $C$ ought to be a constant element of $[\mathfrak{g}, \mathfrak{g}]^{0}$ along $\gamma$. Choosing the constant to be

$$
C_{a}=\theta_{a},
$$

we find that the remaining term in (2.17) and the remaining term in (2.18) cancel. Note that the boundary conditions (5.70) together with the constraint (5.78) preserve the locality of the equations of motion for vector multiplet components.

Finally, we consider a boundary action. We note that the boundary action (5.41) for $\mathbb{C} P^{N-1}$ is an example of the GNLSM generalization of the NLSM boundary Wilson line (5.4). Hence, for general GNLSMs the boundary action ought to be

$$
\begin{aligned}
S_{\partial \Sigma} & =\int_{\partial \Sigma} d x^{0} A_{M}^{X} \partial_{0}^{A} \phi^{M} \\
& =\int_{\partial \Sigma} d x^{0}\left(A_{M}^{X} \partial_{0} \phi^{M}-\tilde{R}_{a} A_{0}^{a}\right)
\end{aligned}
$$

where $A^{X}$ corresponds to a $G$-invariant $\left(\mathcal{L}_{\tilde{e}} A^{X}=0\right)$ connection of a flat $\left(F_{M N}^{X}=0\right) U(1)$ bundle on each A-brane, and where $\tilde{R}_{a}=-l_{\tilde{e}_{a}} A^{X}$ (we shall use $(M, N, \ldots)$ as coordinate indices on the A-branes). ${ }^{41}$ Gauge invariance of this boundary action follows from the equivariant Bianchi identity

$$
d \tilde{R}=l_{\tilde{e}} F^{X},
$$

and this implies that each A-brane supports a flat, G-equivariant $U(1)$ bundle, for which $\tilde{R}_{a}$ is the moment. ${ }^{42}$ Its supersymmetry variation is

$$
\delta S_{\partial \Sigma}=-\int_{\partial \Sigma} d x^{0} \tilde{R}_{a}\left(\frac{i}{2}\left(\epsilon\left(\bar{\lambda}_{+}^{a}+\lambda_{-}^{a}\right)+\bar{\epsilon}\left(\lambda_{+}^{a}+\bar{\lambda}_{-}^{a}\right)\right)\right),
$$

where we have used (5.80). Just as in the $\mathbb{C} P^{N-1}$ case, we require that this cancels the $C$ term in (2.18) and the $\theta_{a}$ term in (2.17); i.e., we require that

$$
2 \pi \tilde{R}_{a}=-\theta_{a}+C_{a}
$$

on $\gamma$, the pullback of which is a gauge invariant condition on $\partial \Sigma$. This modification of (5.78) [together with the boundary conditions (5.70)] also preserves the locality of the equations of motion for vector multiplet components, just as in the example of $\mathbb{C} P^{N-1}$. In conclusion, we find that in general,

Equivariant $A$-branes are $G$-invariant Lagrangian submanifolds of $X$, which support $G$-equivariant
flat $U(1)$ bundles, and on which the restriction of the $B$-field vanishes.

This implies that they are objects in the $G$-equivariant Fukaya category of $X$, by generalizing the definition of the equivariant Fukaya category for finite groups ([26], page 68) to any compact Lie group $G$. Hence, we have further verified the conjecture of Kapustin et al. ([11], page 58) for non-Abelian $G$. Fully proving their conjecture would require constructing the other objects in the category, which correspond to Lagrangian submanifolds that support equivariant flat unitary vector bundles, and these should correspond to the insertion of certain $G$-invariant Wilson lines in the path integral.

\footnotetext{
${ }^{41}$ Note that the inclusion of this boundary action does not modify the constraints (5.72), since it vanishes under arbitrary variations of $\phi^{M}$ because $F_{M N}=0$.

${ }^{42}$ Note that gauge invariance of the boundary action requires the use of the identity $\alpha^{b} \mathcal{L}_{\tilde{e}_{b}} \tilde{R}_{a}=[\alpha, \tilde{R}]_{a}$.
}

\section{OPEN HAMILTONIAN GROMOV-WITTEN INVARIANTS}

In this section, we shall use equivariant A-branes to define open Hamiltonian Gromov-Witten invariants. We shall first study the non-Abelian invariants via the open topological gauged $A$ model, using the boundary conditions and boundary term we have found in Sec. V. E. In the final two subsections, we shall focus on investigating the Abelian open Hamiltonian Gromov-Witten invariants via mirror symmetry.

\section{A. Open topological gauged $\boldsymbol{A}$ model}

The closed topological gauged $A$ model was introduced by Baptista [6], and in the following we shall generalize it to the case with boundaries, i.e., the open topological gauged $A$ model. This involves analytically continuing the Minkowski strip to the Euclidean one, subsequently 
twisting the fields in the action (2.2) as well as its supercharges using their vector $\mathrm{R}$ charges, and imposing the appropriate boundary conditions (found in Sec. V. E) that are supersymmetric with respect to the scalar supercharge $Q_{A}=Q_{-}+\bar{Q}_{+}$. We also include the gauge invariant boundary term (5.79).

The twisted fields are redefined as follows:

$$
\begin{aligned}
\chi^{k} & =\sqrt{2} \psi_{-}^{k}, \quad \psi_{z}^{a}=\left(-i \lambda_{-}^{a}\right) / \sqrt{2}, \\
\bar{\chi}^{\bar{k}} & =\sqrt{2} \bar{\psi}_{+}^{\bar{k}}, \quad \psi_{\bar{z}}^{a}=\left(i \bar{\lambda}_{+}^{a}\right) / \sqrt{2}, \\
\varphi^{a} & =-i 2 \sigma^{a}, \quad \rho_{\bar{z}}^{k}=\sqrt{2} \psi_{+}^{k}, \\
\xi^{a} & =\bar{\sigma}^{a} / 4, \quad \bar{\rho}_{z}^{\bar{k}}=\sqrt{2} \bar{\psi}_{-}^{\bar{k}}, \\
\eta^{a} & =-i\left(\bar{\lambda}_{-}^{a}+\lambda_{+}^{a}\right) /(2 \sqrt{2}), \quad \kappa^{a}=i\left(\bar{\lambda}_{-}^{a}-\lambda_{+}^{a}\right) / \sqrt{2}, \\
\mathcal{H}_{\bar{z}}^{k} & =4 i d_{\bar{z}}^{A} \phi^{k}+2\left(F^{k}-\Gamma_{i j}^{k} \psi_{+}^{i} \psi_{-}^{j}\right), \quad \mathcal{C}^{a}=2\left(F_{A}\right)_{12}^{a}+2 D^{a},
\end{aligned}
$$

and further details on the twisting are given in $[6,17]$. The action of the open gauged $A$ model is then ${ }^{43}$

$$
\begin{aligned}
S_{A}= & \frac{1}{2 \pi} \int_{\Sigma}\left\{\frac{1}{2 e^{2}}\left|F_{A}\right|^{2}+\left|d^{A} \phi\right|^{2}+\frac{1}{2} e^{2}|\mu \circ \phi+r|^{2}+\frac{i}{e^{2}}\left(\nabla^{A} \varphi, \nabla^{A} \xi\right)+\frac{1}{2 e^{2}}|[\varphi, \xi]|^{2}\right. \\
& +\frac{1}{2 e^{2}}[\varphi, \eta]_{a} \eta^{a}-\frac{1}{8 e^{2}}[\varphi, \kappa]_{a} \kappa^{a}-\frac{1}{2 e^{2}}\left|\frac{1}{2} \mathcal{C}-* F_{A}-e^{2}(\mu \circ \phi+r)\right|^{2} \\
& -\frac{1}{4}\left|\mathcal{H}-4 i \bar{\partial}^{A} \phi\right|^{2}+i g_{j \bar{k}}\left(\varphi^{a} \xi^{b}+\varphi^{b} \xi^{a}\right) \tilde{e}_{a}^{j} \bar{e}_{b}^{\bar{k}}+2 i g_{j \bar{k}}\left(\nabla_{l} \tilde{e}_{a}^{j}\right) \xi a \chi^{l} \bar{\chi}^{\bar{k}} \\
& \left.+i g_{j \bar{k}}\left(\eta^{a}+\frac{1}{2} \kappa^{a}\right) \overline{\tilde{e}}_{a}^{\bar{k}} \chi^{j}+i g_{j \bar{k}}\left(\eta^{a}-\frac{1}{2} \kappa^{a}\right) \tilde{e}_{a}^{j} \bar{\chi}^{\bar{k}}\right\} \operatorname{vol}_{\Sigma} \\
& +\frac{1}{2 \pi} \int_{\Sigma}\left\{\frac{i}{e^{2}} \eta_{a} \nabla^{A} * \psi^{a}-\frac{1}{2 e^{2}} \kappa_{a} \nabla^{A} \psi^{a}-\frac{i}{8} R_{i \bar{j} k \bar{m}}\left(\rho^{i} \wedge \bar{\rho}^{\bar{j}}\right) \chi^{k} \bar{\chi}^{\bar{m}}\right. \\
& \left.+\frac{i}{e^{2}} \xi_{a}[\psi, * \psi]\right]^{a}+\frac{1}{2} g_{j \bar{k}} \rho^{j} \wedge\left(\phi^{*} \nabla^{A}\right) \bar{\chi}^{\bar{k}}+\frac{1}{2} g_{j \bar{k}} \bar{\rho}^{\bar{k}} \wedge\left(\phi^{*} \nabla^{A}\right) \chi^{j} \\
& \left.+\frac{i}{8} g_{j \bar{k}} \varphi^{a}\left(\nabla_{l} \tilde{e}^{j}\right) \rho^{l} \wedge \bar{\rho}^{\bar{k}}+\frac{1}{2} g_{j \bar{k}} \tilde{e}_{a}^{j} \psi^{a} \wedge \bar{\rho}^{\bar{k}}+\frac{1}{2} g_{j \bar{k}} \overline{\tilde{e}}_{a}^{\bar{k}} \psi^{a} \wedge \rho^{j}\right\} \\
& +\frac{1}{2 \pi} i\left(\int_{\Sigma} \phi^{*} B-\int_{\partial \Sigma} \phi^{*} C_{a} A^{a}\right)+\frac{1}{2 \pi} i \int_{\Sigma}\left(\theta, F_{A}\right)-i \int_{\partial \Sigma} A_{M}^{X} d^{A} \phi^{M}
\end{aligned}
$$

where $F_{A}$ is the curvature two-form of the connection $A=A_{\mu} d x^{\mu}$, and the measure on the world sheet is $\operatorname{vol}_{\Sigma}=d x \wedge d \tau=\frac{i}{2} d z \wedge d \bar{z}^{44,45}$ The fields $\mathcal{C}$ and $\mathcal{H}$ are auxiliary fields, which can be integrated out of the action using their equations of motion

$$
\begin{gathered}
\mathcal{C}^{a}=2 * F_{A}^{a}+2 e^{2}\left(\mu^{a} \circ \phi+r^{a}\right), \\
\mathcal{H}_{\bar{z}}^{k}=4 i d_{\bar{z}}^{A} \phi^{k} .
\end{gathered}
$$

The supersymmetry transformations generated by the scalar supercharge $Q_{A}=Q_{-}+\bar{Q}_{+}$on the new fields follow from the supersymmetry transformations (2.13) and (2.14), with $\epsilon_{+}=\bar{\epsilon}_{-}=\sqrt{2}$ and $\epsilon_{-}=\bar{\epsilon}_{+}=0$, which gives

\footnotetext{
${ }^{43}$ Here, we follow the notation of [6].

${ }^{44}$ Recall that when $G=G_{1} \times G_{2} \times G_{3} \ldots$, each factor $G_{i}$ has its own coupling constant, $e_{i}$, e.g., for $G=U(1)^{k}$, each $U(1)$ factor had its own coupling constant $\tilde{e}_{c}$.

${ }^{45}$ Note that we have performed integration by parts to undo the symmetrized form of the fermionic kinetic terms present in $(2.2)$, which is no longer necessary since a Euclidean action is not real. The resulting boundary terms vanish using boundary conditions found in Sec. V.E.
} 


$$
\begin{aligned}
Q_{A} \phi^{k} & =\chi^{k}, \quad Q_{A} A=\psi, \\
Q_{A} \chi^{k} & =\varphi^{a} \tilde{e}_{a}^{k}, \quad Q_{A} \psi=-\nabla^{A} \varphi, \\
Q_{A} \xi & =\eta \quad Q_{A} \kappa=\mathcal{C} \\
Q_{A} \eta & =[\varphi, \xi], \quad Q_{A} \mathcal{C}=[\varphi, \kappa], \\
Q_{A} \rho^{k} & =\mathcal{H}^{k}-\Gamma_{i j}^{k} \chi^{i} \rho^{j}, \quad Q_{A} \varphi=0, \\
Q_{A} \mathcal{H}^{k} & =-R_{i \bar{\jmath} l \bar{m}} g^{k \bar{\jmath}} \chi^{l} \bar{\chi}^{\bar{m}} \rho^{i}-\Gamma_{j l}^{k} \mathcal{H}^{j} \chi^{l}+\varphi^{a}\left(\nabla_{j} \tilde{e}_{a}^{k}\right) \rho^{j} .
\end{aligned}
$$

The action (6.2) is, in fact, $Q_{A}$ exact up to topological terms,

$$
S_{A}=Q_{A} \Psi+\frac{1}{2 \pi} \int_{\Sigma} \phi^{*}\left(\left[\eta_{\omega}\right]+i\left[\eta_{B}\right]\right)+\frac{1}{2 \pi} i \int_{\Sigma}\left(\theta, F_{A}\right)-i \int_{\partial \Sigma} A_{M}^{X} d^{A} \phi^{M}
$$

with gauge fermion

$$
\begin{aligned}
\Psi= & \frac{1}{2 \pi} \int_{\Sigma}\left\{\frac{1}{2 e^{2}} \kappa_{a}\left(* F_{A}+e^{2}(\mu \circ \phi+r)\right)^{a}-\frac{1}{8 e^{2}} \kappa_{a} \mathcal{C}^{a}+\frac{1}{2 e^{2}} \eta_{a}[\varphi, \xi]^{a}+i g_{j \bar{k}} \xi^{a}\left(\tilde{e}_{a}^{j} \bar{\chi}^{\bar{k}}+\overline{\tilde{e}}_{a}^{\bar{k}} \chi^{j}\right)\right\} \operatorname{vol}_{\Sigma} \\
& +\frac{1}{2 \pi} \int_{\Sigma}\left\{\frac{i}{e^{2}} \xi_{a}\left(\nabla^{A} * \psi^{a}\right)-\frac{i}{16} g_{j \bar{k}} \bar{\rho}^{\bar{k}} \wedge\left(\mathcal{H}-8 i \bar{\partial}^{A} \phi\right)^{j}+\frac{i}{16} g_{j \bar{k}} \rho^{j} \wedge\left(\mathcal{H}-8 \bar{i} \bar{\partial}^{A} \phi\right)^{k}\right\},
\end{aligned}
$$

where we have performed integration by parts in (6.2) such that $\nabla_{\mu}^{A} \varphi_{a} \nabla^{A \mu} \xi^{a}$ becomes $-\xi_{a} \nabla_{\mu}^{A} \nabla^{A \mu} \varphi^{a}$. The resulting boundary term vanishes using the boundary conditions $\partial_{1} \varphi_{a}=0$ and $A_{1 a}=0$ found in Sec. V. E.

Let us elucidate the first topological term of (6.6). Here, $\left[\eta_{\omega}\right]$ and $\left[\eta_{B}\right]$ are the cohomology classes in $H^{2}(E)$ represented by the two-forms

$$
\begin{aligned}
& \eta_{\omega}(A)=\omega-d\left(\left(\mu_{a}+r_{a}\right) A^{a}\right) \quad \in \Omega^{2}(P \times X), \\
& \eta_{B}(A)=B-d\left(C_{a} A^{a}\right) \quad \in \Omega^{2}(P \times X),
\end{aligned}
$$

both of which descend to $E=P \times_{G} X$. In particular, this term is topological since $\int_{\Sigma} \phi^{*}\left[\eta_{\omega}\right]$ and $\int_{\Sigma} \phi^{*}\left[\eta_{B}\right]$ do not change under deformations of the map $\phi$, since the pullback map is always homotopy invariant. In addition, the cohomology classes $\left[\eta_{\omega}\right]$ and $\left[\eta_{B}\right]$ are the pullbacks of the equivariant cohomology classes in $H_{G}^{2}(X)$ represented by $\omega-(\mu+r)$ and $B-C{ }^{46}$

The open gauged $A$ model is topological as a quantum theory, ${ }^{47}$ and in order to consistently quantize such a gauge theory, one ought to perform Becchi-Rouet-Stora-Tyutin (BRST) gauge fixing, which involves the inclusion of Faddeev-Popov ghost fields in the action. This can be done straightforwardly, and we shall not write down the gauge-fixing action, $S_{\mathrm{BRST}}$, explicitly. However, the open

\footnotetext{
${ }^{46}$ This follows because $H_{G}^{2}(X)=H^{2}\left(E G \times_{G} X\right)$, and since $P \times_{G} X \rightarrow \Sigma$ is the pullback bundle of $E G \times_{G} X \rightarrow B G$ via a map $\bar{U}: \sum \rightarrow B G$, where $E G \rightarrow B G$ is the universal bundle [29].

${ }^{47}$ In particular, the correlation functions of the theory are invariant under diffeomorphisms of the world sheet, e.g., transforming it from a strip to a disk.
}

gauged $A$ model is anomalous, and in Sec. VI. D, we shall compute this anomaly by canonically quantizing the gauged Landau-Ginzburg mirror of the Abelian open gauged $A$ model. Notably, in the process we shall describe $S_{\mathrm{BRST}}$ for Abelian gauge groups in detail.

\section{B. Observables and open Hamiltonian Gromov-Witten invariants}

A canonical set of bulk observables of the closed gauged $A$ model were described in [6], with the path integrals over these observables eventually argued to be equal to the Hamiltonian Gromov-Witten invariants. In this section, we shall recall the description of these bulk observables, as well as introduce boundary observables that are defined with respect to the topology of the equivariant A-branes.

In the ordinary open $A$ model, one can construct bulk observables from the de Rham cohomology classes of the target $X$, as well as construct boundary observables from the de Rham cohomology classes of the A-branes, which wrap the subspaces of $X$ (i.e., Lagrangian submanifolds) to which boundaries of the world sheet are mapped. For the open gauged $A$ model, one uses the $G$-equivariant cohomology classes of $X$ to define bulk operators, as well as the $G$-equivariant cohomology classes of the equivariant A-branes to define boundary operators.

The $G$-equivariant cohomology classes of a manifold, $M$, are defined using the $G$-equivariant complex $\Omega_{G}^{*}(M)$, which is the set of $G$-invariant elements in the tensor product $S^{*}\left(\mathfrak{g}^{*}\right) \otimes \Omega^{*}(M)$, with $S^{*}\left(\mathfrak{g}^{*}\right)$ being the symmetric algebra of the dual of $\mathfrak{g}$.

For $M=X$, an equivariant form, $\alpha$, can be written on a local patch of $X$ as 


$$
\alpha=\alpha_{a_{1} \ldots a_{r} k_{1} \ldots k_{p} \bar{l}_{1} \ldots \bar{l}_{q}}(w) \xi^{a_{1}} \ldots \xi^{a_{r}} d w^{k_{1}} \wedge \ldots \wedge d w^{k_{p}} \wedge d \bar{w}^{\overline{l_{1}}} \wedge \ldots \wedge d \bar{w}^{\overline{l_{q}}}
$$

where $\left(w^{k_{i}}, \bar{w}^{\bar{l}_{i}}\right)$ are the coordinates on the patch. The coefficients $\alpha_{a_{1} \ldots a_{r} k_{1} \ldots k_{p} \bar{l}_{1} \ldots \bar{l}_{q}}$ are symmetric with respect to the indices $a_{i}$ and antisymmetric with respect to the indices $k_{i}$ and $\bar{l}_{i}$. Such a local form can be associated with a bulk operator $\mathcal{O}_{\alpha}$ in the open gauged $A$ model,

$$
\mathcal{O}_{\alpha}=\left(\alpha_{a_{1} \ldots a_{r} k_{1} \ldots k_{p} \bar{l}_{1} \ldots \bar{l}_{q}} \circ \phi\right)\left[\prod_{j=1}^{r}\left(\varphi+\psi-F_{A}\right)^{a_{j}}\right]\left[\prod_{i=1}^{p}\left(\chi^{k_{i}}-d^{A} \phi^{k_{i}}\right)\right]\left[\prod_{i=1}^{q}\left(\bar{\chi}^{\bar{l}_{i}}-d^{A} \bar{\phi}^{\bar{l}_{i}}\right)\right] .
$$

This correspondence holds globally on $X$. Moreover, we have

$$
\left(d_{\Sigma}+Q_{A}\right) \mathcal{O}_{\alpha}=\mathcal{O}_{d_{G} \alpha}
$$

where $d_{\Sigma}$ is the exterior derivative on the open world sheet, $\Sigma$, while $d_{G}=1 \otimes d+e^{a} \otimes l_{\tilde{e}_{a}}$ is the Cartan operator defined on $\Omega_{G}^{\bullet}(X) . \mathcal{O}_{\alpha}$ can be decomposed with respect to the form degree on the world sheet,

$$
\mathcal{O}_{\alpha}=\mathcal{O}_{\alpha}^{(0)}+\mathcal{O}_{\alpha}^{(1)}+\mathcal{O}_{\alpha}^{(2)}
$$

where, in particular,

$$
\mathcal{O}_{\alpha}^{(0)}=\left(\alpha_{a_{1} \ldots a_{r} k_{1} \ldots k_{p} \bar{l}_{1} \ldots \bar{l}_{q}} \circ \phi\right)\left(\prod_{j=1}^{r} \varphi^{a_{j}}\right)\left(\prod_{i=1}^{p} \chi^{k_{i}}\right)\left(\prod_{i=1}^{q} \bar{\chi}^{\bar{l}_{i}}\right)
$$

is a local operator.

If we assume that $d_{G} \alpha=0$, then (6.10) splits into the descent equations

$$
\begin{gathered}
d_{\Sigma} \mathcal{O}_{\alpha}^{(2)}=0, \\
d_{\Sigma} \mathcal{O}_{\alpha}^{(1)}=-Q_{A} \mathcal{O}_{\alpha}^{(2)}, \\
d_{\Sigma} \mathcal{O}_{\alpha}^{(0)}=-Q_{A} \mathcal{O}_{\alpha}^{(1)}, \\
Q_{A} \mathcal{O}_{\alpha}^{(0)}=0 .
\end{gathered}
$$

For a closed world sheet, $\Sigma$, if $\beta$ is a $j$-dimensional homology cycle in $\Sigma$, one could define the $Q_{A}$-invariant operators

$$
W(\alpha, \beta):=\int_{\beta} \mathcal{O}_{\alpha}^{(j)}
$$

However, there are no 2-cycles on an open world sheet. Hence, $\mathcal{O}_{\alpha}^{(2)}$ ought to be integrated over the entire open world sheet, and it is necessary for $Q_{A}$ invariance of $\mathcal{O}_{\alpha}^{(2)}$ that $\mathcal{O}_{\alpha}^{(1)}=0$ at the boundaries.

For $M=L$, where $L$ is an equivariant A-brane (to which a boundary component $\partial \Sigma_{L}$ is mapped), an equivariant form, $\zeta$, can be written on a local patch of $L$ as

$$
\zeta=\zeta_{a_{1} \ldots a_{r} m_{1} \ldots m_{s}}(u) \xi^{a_{1}} \ldots \xi^{a_{r}} d u^{m_{1}} \wedge \ldots \wedge d u^{m_{s}},
$$

where $u^{m_{i}}$ are the coordinates on the patch. The coefficients $\zeta_{a_{1} \ldots a_{r} m_{1} \ldots m_{s}}$ are symmetric with respect to the indices $a_{i}$ and antisymmetric with respect to the indices $m_{i}$. Such a local form can be associated with a boundary operator $\left.\mathcal{O}_{\zeta}\right|_{\partial \Sigma_{L}}$ in the open gauged $A$ model,

$$
\begin{aligned}
\left.\mathcal{O}_{\zeta}\right|_{\partial \Sigma_{L}}= & \left(\zeta_{a_{1} \ldots a_{r} m_{1} \ldots m_{s}} \circ \gamma\right)\left[\prod_{j=1}^{r}\left(\varphi+\left.\psi\right|_{\partial \Sigma_{L}}\right)^{a_{j}}\right] \\
& \times\left[\prod_{i=1}^{s}\left(\left(Q_{A} \gamma\right)^{m_{i}}-d^{A} \gamma^{m_{i}}\right)\right],
\end{aligned}
$$

where $\gamma$ is a section $\gamma: \partial \Sigma_{L} \rightarrow E_{L}$ of the associated bundle $E_{L}=P_{\partial \Sigma_{L}} \times_{G} L$ (which looks like a map $\gamma: \partial \Sigma_{L} \rightarrow L$ locally on $\partial \Sigma_{L}$ ), where $P_{\partial \Sigma_{L}}$ is the principal $G$ bundle over $\partial \Sigma_{L}$, and where $\left.\psi\right|_{\partial \Sigma_{L}}$ is the restriction of $\psi$ to the boundary in question. In particular, we have

$$
\left(d_{\partial \Sigma_{L}}+Q_{A}\right) \mathcal{O}_{\zeta}=\mathcal{O}_{d_{G} \zeta}
$$

where $d_{\partial \Sigma_{L}}$ is the exterior derivative on the world sheet boundary $\partial \Sigma_{L}$, while $d_{G}$ is the Cartan operator defined on $\Omega_{G}^{\bullet}(L)$. Just as bulk operators, $\mathcal{O}_{\zeta}$ can be decomposed with respect to the form degree on the world sheet boundary, 


$$
\mathcal{O}_{\zeta}=\mathcal{O}_{\zeta}^{(0)}+\mathcal{O}_{\zeta}^{(1)}
$$

If it is assumed that $d_{G} \zeta=0$, then (6.18) splits into the descent equations

$$
\begin{gathered}
d_{\partial \Sigma_{L}} \mathcal{O}_{\zeta}^{(1)}=0, \\
d_{\partial \Sigma_{L}} \mathcal{O}_{\zeta}^{(0)}=-Q_{A} \mathcal{O}_{\zeta}^{(1)}, \\
Q_{A} \mathcal{O}_{\zeta}^{(0)}=0,
\end{gathered}
$$

where, for example,
$\mathcal{O}_{\zeta}^{(0)}=\left(\zeta_{a_{1} \ldots a_{r} m_{1} \ldots m_{s}} \circ \gamma\right)\left(\prod_{j=1}^{r} \varphi^{a_{j}}\right)\left(\prod_{i=1}^{s}\left(Q_{A} \gamma\right)^{m_{i}}\right)$.

Thus, if $\nu$ is a $j$-dimensional homology cycle in $\partial \Sigma_{L},{ }^{48}$ one can then define the $Q_{A}$-invariant operators

$$
W_{\partial \Sigma_{L}}(\zeta, \nu):=\int_{\nu} \mathcal{O}_{\zeta}^{(j)}
$$

The most general correlation function based on the above bulk and boundary operators can then be written down (for $\Sigma=I \times \mathbb{R})$ as the following path integral:

$$
\int \mathcal{D}(A, \phi, \varphi, \xi, \rho, \eta, \kappa, \psi, \chi, b, c) e^{-\left(S_{A}+S_{\mathrm{BRST}}\right)} \prod_{i} W\left(\alpha_{i}, \gamma_{i}\right) \prod_{j} W_{\partial \Sigma_{0}}\left(\zeta_{j}, \nu_{j}\right) \prod_{k} W_{\partial \Sigma_{\pi}}\left(\zeta_{k}^{\prime}, \nu_{k}^{\prime}\right),
$$

where $b$ and $c$ are ghost fields that appear in $S_{\text {BRST }}$.

Now, any supersymmetric path integral localizes to the bosonic field configurations that are fixed points of the supersymmetry [20]. For the open gauged $A$ model, these field configurations can be read from the $Q_{A}$ variations of the fermionic fields in (6.5), after integrating out the auxiliary fields. They correspond to the solutions of

$$
\begin{aligned}
\bar{\partial}^{A} \phi & =0, \\
* F_{A}+e^{2}(\mu \circ \phi+r) & =0, \\
\nabla^{A} \varphi=\varphi^{a}\left(\tilde{e}_{a} \circ \phi\right) & =0 .
\end{aligned}
$$

The first two equations are known as the symplectic vortex equations on an infinite strip, and were introduced by Cieliebak et al. in [3], and they are a generalization of the typical Nielsen-Olsen vortex equations on a strip. In what follows, we shall refer to them as the open symplectic vortex equations. The last two equations are nontrivial, but in the most interesting cases that we will consider have the trivial solution $\varphi=0$ [6], and therefore we can ignore them in these cases. For the first two equations of (6.25), the boundary condition used by Cieliebak et al. on the strip was that each boundary component of the strip was mapped to a $G$-invariant Lagrangian submanifold of $X$, and this is precisely the boundary condition we found in Sec. V. E. In addition, for the second equation, we have found the boundary conditions $A_{1 a}=0, \partial_{1} A_{0 a}=0$, and $\mu_{a}=-r_{a}$. For the example of $X=\mathbb{C} P^{N-1}$, the open symplectic vortex equations read

$$
\begin{aligned}
\partial_{\bar{z}}^{A} Z^{i} & =0, \\
* \tilde{F}_{A c}+\tilde{e}_{c}^{2}\left(\tilde{\mu}_{c} \circ Z+\tilde{r}_{c}\right) & =0 .
\end{aligned}
$$

\footnotetext{
${ }^{48}$ For $j=1, \nu$ is taken to be $\partial \Sigma_{L}$, which is also the appropriate choice for noncompact boundaries.
}

Recall that the boundary conditions in this case are Lagrangian boundary conditions for $Z^{i}$ that map each boundary to a $U(1)^{N-1}$-invariant Lagrangian torus $T^{N-1}$ as well as $\tilde{\mu}_{c}=$ $-\tilde{r}_{c}$ and $* \tilde{F}_{A c}=0$.

The localization of supersymmetric path integrals of the form (6.24) thus reduce them to ordinary integrals of differential forms over the moduli spaces of open symplectic vortices, which are the spaces of solutions to the open symplectic vortex equations up to gauge equivalence. As we shall show in the next subsection, these moduli spaces are finite dimensional, though they may be noncompact and contain singularities. The (infinite-dimensional) path integrals thus reduce to finite-dimensional integrals, which are well-defined mathematically (modulo issues related to the aforementioned noncompactness and singularities of the moduli spaces). These finite-dimensional integrals give us numbers that can be identified with the open version of the Hamiltonian Gromov-Witten invariants of $X$ in [3-5].

We note that in the limit where $e^{2} \rightarrow+\infty$, a dynamically gauged sigma model with target $X$ flows to an ordinary sigma model with target $X / / G$ [6]. Hence, in analogy with the closed case [30], it is predicted that there is a relationship between the open Hamiltonian Gromov-Witten invariants of $X$ and the open Gromov-Witten invariants of $X / / G[16]$.

\section{Dimension of moduli space of open symplectic vortices and $\boldsymbol{R}$ anomaly}

We may attempt to compute the boundary axial $R$ anomaly of the open gauged $A$ model and find the dimension of a moduli space of open symplectic vortices. In what follows, we shall assume that we have a compact open Riemann surface, $\Sigma$, with arbitrary genus, $g$, and an arbitrary number of boundary circles, $h$. 
To evaluate the anomaly, we ought to double the open world sheet as well as the bundles on it, as in [20], in order to form a closed world sheet, on which the indices of the relevant operators can be evaluated. This is done by taking the metric on the world sheet close to each component of $\partial \Sigma$ to be that of a flat cylinder, and gluing $\Sigma$ with its orientation reversal, $\Sigma^{*}$. The resulting closed Riemann surface is denoted $\Sigma \# \Sigma^{*}$. The corresponding bundles over $\Sigma$ and $\Sigma^{*}$ are glued using the relevant boundary conditions found in Sec. V.E. The details of the evaluation can be found in [17], and we shall state only the result here. For compact $G$, the axial $R$ anomaly is

$$
\mathcal{A}=\mu\left(\phi^{*} \operatorname{ker} d \pi_{E}^{(1,0)}, \phi^{*}\left[\operatorname{ker} d \pi_{E_{L}}\right]^{(1,0)}\right)+\left(\operatorname{dim}_{\mathbb{C}}(X)+\operatorname{dim}(G)\right)(2-2 g-h),
$$

where the first term on the right-hand side is the equivariant Maslov index for the pair $(X, L)$ ( $L$ being the relevant Lagrangian submanifold). Hence, in order for correlation functions to be nonzero, an appropriate number of boundary operators whose axial $R$ charges sum to $\mathcal{A}$ should be inserted into the path integral, such that axial $R$ symmetry is preserved at the boundaries.

In addition, the virtual real dimension of the moduli spaces of open symplectic vortices (for compact $G$ ) is given by

$$
\mu\left(\phi^{*} \operatorname{ker} d \pi_{E}^{(1,0)}, \phi^{*}\left[\operatorname{ker} d \pi_{E_{L}}\right]^{(1,0)}\right)+\left(\operatorname{dim}_{\mathbb{C}}(X)-\operatorname{dim}(G)\right)(2-2 g-h) .
$$

This is obtained from the index of the linearized operator [whose index gives the dimension of the moduli spaces ([31], page 142)] one derives from the symplectic vortex equations.

\section{D. $\hat{Q}_{A}^{2} \neq 0$ anomaly}

We have previously defined open Hamiltonian GromovWitten invariants as integrals over the moduli spaces of open symplectic vortices. However, as mentioned, we have, in fact, ignored problems related to singularities in such a moduli space. In particular, we have ignored the singular boundary strata that have codimension one in the moduli space, which occur due to disk bubbling [15]. This phenomenon obstructs integration over the moduli space.

This is also a problem for ordinary open Gromov-Witten invariants, since disk bubbling also causes singular codimension one boundary strata in the moduli spaces of open world sheet instantons of the nongauged open $A$ model [32]. Disk bubbling manifests itself in the open $A$ model as a nonperturbative instanton effect, which causes the violation of the nilpotency of the scalar supercharge, i.e., $Q_{A}^{2} \neq 0$ ([20], page 833). Moreover, this anomaly of the supersymmetry algebra also spoils the cohomological structure of the space of supersymmetric ground states of the open $A$ model, which are identified with elements of the Floer cohomology group for a pair of intersecting Lagrangian submanifolds. In fact, the anomaly implies that there are no supersymmetric ground states, and therefore supersymmetry is broken.

Now, the fact that open symplectic vortices are open world sheet instantons when $G$ is trivial means that open symplectic vortices cause $Q_{A}^{2} \neq 0$ and therefore supersymmetry breaking, for trivial $G$. Thus, for nontrivial $G$, we expect that open symplectic vortices will cause an analogous effect in the open $G$-gauged $A$ model, i.e., $\hat{Q}_{A}^{2} \neq 0$ (where $\hat{Q}_{A}=Q_{A}+Q_{\mathrm{BRST}}$, with $Q_{\mathrm{BRST}}$ being the BRST charge), indicating singular codimension one boundary strata in the moduli spaces of open symplectic vortices, and implying that the space of supersymmetric ground states of the open gauged $A$ model (which we expect to be elements of the vortex Floer cohomology group $[3,33]$ for a pair of $G$-invariant Lagrangian submanifolds) would not only lose their cohomological structure, but would cease to be supersymmetric, implying supersymmetry breaking.

For the nongauged open $A$ model, it is difficult to directly compute the violation of $Q_{A}^{2}=0$ in general; one can only do so for specific examples, e.g., $X=S^{2}$ [27]. Fortunately, at least for toric manifolds with $c_{1}(X) \geq 0$, one is able to use the mirror theory, which does not contain solitonic objects, to compute this violation in general (and identify the condition whereby it vanishes) via canonical quantization, as shown by Hori [20,27]. The condition found was that for a pair of Lagrangian submanifolds supporting flat $U(1)$ bundles, the $Q_{A}^{2} \neq 0$ anomaly vanishes if and only if the values of the superpotential on the mirror B-branes match each other, and in such a case supersymmetry is manifest.

It is thus natural to investigate the $\hat{Q}_{A}^{2} \neq 0$ anomaly due to open symplectic vortices in the open gauged $A$ model via canonical quantization of its mirror theory. We shall do this for toric target spaces $X=\mathbb{C}^{N} / / U(1)^{N-k}$, i.e., by topologically A twisting the $U(1)^{k}$-GNLSM on an infinite strip whose boundaries are mapped to different equivariant A-branes in $X .^{49}$ Performing the topological A twist for the mirror theory (cf. Sec. V.D) on a Euclidean world sheet (with measure $d^{2} x=d x^{1} d x^{2}$ ) amounts to the following field redefinitions:

\footnotetext{
${ }^{49}$ These equivariant A-branes are labeled by the GLSM parameters $s_{j}^{\pi}$ and $s_{j}^{0}$, which determine the position of their respective mirror D0-branes (see footnote 39 ).
} 


$$
\begin{array}{rlrl}
\tilde{\varphi}_{c} & =-i 2 \tilde{\sigma}_{c}, & \tilde{\xi}_{c}=\overline{\tilde{\sigma}}_{c} / 4, \\
\tilde{\lambda}_{c} & =\tilde{\lambda}_{+c}, \quad \overline{\tilde{\lambda}}_{c}=\overline{\tilde{\lambda}}_{-c}, \\
\tilde{\psi}_{+c} & =\frac{2 i}{\sqrt{2}} \overline{\tilde{\lambda}}_{+c}, & \tilde{\psi}_{-c}=\frac{2 i}{\sqrt{2}} \tilde{\lambda}_{-c}, \\
\chi^{\theta c} & =\chi_{+}^{\theta c}, \quad \bar{\chi}^{\theta c}=\bar{\chi}_{-}^{\theta c}, \\
\mathcal{X}_{+}^{\theta c} & =2 \bar{\chi}_{+}^{\theta c}, \quad \mathcal{X}_{-}^{\theta c}=2 \chi_{-}^{\theta c},
\end{array}
$$

where $\tilde{\lambda}_{c}, \overline{\tilde{\lambda}}_{c}, \chi^{\theta c}$, and $\bar{\chi}^{\theta c}$ are scalars, while $\tilde{\psi}_{ \pm c}=i \tilde{\psi}_{2} \pm \tilde{\psi}_{1}$ and $\mathcal{X}_{ \pm}^{\theta c}=i \mathcal{X}_{2}^{\theta c} \pm \mathcal{X}_{1}^{\theta c}$ are one-forms. Hence, the mirror action of the open $U(1)^{k}$-gauged $A$ model with toric target $X=\mathbb{C}^{N} / / U(1)^{N-k}$ is

$$
\begin{aligned}
S_{E}= & \frac{1}{2 \pi} \int d^{2} x\left[\sum_{c}^{k} \sum_{d}^{k}\left(g_{c d} \partial_{\mu} \theta_{c} \partial^{\mu} \bar{\theta}_{d}-\frac{i}{4} g_{c d} \bar{\chi}_{c}^{\theta}\left(\stackrel{\leftrightarrow}{\partial}_{+}\right) \mathcal{X}_{-d}^{\theta}-\frac{i}{4} g_{c d} \mathcal{X}_{+c}^{\theta} \stackrel{\leftrightarrow}{\partial}_{-}\right) \chi_{d}^{\theta}-g_{c d} E_{c}^{\theta} \bar{E}_{d}^{\theta}\right) \\
& \left.+\sum_{c}^{k} \frac{1}{2 \tilde{e}_{c}^{2}}\left(\left(\tilde{F}_{12 c}\right)^{2}+i 2 \partial_{\mu} \tilde{\varphi}_{c} \partial^{\mu} \tilde{\xi}_{c}-\left(\tilde{D}_{c}\right)^{2}-\frac{\sqrt{2}}{4} \tilde{\psi}_{+c}\left(\stackrel{\leftrightarrow}{\partial}_{-}\right) \tilde{\lambda}_{c}-\frac{\sqrt{2}}{4} \overline{\tilde{\lambda}}_{c} \stackrel{\leftrightarrow}{\partial}_{+}\right) \tilde{\psi}_{-c}\right) \\
& -\frac{1}{2}\left(\sum_{j}^{N} \sum_{c}^{k} \sum_{d}^{k} \tilde{Q}_{j c} v_{d}^{j}\left(\frac{i}{2} \tilde{\varphi}_{c} E_{d}^{\theta}-\frac{\sqrt{2}}{4} \tilde{\psi}_{+c} \mathcal{X}_{-d}^{\theta}-\frac{\sqrt{2}}{4} \tilde{\psi}_{-c} \mathcal{X}_{+d}^{\theta}+\left(\tilde{D}_{c}-\tilde{F}_{12 c}\right) \theta_{d}\right)\right. \\
& +\sum_{c}^{k}\left(\sum_{j}^{N} \tilde{Q}_{j c} \hat{s}^{j}-\tilde{t}_{c}\right)\left(\tilde{D}_{c}-\tilde{F}_{12 c}\right)+\sum_{j}^{N} e^{-\sum_{c} v_{c}^{j} \theta_{c}-\hat{s}^{j}}\left(-\frac{1}{4} \sum_{c}^{k} v_{c}^{j} \mathcal{X}_{+c}^{\theta} \sum_{d}^{k} v_{d}^{j} \mathcal{X}_{-d}^{\theta}-\sum_{c}^{k} v_{c}^{j} E_{c}^{\theta}\right) \\
& +\sum_{j}^{N} \sum_{c}^{k} \sum_{d}^{k} \tilde{Q}_{j c} v_{d}^{j}\left(4 \tilde{\xi}_{c} \bar{E}_{d}^{\theta}-i \tilde{\lambda}_{c} \bar{\chi}_{d}^{\theta}-i \overline{\tilde{\lambda}}_{c} \chi_{d}^{\theta}+\left(\tilde{D}_{c}+\tilde{F}_{12 c}\right) \bar{\theta}_{d}\right) \\
& \left.\left.+\sum_{c}^{k}\left(\sum_{j}^{N} \tilde{Q}_{j c} \overline{\hat{s}}^{j}-\overline{\tilde{t}}_{c}\right)\left(\tilde{D}_{c}+\tilde{F}_{12 c}\right)+\sum_{j}^{N} e^{-\sum_{c} v_{c}^{j} \bar{\theta}_{c}-\hat{s}^{j}}\left(-\sum_{c}^{k} v_{c}^{j} \bar{\chi}_{c}^{\theta} \sum_{d}^{k} v_{d}^{j} \chi_{d}^{\theta}-\sum_{c}^{k} v_{c}^{j} \bar{E}_{c}^{\theta}\right)\right)\right]
\end{aligned}
$$

(where $\partial_{ \pm}=i \partial_{2} \pm \partial_{1}$ ), which is invariant under the supersymmetry transformations

$$
\begin{aligned}
\delta_{Q_{A}} \tilde{A}_{1 c} & =\frac{\sqrt{2}}{2} \epsilon \tilde{\psi}_{1 c}, \quad \delta_{Q_{A}} \tilde{\psi}_{-c}=-\frac{1}{\sqrt{2}} \epsilon \partial_{-} \tilde{\varphi}_{c}, \\
\delta_{Q_{A}} \tilde{A}_{2 c} & =\frac{\sqrt{2}}{2} \epsilon \tilde{\psi}_{2 c}, \quad \delta_{Q_{A}} \tilde{\psi}_{+c}=-\frac{1}{\sqrt{2}} \epsilon \partial_{+} \tilde{\varphi}_{c}, \\
\delta_{Q_{A}} \tilde{\varphi}_{c} & =0, \quad \delta_{Q_{A}} \overline{\tilde{\lambda}}_{c}=-i \epsilon\left(\tilde{F}_{12 c}+\tilde{D}_{c}\right), \\
\delta_{Q_{A}} \tilde{\xi}_{c} & =-\frac{i \epsilon\left(\overline{\tilde{\lambda}}_{c}+\tilde{\lambda}_{c}\right)}{4}, \quad \delta_{Q_{A}} \tilde{\lambda}_{c}=i \epsilon\left(\tilde{F}_{12 c}+\tilde{D}_{c}\right), \\
\delta_{Q_{A}} \tilde{D}_{c} & =-\frac{\sqrt{2}}{2} \epsilon\left(\partial_{1} \tilde{\psi}_{2 c}-\partial_{2} \tilde{\psi}_{1 c}\right), \quad \delta_{Q_{A}} \mathcal{X}_{-}^{\theta c}=-2 i \epsilon \partial_{-} \theta^{c}, \\
\delta_{Q_{A}} \theta^{c} & =0, \quad \delta_{Q_{A}} \mathcal{X}_{+}^{\theta c}=2 i \epsilon \partial_{+} \theta^{c}, \\
\delta_{Q_{A}} \bar{\theta}^{c} & =\epsilon\left(\chi^{\theta c}-\bar{\chi}^{\theta c}\right), \quad \delta_{Q_{A}} \bar{\chi}^{\theta c}=\epsilon \bar{E}^{\theta c}, \\
\delta_{Q_{A}} E^{\theta c} & =i \epsilon \partial^{\mu} \mathcal{X}_{\mu}^{\theta c}, \quad \delta_{Q_{A}} \chi^{\theta c}=\epsilon \bar{E}^{\theta c}, \\
\delta_{Q_{A}} \bar{E}^{\theta c} & =0, \quad
\end{aligned}
$$

generated by the supercharge $Q_{A}$. However, this mirror theory is, in fact, a gauge theory, and any consistent quantization procedure should include gauge fixing, in order to remove unphysical degrees of freedom. 
To this end, we shall choose the Lorentz gauge

$$
\left\langle\psi^{\prime}\left|\partial_{\mu} \tilde{A}_{c}^{\mu}\right| \psi\right\rangle=0
$$

(where $|\psi\rangle$ and $\left|\psi^{\prime}\right\rangle$ are physical states), which is effected by including the following BRST gauge fixing action:

$$
\begin{aligned}
S_{\mathrm{BRST}}= & \frac{1}{2 \pi} \sum_{c}^{k} \frac{1}{2 \tilde{e}_{c}^{2}} \int d^{2} x\left(-i B_{c} \partial_{\mu} \tilde{A}_{c}^{\mu}-\left(B_{c}\right)^{2}\right. \\
& \left.+\partial_{\mu} \tilde{b}_{c} \partial^{\mu} \tilde{c}_{c}-i \frac{\sqrt{2}}{2} \partial_{\mu} \tilde{b}_{c} \tilde{\psi}_{c}^{\mu}\right),
\end{aligned}
$$

where $\tilde{b}_{c}$ and $\tilde{c}_{c}$ are fermionic ghost fields, while $B_{c}$ is a bosonic auxiliary field. As expected, the first term explicitly breaks the $U(1)^{k}$ gauge symmetry of the gauged LG model.

Now, note that the gauge fixing action (6.33) can be rewritten as

$$
\begin{aligned}
S_{\mathrm{BRST}}= & \epsilon^{-1}\left(\delta_{Q_{A}}+\delta_{\mathrm{BRST}}\right) \\
& \times\left\{\sum_{c}^{k} \frac{1}{2 \tilde{e}_{c}^{2}} \int d^{2} x\left(-i \tilde{b}_{c} \partial_{\mu} \tilde{A}_{c}^{\mu}+\tilde{b}_{c} B_{c}\right)\right\},
\end{aligned}
$$

where we have performed integration by parts and used the boundary condition $\left(\tilde{\lambda}_{-c}-\overline{\tilde{\lambda}}_{+c}\right)=0$ (which is equivalent to $\tilde{\psi}_{1 c}=0$ ) that we have previously imposed, as well as the boundary condition

$$
\partial_{1} \tilde{c}_{c}=0
$$

which we impose at present. Here, $\delta_{\mathrm{BRST}}$ is the standard BRST symmetry variation given by

$$
\begin{aligned}
\delta_{\mathrm{BRST}} \tilde{A}_{\mu c} & =i \epsilon \partial_{\mu} \tilde{c}_{c}, \\
\delta_{\mathrm{BRST}} \tilde{b}_{c} & =\epsilon B_{c}, \\
\delta_{\mathrm{BRST}} \tilde{c}_{c} & =0, \\
\delta_{\mathrm{BRST}} B_{c} & =0,
\end{aligned}
$$

with the BRST variations of all other fields being equal to zero. For the unphysical fields used for gauge fixing, the supersymmetry transformations are $\delta_{Q_{A}} \tilde{b}_{c}=0$ and $\delta_{Q_{A}} B_{c}=0$ while ${ }^{50}$

$$
\delta_{Q_{A}} \tilde{c}_{c}=-\frac{i}{2} \epsilon \tilde{\varphi}_{c}
$$

\footnotetext{
${ }^{50}$ Note that with respect to (6.37), the boundary condition (6.35) obeys $A$-type supersymmetry, since the boundary condition on $\tilde{\varphi}_{c}$ is $\partial_{1} \tilde{\varphi}_{c}=0$.
}

Now, $\delta_{Q_{A}}^{2} \propto \delta_{G}(\tilde{\varphi})$ and $\delta_{\mathrm{BRST}}^{2}=0$ on all fields. ${ }^{51}$ In addition, we can show that

$$
\left(\delta_{Q_{A}}+\delta_{\mathrm{BRST}}\right)^{2}=0
$$

on all fields. This implies that the BRST gauge fixing action (6.34) is, in fact, invariant under $\hat{\delta}=\delta_{Q_{A}}+\delta_{\mathrm{BRST}}$. Since the physical action (6.30) is also invariant under $\delta_{\mathrm{BRST}}$, this further implies that the entire action $S_{E}+S_{\mathrm{BRST}}$ is invariant under $\hat{\delta}=\delta_{Q_{A}}+\delta_{\text {BRST }}$. This suggests that the relevant symmetry of the action after gauge fixing is that which is generated by $\hat{Q}_{A}=Q_{A}+Q_{\text {BRST. }}$.

The nonvanishing canonical commutation and anticommutation relations are

$$
\begin{aligned}
{\left[\theta^{c}\left(x^{1}\right), g_{d e} \partial_{2} \bar{\theta}^{e}\left(y^{1}\right)\right] } & =2 \pi \delta_{d}^{c} \delta\left(x^{1}-y^{1}\right), \\
{\left[\bar{\theta}^{c}\left(x^{1}\right), g_{d e} \partial_{2} \theta^{e}\left(y^{1}\right)\right] } & =2 \pi \delta_{d}^{c} \delta\left(x^{1}-y^{1}\right), \\
\left\{\chi^{\theta c}\left(x^{1}\right), \mathcal{X}_{+}^{\theta d}\left(y^{1}\right)\right\} & =4 \pi g^{c d} \delta\left(x^{1}-y^{1}\right), \\
\left\{\mathcal{X}_{-}^{\theta c}\left(x^{1}\right), \bar{\chi}^{\theta d}\left(y^{1}\right)\right\} & =4 \pi g^{c d} \delta\left(x^{1}-y^{1}\right), \\
\left.\tilde{\varphi}_{c}\left(x^{1}\right), \frac{1}{2 \tilde{e}_{d}^{2}} i 2 \partial_{2} \tilde{\xi}_{d}\left(y^{1}\right)\right] & =2 \pi \delta_{c d} \delta\left(x^{1}-y^{1}\right), \\
{\left[\tilde{\xi}_{c}\left(x^{1}\right), \frac{1}{2 \tilde{e}_{d}^{2}} i 2 \partial_{2} \tilde{\varphi}_{d}\left(y^{1}\right)\right] } & =2 \pi \delta_{c d} \delta\left(x^{1}-y^{1}\right), \\
\left\{\tilde{\lambda}_{c}\left(x^{1}\right), \frac{1}{2 \tilde{e}_{d}^{2}} \frac{\sqrt{2}}{2 i} \tilde{\psi}_{+d}\left(y^{1}\right)\right\} & =2 \pi \delta_{c d} \delta\left(x^{1}-y^{1}\right), \\
\left\{\tilde{\psi}_{-c}\left(x^{1}\right), \frac{1}{2 \tilde{e}_{d}^{2}} \frac{\sqrt{2}}{2 i} \overline{\tilde{\lambda}}_{d}\left(y^{1}\right)\right\} & =2 \pi \delta_{c d} \delta\left(x^{1}-y^{1}\right), \\
{\left[\tilde{A}_{1 c}\left(x^{1}\right),-\left(\frac{1}{\tilde{e}_{c}^{2}} \tilde{F}_{12 c}\left(y^{1}\right)\right)\right] } & =2 \pi \delta_{c d} \delta\left(x^{1}-y^{1}\right), \\
{\left[\tilde{A}_{2 c}\left(x^{1}\right), \frac{1}{2 \tilde{e}_{d}^{2}}\left(-i B_{d}\left(y^{1}\right)\right)\right] } & =2 \pi \delta_{c d} \delta\left(x^{1}-y^{1}\right), \\
\left\{\tilde{b}_{c}\left(x^{1}\right), \frac{1}{2 \tilde{e}_{d}^{2}}\left(\partial_{2} \tilde{c}_{d}\left(y^{1}\right)\right)\right\} & =2 \pi \delta_{c d} \delta\left(x^{1}-y^{1}\right), \\
\left\{\tilde{c}_{c}\left(x^{1}\right), \frac{1}{2 \tilde{e}_{d}^{2}}\left(\partial_{2} \tilde{b}_{d}\left(y^{1}\right)\right)\right\} & =2 \pi \delta_{c d} \delta\left(x^{1}-y^{1}\right),
\end{aligned}
$$

The explicit form of the supercharge $\hat{Q}_{A}$ is ${ }^{52}$

\footnotetext{
${ }^{51}$ The transformation $\delta_{G}(\tilde{\varphi})$ is a $U(1)^{k}$ gauge transformation whose local parameter is $\tilde{\varphi}_{c}$.

${ }^{52}$ Charge conservation follows from $\partial_{2} \hat{Q}_{A}=\int d x^{1} \partial_{2} \hat{J}_{A}^{2}=$ $-\int d x^{1} \partial_{1} \hat{J}_{A}^{1}$, which can be shown to be zero using the boundary conditions we have previously imposed on the fields, as well as the boundary condition $\partial_{1} \tilde{b}_{c}=0$.
} 


$$
\begin{aligned}
\hat{Q}_{A}= & \frac{1}{2 \pi} \int d x^{1}\left(g_{c d} \partial_{2} \theta^{c}\left(\chi^{\theta d}-\bar{\chi}^{\theta d}\right)-i g_{c d} \partial_{1} \theta^{c}\left(\chi^{\theta d}+\bar{\chi}^{\theta d}\right)-\sum_{c}^{k} \frac{\sqrt{2}}{4 \tilde{e}_{c}^{2}} \tilde{F}_{12 c}\left(\tilde{\psi}_{+c}-\tilde{\psi}_{-c}\right)\right. \\
& -\sum_{c}^{k} \frac{i}{\tilde{e}_{c}^{2}} \tilde{F}_{12 c} \partial_{1} \tilde{c}_{c}+\sum_{c}^{k} \frac{1}{4 \tilde{e}_{c}^{2}}\left(\tilde{\lambda}_{c}+\overline{\tilde{\lambda}}_{c}\right) \partial_{2} \tilde{\varphi}_{c}+\sum_{c}^{k} \frac{i}{4 \tilde{e}_{c}^{2}}\left(\overline{\tilde{\lambda}}_{c}-\tilde{\lambda}_{c}\right) \partial_{1} \tilde{\varphi}_{c} \\
& +\frac{1}{4} \sum_{c}^{k}\left(\mathcal{X}_{+}^{\theta c}+\mathcal{X}_{-}^{\theta c}\right) \frac{\partial \tilde{W}}{\partial \theta^{c}}+\sum_{c}^{k} \frac{\sqrt{2}}{4}\left(\tilde{\psi}_{+c}-\tilde{\psi}_{-c}\right) \frac{1}{2}\left(\frac{2}{i} \frac{\partial \tilde{W}}{\partial \tilde{\varphi}_{c}}+\frac{1}{4} \frac{\partial \tilde{\tilde{W}}}{\partial \tilde{\xi}_{c}}\right) \\
& +\sum_{c}^{k} \partial_{1} \tilde{c}_{c} \frac{1}{2 i}\left(\frac{2}{i} \frac{\partial \tilde{W}}{\partial \tilde{\varphi}_{c}}-\frac{1}{4} \frac{\partial \overline{\tilde{W}}}{\partial \tilde{\xi}_{c}}\right) \\
& \left.-\sum_{c}^{k} \frac{1}{2 \tilde{e}_{c}^{2}} \frac{\sqrt{2}}{4} B_{c}\left(\tilde{\psi}_{+c}+\tilde{\psi}_{-c}\right)+\sum_{c}^{k} \frac{1}{2 \tilde{e}_{c}^{2}} B_{c} \partial_{2} \tilde{c}_{c}+\sum_{c}^{k} \frac{i}{4 \tilde{e}_{c}^{2}} \partial_{2} \tilde{b}_{c} \tilde{\varphi}_{c}\right),
\end{aligned}
$$

where

$$
\begin{aligned}
\frac{\partial \tilde{W}}{\partial \theta^{c}} & =\frac{i}{2} \sum_{j}^{N}\left\langle\tilde{\varphi}, \tilde{Q}_{j}\right\rangle v_{c}^{j}-\sum_{j=1}^{N} v_{c}^{j} e^{-\left\langle v^{j}, \theta\right\rangle-\hat{s}^{j}}, \\
\frac{2}{i} \frac{\partial \tilde{W}}{\partial \tilde{\varphi}_{c}} & =\sum_{j}^{N} \tilde{Q}_{j c}\left(\left\langle v^{j}, \theta\right\rangle+\hat{s}^{j}\right)-\tilde{t}_{c}, \\
\frac{1}{4} \frac{\partial \tilde{\tilde{W}}}{\partial \tilde{\xi}_{c}} & =\sum_{j}^{N} \tilde{Q}_{j c}\left(\left\langle v^{j}, \bar{\theta}\right\rangle+\overline{\hat{s}}^{j}\right)-\overline{\tilde{t}}_{c} .
\end{aligned}
$$

Then,

$$
\begin{aligned}
\hat{Q}_{A}^{2}=\frac{1}{2}\left\{\hat{Q}_{A}, \hat{Q}_{A}\right\}= & \frac{1}{2 \pi} \int d x^{1}\left((-i) \sum_{c}^{k} \partial_{1} \theta^{c} \frac{\partial \tilde{W}}{\partial \theta^{c}}+(-i) \sum_{c}^{k} \partial_{1} \tilde{\varphi}_{c} \frac{\partial \tilde{W}}{\partial \tilde{\varphi}_{c}}-\sum_{c}^{k}\left(\frac{i}{4 \tilde{e}_{c}^{2}}\right) \partial_{2} \tilde{\varphi}_{c} B_{c}\right. \\
& \left.-\sum_{c}^{k} \sum_{d}^{k} \frac{\partial^{2} \overline{\tilde{W}}}{\partial \bar{\theta}^{d} \partial \tilde{\xi}_{c}}\left(\chi^{\theta d}-\bar{\chi}^{\theta d}\right)\left(\frac{i}{8}\right)\left(\frac{\sqrt{2}}{4 i}\left(\tilde{\psi}_{+c}-\tilde{\psi}_{-c}\right)+\partial_{1} \tilde{c}_{c}\right)\right),
\end{aligned}
$$

where we have used the boundary conditions $\tilde{F}_{12 c}=0$ and $\sum_{c=1}^{k} v_{c j} \Theta_{c}=s_{j}-\hat{s}_{j}$ (where $s_{j}=s_{j}^{\pi}$ at $x^{1}=\pi$ and $s_{j}=s_{j}^{0}$ at $\left.x^{1}=0\right)$, as well as the constraint $\sum_{j}^{N} \tilde{Q}_{j c} s_{j}-\tilde{t}_{c}=0$.

The terms with first-order derivatives of the superpotential can be written as

$$
\begin{aligned}
& \frac{(-i)}{2 \pi} \int d x^{1}\left(\sum_{c}^{k} \partial_{1} \theta^{c} \frac{\partial \tilde{W}}{\partial \theta^{c}}+\sum_{c}^{k} \partial_{1} \tilde{\varphi}_{c} \frac{\partial \tilde{W}}{\partial \tilde{\varphi}_{c}}\right) \\
& =\frac{(-i)}{2 \pi} \int d x^{1} \partial_{1} \tilde{W}(\theta, \tilde{\varphi}) \\
& =\frac{(-i)}{2 \pi}\left(\tilde{W}(\theta, \tilde{\varphi})_{\pi}-\tilde{W}(\theta, \tilde{\varphi})_{0}\right) .
\end{aligned}
$$

From the analysis below (5.63), we know that this is equal to $\frac{(-i)}{2 \pi}\left(\sum_{i=1}^{N} e^{-s_{i}^{\pi}}-\sum_{i=1}^{N} e^{-s_{i}^{0}}\right)$.

The remaining terms to consider are then

$$
\frac{1}{2 \pi} \int d x^{1}\left(-\sum_{c}^{k}\left(\frac{i}{4 \tilde{e}_{c}^{2}}\right) \partial_{2} \tilde{\varphi}_{c} B_{c}-\sum_{c}^{k} \sum_{d}^{k} \frac{\partial^{2} \overline{\tilde{W}}}{\partial \bar{\theta}^{d} \partial \tilde{\xi}_{c}}\left(\chi^{\theta d}-\bar{\chi}^{\theta d}\right)\left(\frac{i}{8}\right)\left(\frac{\sqrt{2}}{4 i}\left(\tilde{\psi}_{+c}-\tilde{\psi}_{-c}\right)+\partial_{1} \tilde{c}_{c}\right)\right)
$$


Unlike (6.43), these cannot be written in terms of boundary data, and hence are bulk terms that occur even for closed world sheets. However, as in the nonanomalous closed case, these bulk terms ought to be equal to zero. The vanishing of these terms can also be understood as follows. The auxiliary field $B_{c}$ obeys its equation of motion $B_{c}=-\frac{i}{2} \partial_{\mu} \tilde{A}_{c}^{\mu}$ as an operator equation due to Ehrenfest's theorem, and hence the matrix elements of the first term in the integrand with respect to the physical Hilbert space vanish due to the Lorentz gauge condition (6.32). ${ }^{53}$ Next, note that we are dealing with an A-twisted theory, whose topological correlation functions are invariant under $\hat{Q}_{A}$-exact deformations of the action. Therefore, we should be able to deform the action such that the second term in (6.44) vanishes. Indeed, this can be achieved by adding the following term that is $\hat{Q}_{A}$ exact to the action, i.e.,

$$
\begin{aligned}
\epsilon^{-1} \delta_{\hat{Q}_{A}} & {\left[\frac{1}{2 \pi} \int d^{2} x \frac{1}{2}\left(\sum_{c}^{k} i \tilde{\lambda}_{c}\left(\sum_{j}^{N} \tilde{Q}_{j c}\left(\sum_{d} v_{d}^{j} \bar{\theta}^{d}+\overline{\hat{s}}^{j}\right)-\overline{\tilde{t}}_{c}\right)+\sum_{c}^{k} 4 \tilde{\xi}_{c}\left(\sum_{j}^{N} \sum_{d}^{k} \tilde{Q}_{j c} v_{d}^{j} \bar{\chi}_{d}\right)\right)\right] } \\
= & \frac{1}{2 \pi} \int d^{2} x \frac{1}{2}\left(\sum_{j}^{N} \sum_{c}^{k} \sum_{d}^{k} \tilde{Q}_{j c} v_{d}^{j}\left(4 \tilde{\xi}_{c} \bar{E}_{d}^{\theta}-i \tilde{\lambda}_{c} \bar{\chi}_{d}^{\theta}-i \overline{\tilde{\lambda}}_{c} \chi_{d}^{\theta}+\left(\tilde{D}_{c}+\tilde{F}_{12 c}\right) \bar{\theta}_{d}\right)\right. \\
& \left.+\sum_{c}^{k}\left(\sum_{j}^{N} \tilde{Q}_{j c} \overline{\hat{s}}^{j}-\overline{\tilde{t}}_{c}\right)\left(\tilde{D}_{c}+\tilde{F}_{12 c}\right)\right),
\end{aligned}
$$

which upon doing so, the terms proportional to $\partial \overline{\tilde{W}}(\bar{\theta}, \tilde{\xi}) / \partial \tilde{\xi}_{c}$ in (6.40) vanish, whence the second term in (6.44) also vanishes.

Hence, we find that

$$
\hat{Q}_{A}^{2}=\frac{(-i)}{2 \pi}\left(\tilde{W}(\theta, \tilde{\varphi})_{\pi}-\tilde{W}(\theta, \tilde{\varphi})_{0}\right)
$$

i.e., the $\hat{Q}_{A}^{2} \neq 0$ anomaly (which occurs due to the nonperturbative quantum effects of open symplectic vortices in the open gauged $A$ model) vanishes when the value of the superpotential $\tilde{W}(\theta, \tilde{\varphi})$ is equal on both boundaries, i.e., $\sum_{i=1}^{N} e^{-s_{i}^{\pi}}=\sum_{i=1}^{N} e^{-s_{i}^{0}}$. In other words, there is no anomaly when each boundary ends on a D0-brane such that both D0-branes are mapped to the same value of $\tilde{W}(\theta, \tilde{\varphi})$. One way this can occur is when the boundaries end on coincident D0-branes. Although the condition $\sum_{i=1}^{N} e^{-s_{i}^{\pi}}=\sum_{i=1}^{N} e^{-s_{i}^{0}}$ seems identical to the condition (found by Hori [20,27]) for the vanishing of the $Q_{A}^{2} \neq 0$ anomaly of the open $A$ model, the D0-branes do not have to be located at a critical point where $\partial_{\theta_{c}} \tilde{W}_{X}=0$ in our case (where $\tilde{W}_{X}$ is the superpotential in the nongauged case, which depends only on $\theta$ in the bulk), and the position of each D0-brane [defined by $s_{i}$ via (5.62)] is in our case constrained by $\sum_{i}^{N} \mathcal{Q}_{a}^{i} s_{i}-t_{a}=0$ instead of just

\footnotetext{
${ }^{53}$ This statement follows from the fact that the Lorentz gauge condition can equivalently be written as $\partial_{\mu}\left(\tilde{A}_{c}^{\mu}\right)^{+}|\psi\rangle=0$ or $\langle\psi| \partial_{\mu}\left(\tilde{A}_{c}^{\mu}\right)^{-}=0$ [where $\tilde{A}_{c}^{\mu}=\left(\tilde{A}_{c}^{\mu}\right)^{+}+\left(\tilde{A}_{c}^{\mu}\right)^{-}$is the decomposition with respect to positive and negative momenta], as well as the fact that $\partial_{\mu} \tilde{A}_{c}^{\mu}$ commutes with $\partial_{2} \tilde{\varphi}_{c}$.
}

$\sum_{i}^{N} \hat{Q}_{b}^{i} s_{i}-\hat{t}_{b}=0$. In conclusion, for Abelian $G$, we have found that for a pair of $G$-invariant Lagrangian tori of a toric manifold supporting flat $G$-equivariant $U(1)$ bundles, the quantum anomaly of $\hat{Q}_{A}^{2} \neq 0$ (which indicates an obstruction to integration over the moduli spaces of open symplectic vortices) vanishes if and only if the values of the superpotential $\tilde{W}(\theta, \tilde{\varphi})$ on the mirror B-branes are the same, and in this case, supersymmetry is manifest.

\section{E. Mirror computation of Abelian invariants}

In principle, it is simpler to use the mirror gauged Landau-Ginzburg description of the open gauged $A$ model to compute open Hamiltonian Gromov-Witten invariants for Abelian gauge groups and toric target spaces with $c_{1}(X) \geq 0$, since there are no open symplectic vortices in this gauged LG model.

We shall focus on the mirror computation of invariants that come from path integrals over the $Q_{A}$-invariant local observables associated with equivariant cohomology classes, i.e., those given by (6.11) (where $d_{G} \alpha=0$ ) and (6.22) (where $\left.d_{G} \zeta=0\right)$. ${ }^{54}$ After integrating out the auxiliary fields, the supersymmetry transformations (generated by $\hat{Q}_{A}$ ) of the physical fields of the mirror theory on a Euclidean world sheet parametrized by complex coordinates $(z, \bar{z})$ are (with $\epsilon=\sqrt{2})^{55}$

\footnotetext{
${ }^{54}$ Note that the $G$ invariance of these physical observables implies that they are invariant under $Q_{\mathrm{BRST}}$, and therefore also invariant under $\hat{Q}_{A}$.

${ }^{55}$ The fermionic fields $\tilde{\eta}_{c}$ and $\tilde{\kappa}_{c}$ are each linear combinations of the fields $\tilde{\lambda}_{c}$ and $\overline{\tilde{\lambda}}_{c}$ defined in the previous section.
} 


$$
\begin{aligned}
\delta_{\hat{Q}_{A}} \tilde{A}_{z c} & =\tilde{\psi}_{z c}+i \sqrt{2} \partial_{z} \tilde{c}_{c}, \quad \delta_{\hat{Q}_{A}} \tilde{\psi}_{z c}=-\partial_{z} \tilde{\varphi}_{c}, \\
\delta_{\hat{Q}_{A}} \tilde{A}_{\bar{z} c} & =\tilde{\psi}_{\bar{z} c}+i \sqrt{2} \partial_{\bar{z}} \tilde{c}_{c}, \quad \delta_{\hat{Q}_{A}} \tilde{\psi}_{\bar{z} c}=-\partial_{\bar{z}} \tilde{\varphi}_{c}, \\
\delta_{\hat{Q}_{A}} \tilde{\varphi}_{c} & =0, \quad \delta_{\hat{Q}_{A}} \tilde{\eta}_{c}=0, \\
\delta_{\hat{Q}_{A}} \tilde{\xi}_{c} & =\tilde{\eta}_{c}, \quad \delta_{\hat{Q}_{A}} \tilde{\kappa}_{c}=2\left[* \tilde{F}_{A c}-\tilde{e}_{c}^{2}\left(-i \partial_{\tilde{\varphi}_{c}} \tilde{W}+(1 / 8) \partial_{\tilde{\xi}_{c}} \overline{\tilde{W}}\right)\right], \\
\delta_{\hat{Q}_{A}} \theta^{c} & =0, \quad \delta_{\hat{Q}_{A}} \mathcal{X}_{z}^{\theta c}=-2 \sqrt{2} i \partial_{z} \theta^{c}, \\
\delta_{\hat{Q}_{A}} \bar{\theta}^{c} & =\sqrt{2}\left(\chi^{\theta c}-\bar{\chi}^{\theta c}\right), \quad \delta_{\hat{Q}_{A}} \mathcal{X}_{\bar{z}}^{\theta c}=2 \sqrt{2} i \partial_{\bar{z}} \theta^{c}, \\
\delta_{\hat{Q}_{A}}\left(\chi^{\theta c}-\bar{\chi}^{\theta c}\right) & =0, \quad \delta_{\hat{Q}_{A}}\left[g_{c d}\left(\chi^{\theta d}+\bar{\chi}^{\theta d}\right)\right]=-\sqrt{2} \partial_{\theta^{c}} \tilde{W} .
\end{aligned}
$$

The bulk physical operators of this theory were studied by Baptista [7], where he showed that the bulk chiral ring is given by

$$
\mathbb{C}\left[\tilde{\varphi}^{1}, \ldots, \tilde{\varphi}^{k},\left(x^{1}\right)^{ \pm 1}, \ldots,\left(x^{k}\right)^{ \pm 1}\right] / D(\tilde{W}),
$$

i.e., holomorphic functions of $\tilde{\varphi}_{c}$ and $\left(x^{c}\right)^{ \pm 1}:=\exp \left(\mp \theta^{c}\right)$, modulo the ideal $D(\tilde{W})$, where $D(\tilde{W})$ is generated by the derivatives

$$
\partial_{\theta^{c}} \tilde{W}=-x^{c} \partial_{x^{c}} \tilde{W}=\sum_{j=1}^{n} \tilde{Q}_{j}^{c}\left[\frac{i}{2}\left\langle\tilde{\varphi}, v^{j}\right\rangle-e^{-\hat{s}^{j}} \prod_{d=1}^{k}\left(x^{d}\right)^{v_{d}^{j}}\right] .
$$

In addition, one ought to restrict the bulk physical operators to finite-degree polynomials, since in the equivariant de Rham complex one considers only finite-degree forms and polynomials in the Lie algebra.

Aided by the boundary conditions and (6.47), it can be deduced that the boundary chiral ring at a particular boundary component $\partial \Sigma_{L}$ is given by

$$
\mathbb{C}\left[\tilde{\varphi}^{1}, \ldots, \tilde{\varphi}^{k}\right] /\left(\left.D(\tilde{W})\right|_{\partial \Sigma_{L}}\right) .
$$

Here, we have taken into account the fact that $\partial_{\theta^{c}} \tilde{W}$ is a $\hat{Q}_{A}$-exact function of $\tilde{\varphi}_{c}$ at the boundaries. Moreover, we ought to restrict the boundary physical operators to finite-degree polynomials, as we did for the bulk physical operators. Note that in the nonequivariant case, the critical point condition $\partial_{\theta_{c}} \tilde{W}=0$ at the boundaries implies that the $k$ fermionic fields $\chi^{\theta c}+\bar{\chi}^{\theta c}$ are $Q_{A}$ invariant at each boundary and, in fact, form the boundary chiral ring [10]. However, recall from Sec. V. D that we do not have such a critical point condition, implying that $\chi^{\theta c}+\bar{\chi}^{\theta c}$ is not $\hat{Q}_{A}$ invariant at the boundaries, and therefore is not an element of the boundary chiral ring (6.50).

Denoting an arbitrary element of the bulk chiral ring (6.48) as $W^{\text {mirror }}$, and an arbitrary element of a boundary chiral ring (6.50) as $W_{\partial \Sigma_{L}}^{\text {mirror }}$, the most general correlation function of local bulk and boundary observables in the gauged LandauGinzburg model is therefore written (for $\Sigma=I \times \mathbb{R}$ ) as

$$
\int \mathcal{D}\left(\tilde{A}, \theta, \tilde{\varphi}, \tilde{\xi}, \tilde{\eta}, \tilde{\kappa}, \tilde{\psi}, \chi^{\theta}, \tilde{b}, \tilde{c}\right) e^{-\left(S_{A}+S_{\mathrm{BRST}}\right)} \prod_{i} W_{i}^{\text {mirror }} \prod_{j} W_{\partial \Sigma_{0} \mid j}^{\text {mirror }} \prod_{k} W_{\partial \Sigma_{\pi} \mid k}^{\text {mirror }}
$$

This is the mirror correlation function that computes (6.24) for local observables.

\section{ACKNOWLEDGMENTS}

We would like to thank Sushmita Venugopalan for explaining to us many relevant mathematical points regarding the moduli space of open symplectic vortices. We would also like to thank João Manuel Baptista, Sebastian Goette, Suresh Govindarajan, Yuan Luo, Daniel S. Park, Petr Vaśko, Junya Yagi, Masaya Yata, and Qin Zhao for helpful comments and discussions. This work is supported by NUS Tier 1 FRC Grant No. R-144-000-316-112. 
[1] J. Polchinski, Dirichlet Branes and Ramond-Ramond Charges, Phys. Rev. Lett. 75, 4724 (1995).

[2] M. Kontsevich, Homological algebra of mirror symmetry, in Proceedings of the International Congress of Mathematicians, Zurich, 1994 (Birkhauser, Boston, 1995), pp. 120-139.

[3] K. Cieliebak, A. R. Gaio, and D. A. Salamon, J-holomorphic curves, moment maps, and invariants of Hamiltonian group actions, Int. Math. Res. Not. 16, 831 (2000).

[4] I. Mundet i Riera, Hamiltonian Gromov-Witten invariants, Topology 42, 525 (2003).

[5] K. Cieliebak, A. R. Gaio, I. Mundet i Riera, and D. A. Salamon, The symplectic vortex equations and invariants of Hamiltonian group actions, J. Symplectic Geom. 1, 543 (2002).

[6] J. M. Baptista, Twisting gauged non-linear sigma-models, J. High Energy Phys. 02 (2008) 096.

[7] J. M. Baptista, The quantum equivariant cohomology of toric manifolds through mirror symmetry, J. High Energy Phys. 04 (2009) 017.

[8] K. Hori and C. Vafa, Mirror symmetry, arXiv:hep-th/ 0002222.

[9] K. Hori, A. Iqbal, and C. Vafa, D-branes and mirror symmetry, arXiv:hep-th/0005247.

[10] K. Hori, Linear models of supersymmetric D-branes, in Symplectic Geometry and Mirror Symmetry, Seoul, 2000, edited by K. Fukaya et al. (World Scientific, River Edge, NJ, 2001), pp. 111-186.

[11] A. Kapustin, K. Setter, and K. Vyas, Surface operators in four-dimensional topological gauge theory and Langlands duality, arXiv:1002.0385.

[12] K. Setter, Doctoral dissertation, California Institute of Technology, 2013.

[13] B. Fang, C.-C. M. Liu, D. Treumann, and E. Zaslow, T-duality and homological mirror symmetry for toric varieties, Adv. Math. 229, 1873 (2012).

[14] M. Futaki and K. Ueda, Tropical coamoeba and torusequivariant homological mirror symmetry for the projective space, Commun. Math. Phys. 332, 53 (2014).

[15] G. Xu, The moduli space of twisted holomorphic maps with Lagrangian boundary condition: Compactness, Adv. Math. 242, 1 (2013).

[16] D. Wang and G. Xu, Compactness in the adiabatic limit of disk vortices, arXiv:1505.05945.

[17] M. Ashwinkumar and M.-C. Tan, Open gauged sigma models, equivariant branes and equivariant homological mirror symmetry, arXiv:1612.09191.
[18] E. Witten, Phases of $N=2$ theories in two dimensions, Nucl. Phys. B403, 159 (1993).

[19] S. Govindarajan, T. Jayaraman, and T. Sarkar, On D-branes from gauged linear sigma models, Nucl. Phys. B593, 155 (2001).

[20] K. Hori et al., Mirror Symmetry, Clay Mathematics Monographs 1 (AMS, Providence, RI, 2003).

[21] K. Vyas, Doctoral dissertation, California Institute of Technology, 2010.

[22] R. J. Szabo, Equivariant Cohomology and Localization of Path Integrals, Springer Science and Business Media (Springer, New York, 2003).

[23] N. Berline, E. Getzler, and M. Vergne, Heat Kernels and Dirac Operators, Springer Science and Business Media (Springer, New York, 1992).

[24] P. S. Aspinwall, D-branes on Calabi-Yau manifolds, in Progress in String Theory: TASI 2003, edited by J. Maldacena (World Scientific, Singapore, 2005), pp. 1-152.

[25] S. Cautis and J Kamnitzer, Knot homology via derived categories of coherent sheaves, I: The $\mathfrak{s l}(2)$-case, Duke Math. J. 142, 511 (2008).

[26] C.H. Cho and H. Hong, Finite group actions on Lagrangian Floer theory, J. Symplectic Geom. 15, 307 (2017).

[27] K. Hori, Mirror symmetry and quantum geometry, in Proceedings of ICM 2002, Vol. III, edited by T. Li (Higher Education Press, Beijing, 2002), pp. 431-443.

[28] A. Kapustin and Y. Li, D-branes in Landau-Ginzburg models and algebraic geometry, J. High Energy Phys. 12 (2003) 005.

[29] J. M. Figueroa-O'Farrill and S. Stanciu, Equivariant cohomology and gauged bosonic sigma-models, arXiv:hep-th/ 9407149.

[30] A. R. Gaio and D. A. Salamon, Gromov-Witten invariants of symplectic quotients and adiabatic limits, J. Symplectic Geom. 3, 55 (2005).

[31] D. McDuff and D. A. Salamon, Introduction to Symplectic Topology (Oxford University Press, Oxford, UK, 1998).

[32] P. V. Georgieva, Doctoral dissertation, Stanford University, 2011.

[33] G. Xu, Gauged Hamiltonian Floer homology I: Definition of the Floer homology groups, Trans. Am. Math. Soc. 368, 2967 (2016). 